\title{
Biological effects in human erythrocytes in vitro exposed to xenobiotics : influences by metabolizing systems from rat liver
}

Citation for published version (APA):

Palmen, N. G. M. (1995). Biological effects in human erythrocytes in vitro exposed to xenobiotics : influences by metabolizing systems from rat liver. [Doctoral Thesis, Maastricht University]. Datawyse / Universitaire Pers Maastricht. https://doi.org/10.26481/dis.19950421np

Document status and date:

Published: 01/01/1995

DOI:

10.26481/dis.19950421np

Document Version:

Publisher's PDF, also known as Version of record

Please check the document version of this publication:

- A submitted manuscript is the version of the article upon submission and before peer-review. There can be important differences between the submitted version and the official published version of record.

People interested in the research are advised to contact the author for the final version of the publication, or visit the DOI to the publisher's website.

- The final author version and the galley proof are versions of the publication after peer review.

- The final published version features the final layout of the paper including the volume, issue and page numbers.

Link to publication

\footnotetext{
General rights rights.

- You may freely distribute the URL identifying the publication in the public portal. please follow below link for the End User Agreement:

www.umlib.nl/taverne-license

Take down policy

If you believe that this document breaches copyright please contact us at:

repository@maastrichtuniversity.nl

providing details and we will investigate your claim.
}

Copyright and moral rights for the publications made accessible in the public portal are retained by the authors and/or other copyright owners and it is a condition of accessing publications that users recognise and abide by the legal requirements associated with these

- Users may download and print one copy of any publication from the public portal for the purpose of private study or research.

- You may not further distribute the material or use it for any profit-making activity or commercial gain

If the publication is distributed under the terms of Article $25 \mathrm{fa}$ of the Dutch Copyright Act, indicated by the "Taverne" license above, 
Biological effects in human erythrocytes in vitro exposed to xenobiotics 
Palmen, Nicole Gertrudis Maria

Biological effects in luman erythrocytes in vitro exposed to xenobiotics ; infuences by metabolizing systems from rat liver / Nicole Gertrudis Maria Palmen. - Maastricht. Universitaire Pers Mastricht. - III.

Thesis Rijksuniversiteit Limburg Maastricht. - With ref. With summary in Dutch.

ISBN $90-5278-175-3$

Subject headings: human erythrocytes / xenobiotics / toxicology.

(C) N.G.M. Palmen, Maastricht 1995.

Cover photography:

(c) Boehringer Ingelheim Photography Lennart Nilsson

The printing of this thesis was financially supported by: Stichting Dr. ir. J.H.J. van de Laar 


\title{
Biological effects in human erythrocytes in vitro exposed to xenobiotics
}

Influences by metabolizing systems from rat liver

\author{
PROEFSCHRIFT
}

ter verkrijging van de graad van doctor aan de Rijksuniversiteit Limburg te Maastricht, op gezag van de Rector Magnificus, Prof. mr. M.J. Cohen, volgens het besluit van het College van Dekanen, in het openbaar te verdedigen op vrijdag 21 april 1995 om 14.00 uur

door

Nicole Gertrudis Maria Palmen 


\section{Promotores:}

Prof. dr. P.Th. Henderson (TNO, Rijswijk)

Prof. dr. H.A.J. Struijker Boudier

\section{Co-promotor:}

Dr. ir. C.T.A. Evelo

Beoordelingscommissie:

Prof. dr. M.P. wan Dieijen-Visser (voorzitter)

Prof. dr. J.W. Arends

Dr. B. Blaauboer (Universiteit Utrecht)

Dr. P.J.A. Borm

Prof. dr. V. Rogiers (Vrije Universiteit Brussel, België) 
"Facts do not cease to exist because they are ignored" (A. Huxley)

Aan mijn ouders 



\section{Contents}

1 General introduction

2 Glutathione depletion in human erythrocytes as an indicator for microsomal activation of cyclophosphamide and 3-hydroxyacetanilide

3 Glutathione depletion in human erythrocytes and rat liver; a study on the interplay between bio-activation and inactivation functions of liver and blood

4 Toxicokinetics of dimethylacetamide (DMAc) in rat isolated perfused liver

5 Influence of oxygen supply on liver condition and elimination of dimethylacetamide in the isolated perfused rat liver

6 Oxidlative effects in human erythrocytes caused by some oximes and hydroxylamine

7 General discussion

8 Summary

9 Samenvatting

\section{APPENDICES}

A Dankwoord

B Curriculum vitae

C Lijst van Publicaties 



\section{Chapter 1}

\section{General introduction}

\section{Introduction}

Human beings are exposed to a great variety of compounds of foreign origin (xenobiotics), that are either of natural origin or synthetized by man and are present in food, the environment or more specifically in the work environment [60]. To estimate the nature and extent of health risks imposed by xenobiotics, toxicologists usually apply series of test systems that are mainly based on (or derived from) animal tissues. For the usual purpose - estimation of human risk- data obtained from in vitro and in vivo animal studies have to be extrapolated to man. The uncertainties caused by in vitro to in vivo extrapolation or from the extrapolation from animal to man are taken into account by the introduction of safety factors. Consequently, there is a need for methods or test systems that approach the human in vivo situation as closely as possible. Test systems based on human tissue may be a valuable tool to improve the validity of the extrapolation to man. Moreover, they can give insight into mechanistic aspects of adverse effects in man [11].

Human blood is a biological material which is relatively readily available. It is composed of plasma and cells. Elements in plasma and/or cells may be affected as a result of exposure to xenobiotics. Since blood comes in close contact with organs during its circulation through the arteries and veins, metabolites generated and excreted by the organs may also affect plasma and/or cells. In vitro incubations using human erythrocytes may be a valuable tool in toxicological research since biological effects in erythrocytes caused by xenobiotics can casily be evaluated. The advantages of in vitro incubations over in vivo toxicological research are (i) compounds which cannot be tested in wivo because of ethical reasons can be tested (ii) higher concentrations of the test compound can be tested (iii) the effect of a compound on erythrocytes can be studied without interference of kinetics and/or metabolic activity of other tissues and/or organs. In addition to the direct action of foreign compounds on erythrocytes, xenobiotics may also be metabolised by erythrocytes since these cells also contain metabolic activity themselves $[8,23,41,66,78,80,109]$. Erythrocytes may also be affected by products 
of metabolic activity of other cells. In vitro co-incubation of erythrocytes and other cells can be used in toxicological research to study the interaction between erythrocytes and the other cells. Since the liver is the organ with the highest biotransformational activity, methods were developed in which erythrocytes are co-incubated with liver derived tissue.

Erythrocytes contain an interesting amount of biological parameters that may be affected by xenobiotics or their metabolites. An overview of biological parameters in erythrocytes that were found to be affected in man when exposed to different xenobiotics is given in Table 1.1. Since glutathione concentrations can be affected both by electrophilic and oxidative stress, glutathione depletion was the biological effect parameter which was studied most intensively in this thesis. Oxidation of hemoglobin and lipid peroxidation were evaluated because they are a result of oxidative stress. Glutathione S-transferase activity changes can also be considered as a result of oxidative and electrophilic stress. For this reason this parameter was also tested in this thesis.

\section{Human erythrocytes}

\section{General aspects}

Red blood cells transport oxygen from the lungs to the other tissues and carbondioxide in the opposite direction. The largest fraction of the red blood cell pool consists of mature erythrocytes (>99\%). These cells do neither contain a nucleus nor organelles. Their direct predecessors, reticulocytes, do still contain some basophilic material originating from organelles [40]. Erythrocytes circulate in the body about 120 days before being removed, usually by the spleen [40].

Erythrocytes are exposed to oxidative stress, as a consequence of their oxygen carrying function, and to nutrients and metabolic waste products because of the central role blood plays in their transport. Erythrocytes also come in close contact with xenobiotics directly at the porte d'entrées (i.e. the lungs, the gastro intestinal tract and the skin). Moreover, erythrocytes are also in close contact with cells and organs that may transform non-toxic compounds into toxic metabolites (e.g. the liver). The uptake of these compounds into the erythrocyte depends on the lipid-water partition coefficient, which in turn is dependent on the charge characteristics and the molecular dimensions of a compound [113]. Since erythrocytes possess detoxifying capacity for both electrophilic compounds and reactive oxygen species or products generated by these reactive oxygen species (see section Biochemistry), erythrocytes may play an important role in the protection of organs against these stressors.

To keep their cellular integrity, erythrocytes must protect themselves against (i) oxidants, which may peroxidize the lipid bilayer or oxidize proteins and (ii) elec- 
Table 1.1: Examples of biological events that can occur in erythrocytes as a result of in vivo exposure of humans to xenobiotics and several parameters allied to these biological events.

\begin{tabular}{lll}
\hline Biological event & parameter & Source \\
\hline $\begin{array}{l}\text { membrane } \\
\text { deformation }\end{array}$ & $\begin{array}{l}\text { spectrin-hemoglobin } \\
\text { crosslinking }\end{array}$ & oxidative damage $[96]$ \\
$\begin{array}{l}\text { protein adduct } \\
\text { formation }\end{array}$ & hemoglobin adducts & $\begin{array}{l}\text { aniline }[65,77] \\
\text { ethylene oxide }[17,30] \\
\text { propylene oxide }[79] \\
\text { cigarette smoke }[108,101]\end{array}$
\end{tabular}

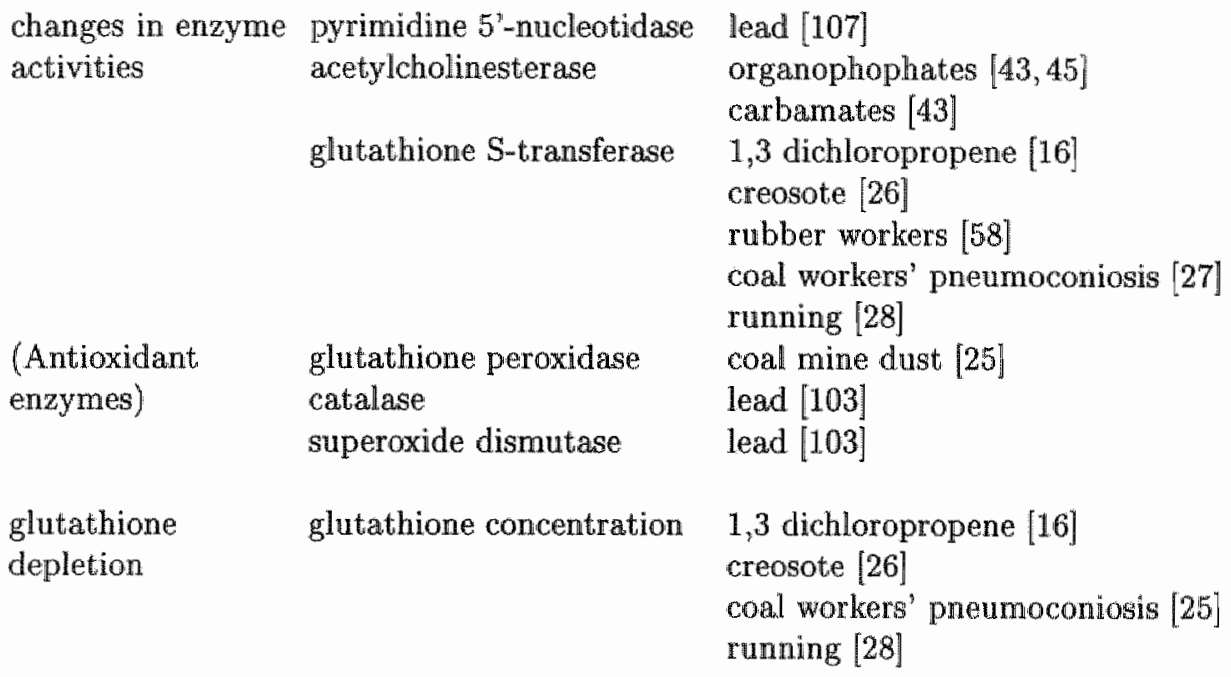

trophiles, which may conjugate to thiol-groups of proteins. Erythrocytes contain several, usually enzyme-regulated, protective mechanisms to minimize injurious effects that result from oxidative products and toxic chemicals. Knowledge of biochemical pathways is necessary to understand the design and interpret the results of experiments as presented in this thesis; therefore biochemical aspects of the parameters that are of concern in this thesis will be made in the following paragraphs. 


\section{Biochemistry}

Erythrocytes need energy and reducing equivalents (i.e. suppliers of electrons) to perform detoxifying reactions. As erythrocytes do not contain mitochondria, energy (ATP) and NADH are supplied by glycolysis [102]. NADP' is reduced to NADPH in the hexose monophosphate shunt [102]. Under normal conditions, $11 \%$ of the glucose phosphorylated by hexokinase into glucose 6-phosphate, passes through the hexose monophosphate shunt. During oxidative stress, this can increase to $92 \%[2,76]$.

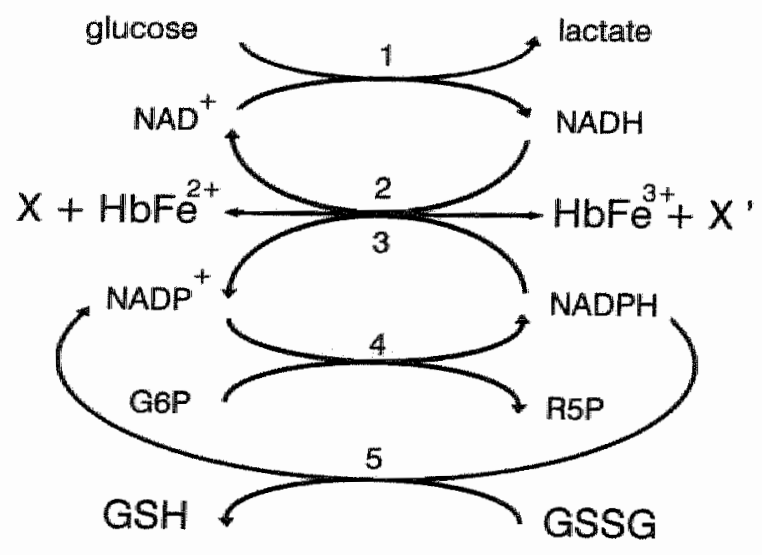

Figure 1.1: Mechanisms to keep erythrocyte hemoglobin in the reduced state, catalyzed by the enzyme(s) 1) of the glycolysis 2) NADH cytochrome bs reductase 3) NADPH flavin reductase 4) of the hexose monophosphate shunt and 5) glutathione reductase. $X$ is a compound that is able to oxidise reduced hemoglobin, with subsequent formation of $X$ ?.

Ferrous hemoglobin can autoxidise into ferric hemoglobin in an environment saturated with oxygen [10]. Since the oxygen carrying function of erythrocytes is confined to ferrous hemoglobin, it must be kept in this reduced state (oxidized hemoglobin $<1 \%$ of total hemoglobin [106]). Erythrocytes possess two enzymes able to reduce oxidized hemoglobin, NADH cytochrome $b_{5}$ reductase and a NADPH flavin reductase (Figure 1.1) $[87,115,116]$. These enzymes use NADH and NADPH respectively to reduce oxidized hemoglobin. NADH cytochrome $b_{5}$ reductase is the most active enzyme, but at impaired cellular integrity, the $\mathrm{Ca}^{2+}$ concentration increases which inhibits NADH cytochrome $b_{5}$ reductase activity and activates NADPH flavin reductase activity [87]. Increased NADPH flavin reductase activity is expected to be of minor importance in vivo since an increase of the $\mathrm{Ca}^{2+}$ concentration in erythrocytes will probably affect the integrity of the cells with subsequent sequestration by the spleen. However, in in vitro incubations in which the oxidation of hemoglobin by a xenobiotic is tested, the 
use of impaired erythrocytes may lead to an increase in NADPH flavin reductase activity. In this way NADPH flavin reductase reduces the effect of an oxidative compound on the formation of oxidized hemoglobin, that was expected as a result of the inhibition of NADH cytochrome $\mathrm{b}_{5}$ reductase alone.

Reduced glutathione (GSH) protects human erythrocytes against oxidative and/or electrophilic stress. As a consequence, the GSH concentration in erythrocytes can be affected. For example, erythrocyte GSH was shown to be decreased in in vitro incubations with methyl chloride, methyl bromide and methyl iodide [41] and as a consequence of in viwo exposure to dichloropropene [16]. Beside the protective effect of GSH on erythrocytes themselves, other cells may also be protected by erythrocyte GSH. Injury of lungs caused by in vivo exposure of rats to hyperoxic conditions, was diminished after insufflation of erythrocytes into the trachea [4]. It was found that the glutathione redox system in erythrocytes and not superoxide dismutiase or catalase, was able to protect the lungs of these rats [44].

The following section will give an overview of glutathione related biochemical reactions that take place in the erythrocyte after oxidative or electrophilic stress. In that section reference will be made to following paragraphs in which glutathione and glutathione dependent enzymes will be discussed.

\section{Overview of GSH biochemistry in relation to oxidative and electrophilic stress}

The viability of erythrocytes is dependent on the degree of exposure to stressors and the ability of the cell to cope with these stressors. Important stressors that may impede cellular functions of the erythrocyte are oxidative stress and exposure to electrophiles. GSH is a tripeptide containing cysteine which has a reactive thiol group with nucleophilic and reductive potency (see section Glutathione) and it plays an important role in the homeostasis of a cell as it (i) protects cells against oxidative challenge and (ii) detoxifies xenobiotics by conjugation [110]. These. reactions are often catalyzed by GSH dependent enzymes. An overview of GSH dependent reactions that may take place in erythrocytes is given in Figure 1.2.

Depletion of glutathione (GSH or total glutathione (GT), which is the sum of both reduced glutathione (GSH) and oxidized glutathione (GSSG)) was the main biological effect parameter tested in this thesis. GSH can be affected as a result of a chemical reaction between GSH and (i) reactive oxygen species, which leads to oxidation of GSH, or (ii) electrophiles resulting in the generation of GSH conjugates. The latter reaction can also be catalyzed by glutathione S-transferase (GST) activity, in which an electrophile is conjugated to GSH (Figure 1.2). GSH bound in this way is not available to the cell anymore as it concerns a covalent bond. The GSH conjugates are also removed from the erythrocyte (see section Glutathione S-transferase). 


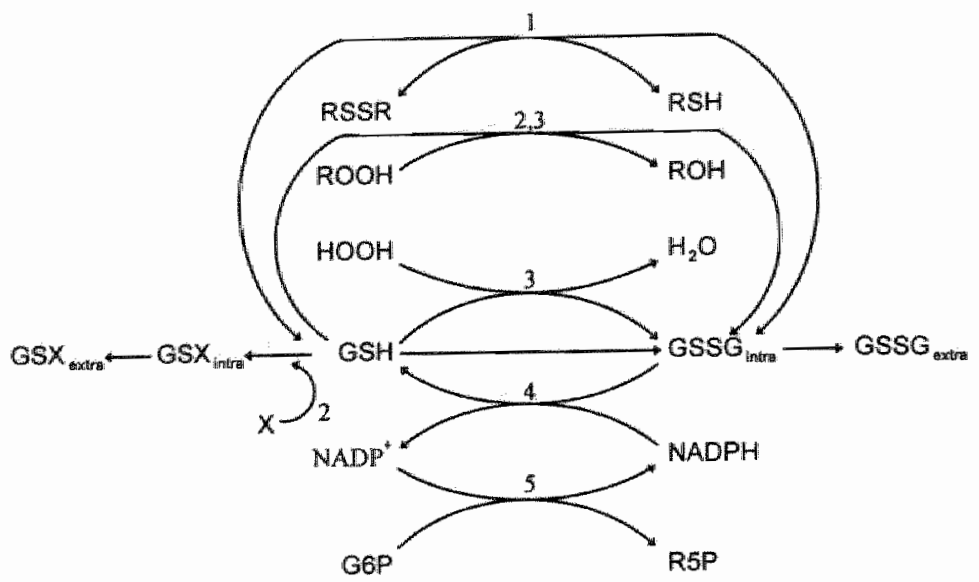

Figure 1.2: Glutathione and glutathione dependent reactions catalyzed by 1) thioltransferase 2) glutathione S-transferase 3) glutathone peroxidase 4) glutathione reductase 5) enzymes of the hexose monophosphate shunt.

Products of oxidative stress, lipid peroxides and disulfides, can be reduced by means of the action of GSH-dependent enzymes with concomitant oxidation of GSH (Figure 1.2). Glutathione peroxidase (GPx) is able to detoxify both $\mathrm{F}_{2} \mathrm{O}_{2}$ and lipid peroxides (see section Glutathione peroxidase). Lipid peroxides can also be reduced by glutathione S-transferase (GST)(see section Glutathione Stransferase); which also uses GSH as reducing equivalent. In contrast to $\mathrm{GPx}$, GST is not able to detoxify hydrogen peroxide. Disulfides between proteins or between proteins and GSH can be reduced by the action of thioltransferase (TT) with concomitant oxidation of GSH (see section Thioltransferase). Since the erythrocyte must keep its reduced state, oxidized glutathione must be reduced. At normal physiological conditions, GSSG is reduced into GSH by glutathione reductase (GR) at the expense of NADPH (see section Glutathione reductase). In the erythrocyte, NADPH is generated in the hexose monophosphate shunt of glycolysis. When the reductive capacity of the erythrocyte (in this case the NADPH/NADP+ ratio) is not high enough, erythrocyte GSSG will be released from the cell (see section Glutathione reductase). Beside release of GSSG from the cell, GSSG can also be stored in the cell by the generation of mixed disulfides of CSH and proteins. This process can also be catalyzed by the action of TT (see section Thioltransferase). In this case, GSSG is stored as a glutathione-protein adduct until the redox potential of the cell becomes normal and $\mathrm{TT}$ is able to liberate GSSG.

In summary, the amount of GSH in the erythrocyte decreases as a consequence of: (a) conjugation with electrophillic compounds, which is often catalyzed by GST 
(b) oxidation of GSH into GSSG by oxidative compounds, (c) peroxidase activity of both GPx and GST and the action of TT with concomitant oxidation of GSH, and (d) the formation of mixed disulfides of GSH and proteins by TT.

\section{Glutathione}

GSH is the most important cellular non-protein thiol. To stabilize cysteine in oxidative surroundings, glutamic acid is linked to cysteine by a $\gamma$-peptide bond [29]. The reaction is catalyzed by the enzyme $\gamma$-glutamylcysteine synthetase $[31,68]$. $\gamma$-Glutamylcysteine cannot be cleaved by normal intracellular peptidases (specific for an $\alpha$-peptide linkage; Figure 1.3) [98]. GSH is formed by the linkage of glycine to $\gamma$-glutamylcysteine, which is catalyzed by glutathione synthetase. Glycine coupling to the dipeptide counteracts oxidation still further [29].

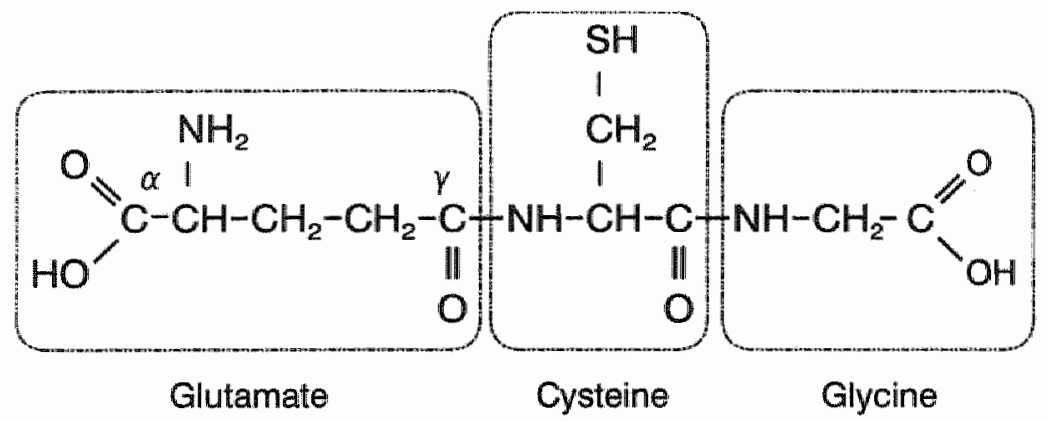

Figure 1.3: glutathione

The erythrocyte has a GT concentration of about $2 \mathrm{mM}$ [31]. For the most part, erythrocyte glutathione is in the reduced state with minor fractions being GSSG. The GT content in erythrocytes is a result of the balance between the rate of synthesis of glutathione and the rate of elimination. Half-life of erythrocyte glutathione is $2-4$ days $[9,99]$. GSH cannot be transported into the erythrocyte over the erythrocyte membrane [114], but is synthetized in erythrocytes by means of the glutathione synthetizing enzymes $\gamma$-glutamylcysteine synthetase and glutathione synthetase $[31,68]$. Glutathione synthesis is regulated by the negative feed-back of glutathione on $\gamma$-glutanylcysteine synthetase [82], and the availability of the three amino acids [9]. Since erythrocytes lack $\gamma$-glutamyltranspeptidase activity $[99,98]$, transport of amino acids over the erythrocyte membrane may be a problem. Especially the availability of glutamate is considered to be essential since the permeability of the erythrocyte membrane for this amino acid is very low [9]. Export of GSSG or GSH-conjugates over the erythrocyte membrane will be discussed in a separate paragraph on page 18. 


\section{Glutathone peroxidase}

As a consequence of oxidative stress, membrane lipids may be peroxidized. One of the products generated during lipid peroxidation (LP) is malondialdehyde, which is often used as an indicator for LP [89]. In this thesis the formation of lipid peroxides was determined by means of thiobarbituric reactive substances (TBARS) [93]. Lipid peroxides may be detoxified by glutathione peroxidases. There are two classes of cytosolic glutathione peroxidases, the selenium dependent GPX [32] and the selenium independent GST (see section Glutathione Stransferase). GPx reduces organic hydroperoxides into lipid alcohols. As a consequence GSH is oxidized into GSSG (Figure 1.2) [32]. The enzyme is highly specific for GSH as a reducing substrate and GSH supply is critical for erythrocyte GPx activity [33]. The GPx level in human erythrocytes is usually high and, together with catalase, plays a role in the detoxification of $\mathrm{H}_{2} \mathrm{O}_{2}$ [32]. GPx activity is sensitive to superoxide anions which can react with the selenolate in the active site of the enzyme [32]. GSSG which is formed during the GPx reaction is reduced by GR at the expense of NADPH. Erythrocytes possess both a hydrophilic and a hydrophobic GPx [32]. As hydrophilic cytoplasmic GPx does not directly reduce hydroperoxo groups in complex lipids [32], cleavage of peroxidized fatty acids by phospholipase must precede the activity of hydrophilic GPx activity $[32,62,104]$. However, (direct) lipophilic GPx activity may be of more importance for the detoxification of lipid membrane hydroperoxides.

\section{Thioltransferase}

Erythrocyte TT (EC 1.8.4.1) belongs to the superfamily of thiol-disulfide oxidoreductases. It has a molecular weight of $11300 \mathrm{D}$ and contains four cysteine residues per protein molecule $[73,74]$. The average level of activity of TT in erythrocytes $\left(4.6 \mathrm{U} \cdot \mathrm{g}^{-1} \mathrm{Hb}\right.$ at $\left.25^{\circ} \mathrm{C}\right)$ is in the same order of magnitude as GR and G6PDH $[29,35,36,70,73]$. TT requires GSH and GR to support its catalytic role in the reduction of oxidized thiol groups in proteins as well as in low molecular mass compounds (see also Figure 1.2). TT along with GR and G6PDH may play a crucial role in erythrocytes sulfhydryl homeastasis by protecting SH-groups on enzymes, hemoglobin and proteins important for membrane integrity. Moreover, at high intracellular GSSG concentrations, TT is also able to store GSSG in the form of mixed disulfides [70].

\section{Glutathione reductase}

GR (E.C. 1.6.4.2.; $M=125000 \mathrm{D})$ is a dimer which binds 1 flavin adenine dinucleotide (FAD) per subunit [102]. It has a binding site for GSSG, NADPH and FAD $[67,70,102]$. Electrons are supplied by NADPH and transported via FAD to the active site $[67,70]$, which is a disulfide [29]. As a result, GSSG is reduced to 
GSH $[29,67,70]$ (Figure 1.2). As GR is inactivated by NADPH by modification of the reduced enzyme, $G R$ activity is strongly decreased at normal physiological conditions [67]. GSSG protects GR against inactivation and is also able to reactivate the enzyme [67]. In case of oxidative stress, the GSSG concentration increases which activates GR to reduce GSSG into GSH, with concomitant increase of the NADP ${ }^{*}$ concentration. The increased NADP ${ }^{+}$concentration overcomes the inhibition of glucose 6-phosphate dehydrogenase of the hexose monophosphate shunt, so that NADP ${ }^{*}$ is reduced into NADPH [67], which can be used for the reduction of GSSG. The very low GSSG/GSH ratio is maintained by GR activity $[9,67]$ and export of GSSG (see further).

\section{Glutathione S-transferase}

GST's (E.C. 2.5.1.18) are enzymes which can be found in the cytosol or in microsomes. As erythrocytes do not have organelles, they only possess cytosolic GST. Human cytosolic GST's are divided in class $\alpha, \mu, \pi$ and $\theta[69,72]$. It depends on the cell type which GST class or classes are expressed [69]. The majority of human erythrocyte GST consists of GST- $\rho$ (90-95\%) which is a GST- $\pi$ class enzyme $[9,12,14,69]$. Erythrocytes also contain GST- $\sigma$, a minor form of erythrocyte GST, which was characterized and differentiated from the major form by Awasthi et al. [6]. More recently, another GST was found which is responsible for the conjugation of methyl halides in erythrocytes $[41,42,86]$. Expression of this $\theta$-class enzyme is known to be polymorfic [41,42]. Erythrocyte GST has a $G$ (glutathione binding) site and a $\mathrm{H}$ (substrate binding) site. The G-site is highly specific for GSH but the H-site has a broad substrate specificity. GSH makes the enzyme catalytically competent by inducing a conformational change as well as providing the functional thiol group for the reaction to be catalyzed [70]. Since the $K_{m}$ for GST binding in erythrocytes is $0.6 \mathrm{mM}[6]$ and the erythrocyte GSH concentration is about $2 \mathrm{mM}$, GST will be saturated with GSH.

The nonsubstrate haem can be transported by GST [12]. An interesting quality of GST is its catalytical action upon the conjugation of GSH with electrophiles via substitution or addition of GSH [18], which is usually a detoxification pathway (Figure 1.2). However, a number of compounds can also be activated in this reaction by the formation of episulfonium ions [19]. In vivo, erythrocyte conjugation of electrophiles to GSH may be of great importance as GST- $\rho$ catalyses the conjugation of other electrophiles than e.g. GST- $\alpha$ of the liver [31]. GST- $\rho$ is also abie to reduce organic hydroperoxides using GSH as reducing equivalent, with subsequent release of GSSG and an alcohol (Figure 1.2) $[12,22,29,75]$, which is also called the selenium-independent peroxidase activity of GST [56]. The peroxidase activity is restricted to organic hydroperoxides. Unlike glutathione peroxidase (see Glutathione peroxidase), GST is not able to detoxify $\mathrm{H}_{2} \mathrm{O}_{2}$. The peroxidase activity of GST consists of two reactions, the first one is catallyzed by GSI, the second one is a non-enzymatic reaction which requires another GSH molecule [32]. 


$$
\begin{aligned}
& \mathrm{ROOH}+\mathrm{GSH} \stackrel{\mathrm{GST}}{\longrightarrow} \mathrm{ROSG}+\mathrm{H}_{2} \mathrm{O} \\
& R O S G+G S H \longrightarrow R O H+G S S G
\end{aligned}
$$

As the organic hydroperoxide must be released from the cellular membrane before it can be reduced by cytosolic GST, the action of phospholipase is a requirement $[56,57,62,104]$. This was also the case in GPx-mediated reduction of organic hydroperoxides.

\section{Export of glutathione and glutathione conjugates}

Both GSSG and conjugates of GSH and electrophiles (glutathione S-conjugates) can be removed from the erythrocyte by means of active transport [9]. GSSG can be exported by two different ATP-ases, one with a high affinity for GSSG $\left(K_{\mathrm{m}}=0.1 \mathrm{mM}\right)$ and one with a low GSSG affinity $\left(K_{\mathrm{m}}=7.1 \mathrm{mM}\right)[9]$. At low intracellular GSSG concentrations, GSSG will mainly be transported by the high affinity carrier. However, its capacity is limited as the $V_{\max }$ is $20 \mathrm{nmoles} \cdot \mathrm{ml}^{-1} \mathrm{~h}^{-1}[61]$. The $V_{\max }$ of the low affinity carrier is ten times higher, so that GSSG export. by this carrier may be important in case of oxidative stress. The low affinity carrier is also able to transport the S-(2,4 dinitrophenyl)-glutathione. The $K_{\mathrm{m}}$ values for the glutathione S-conjugate and ATP are $0.29 \mathrm{mM}$ and $1.0 \mathrm{mM}$ respectively $[7,64]$. Awasthi et al. demonstrated that the transport of glutathione S-conjugates is probably the most important function of the low affinity carrier in vivo, since GSSG was not transported anymore in the presence of $S-(2,4$ dinitrophenyl)-glutathione even though the transport of S-(2,4 dinitrophenyl)glutathione was not inhibited by GSSG concentrations up to $11.8 \mathrm{mM}$ [7]. This means that glutathione S-conjugates limit the export of GSSG by the low affinity carrier. Since the high affinity carrier was not affected by the glutathione S-conjwgate, it is likely that this carrier serves to protect the erythrocyte from the toxic intracellular effects of GSSG [9]. Since S-(2,4 dinitrophenyl)-glutathione also inhibits GR activity [92], glutathione S-conjugates may have an unfavorable effect on the GSH/GSSG redox status of the cell, firstly by the consumption of GSH required for conjugate formation, secondly by the inhibition of GSSG transport from the cell by the conjugate, and thirdly by inhibition of GR. Glutathione Sconjugates may also be able to affect GST activity, which was reported for e.g. S-(2-chloro 4-nitrophenyl)-glutathione [92].

\section{The rat liver}

As discussed above, xenobiotics may affect biochemical reactions and targets in erythrocytes. Various organs can transform a non-reactive parent compound 
into metabolites that may affect erythrocytes. As the liwer is the organ with the highest metabolic capacity, the effect of the addition of rat liver metabolizing systems to in vitro incubations with erythrocytes is investigated in this thesis. For this reason, several biochemical aspects of the rat liver will be discussed.

The rat liver plays an important role in the metabolism of carbohydrates, fat and proteins, and in the biotransformation of xenobiotics. It is mainly composed of hepatocytes $(60-65 \%$ of the total cell number [37] and $78 \%$ of the liver volume [13]), which are responsible for a large fraction of the metabolic activity of the liver. A cell layer of hepatocytes is arranged along the sinusoids that spans the portovenous distance. Afferent vessels are the hepatic arterioles and portal venules and efferent vessels are the central vein and the bile ducts [40]. The high oxygen consumption of the liver leads to a steep gradient along the sinusoids as well as from the center of the sinusoid to the plasma membranes of the cells and intracellularly to the mitochondria [37]. Structural and functional differences between rat hepatocytes from different locations have been observed, especially regarding enzyme activities (see below) [49].

Biotransformation of xenobiotics by the liver can be divided in phase I and phase II activity. Phase I activity comprises oxidation, reduction and hydrolysis of xenobiotics and phase II activity includes conjugation or synthetic reactions $[3,60]$. Although phase I and II metabolism are regarded as detoxifying reactions, some chemicals may also be converted into more toxic metabolites [39].

Phase I reactions add functional groups (e.g. $-\mathrm{OH},-\mathrm{SH},-\mathrm{NH}_{2},-\mathrm{COOH}$ ) to the parent compound. Enzymes of the cytochrome P-450 gene superfamily are considered the most important phase I enzymes [60]. Cytochrome P-450 catalyzes the mono-oxygenation of a substrate in the presence of oxygen and reducing equivalents [105]. In man, minor changes in the oxygen supply to the liver may depress the cytochrome P-450 activity as the $K_{\mathrm{mo}}$ of cytochrome $\mathrm{P}-450(10-15 \mu \mathrm{M})$ is in the same range as the mean in wa intra-hepatic oxygen concentration (about $35 \mu \mathrm{M})[3]$. The cytochrome P-450 enzymes are embedded in the smooth endoplasmic reticulum [105], and as such are a constituent of microsomal preparations. The total cellular cytochrome P-450 content increases from the periportal to the perivenous site of the liver lobule $[38,47,88,95,100,112]$. Since the oxygen concentration decreases in that direction, the $\mathrm{O}_{2}$ supply for cytochrome $\mathrm{P}-450$ is even more critical than would be expected from the mean intrahepatic $\mathrm{O}_{2}$ concentration. Treatment of animals with phenobarbitone leads to an increase in the perivenous cytochrome P-450 content and to an increase in the proliferation of the endoplasmic reticulurn $[46,47]$.

By means of phase II metabolism, functional groups on a xenobiotic may be compled to endogenous substrates. The functional groups may be produced by phase I metabolism, but there are also compounds that can immediately undergo conjugation reactions [3]. In this way, reactive componunds may be detoxified in 
the hepatocyte so that other cells (including erythrocytes) will not be exposed to these reactive compounds, as would be the case if they would be able to leave the hepatocyte unchanged. A great number of phase II reactions are known to occur in the liver, but only the reactions that are of importance for the understanding of the material presented in this thesis will be described.

- Glucuronidation is one of the most important phase II reactions. In this reaction, uridine diphosphate glucuronic acid (UDP-glucuronic acid) is coupled to the functional group on the acceptor molecule. The reaction is catalyzed by UDP-glucuronosyl transferase which has a very broad substrate specificity [60]. UDP-glucuronosyltransferase activity is higher in perivenous hepatocytes $[3,24]$. Glucuronide conjugates may be deconjugated by the hydrolase $\beta$-glucuronidase, which seems to be evenly distributed across the liver lobule $[24,50]$.

- Sulfation is an important conjugation reaction for hydroxyl groups. The reaction is catalyzed by sulfotransferases, which transfer activated sulfate to the hydroxyl group present on phenols and aliphatic alcohols [60]. Sulfotransferase activity was higher in periportal than in perivenous hepatocytes when umbelliferone was tested $[3,24]$. Aryl sulfatases may degrade aryl-sulfate conjugates of xenobiotics. They seem to be evenly distributed over the liver lobule $[24,50]$.

- Glutathione S-transferases catalyze the conjugation of electrophiles with the nucleophilic sulfhydryl of GSH [18]. The hepatic GT concentration is depleted in this way. Hepatocytes possess both cytosolic GST's and GST's associated with the endoplasmic reticulum [75]. There is a large structural difference between the two types of GST's as cytosolic GST is a dimer [57,69] and endoplasmic reticulum bound GST has three subunits [75]. The main cytosolic GST class found in hepatocytes is GST- $\alpha$ [69]. Hepatic cytosolic GST activity which is responsible for conjugation of GSH to electrophiles, is found predominantly in isolated perivenous rat hepatocytes $[54,55]$.

Partly due to the action of GST, hepatocyte GSH can be depleted. Beside GSH conjugation, the hepatic glutathione pool can also be depleted by transport of GSSG over the hepatocyte membrane. This occurs when the reductive capacity of the hepatocyte is not sufficient to maintain the low GSSG/GSH ratio [1]. GSSG is formed in the peroxidase reaction of GST in which peroxidized lipids are reduced into alcohols [32]. Unlike the transferase activity, the peroxidase activity of GST was found to be higher in the periportal area [54]. This may be explained by the distribution of GST isoenzymes over the lobulus. GPx is able to reduce both $\mathrm{H}_{2} \mathrm{O}_{2}$ and peroxidized lipids with concomitant GSSG formation [32]. In accordance with the peroxidase activity of GST, GPx activity was also found to be higher in periportal hepatocytes [54]. GSH is also used as reducing equivalent by thioltransferase in the reduction of protein disulfides or mixed disulfides of glutathione and protein, though its activity in rat liver is low [35]. Beside conjugation 
of glutathione with electrophiles, cytosolic GST's are involved in the formation of $\Delta^{5} 3$-ketosteroids and prostaglandines [12]. They also bind the nonsubstrates bilirubin and other bile acids [12].

The synthesis of glutathione by the liver is $0.5 \mathrm{nmoles} \cdot \mathrm{mg}$ protein ${ }^{-1} \cdot \mathrm{min}^{-1}$ [89], and is normally higher than the glutathione consumption. Under normal conditions liver GSH is released into plasma and bile and used by other organs. Neither GSH nor GSSG is taken up by the liver [1], as hepatocytes do not have $\gamma$-glutamyltranspeptidase activity $[37,59]$. According to Kera et al., Shimizu and Morita, Smith et al. and Väänäen [53,90,94,111] periportal hepatocytes contain more glutathione. "This in contrast with Asghar et al., Deml and Oesterle, Schön and Speisky et al. $[5,20,85,97]$, who found a more evenly distributed glutathione concentration across the lobule. Intracellularly, glutathione is found both in the cytosol (90\% of the GT concentration) and in mitochondria (10\% of the GT concentration) $[81,83,110]$. Mitochondria lack the ability to synthetize GSH [83] and are unable to export GSSG [110]. Depletion of GSH by electrophiles or oxidation into GSSG by oxidative compounds is compatible with life as long as the mitochondrial GSH pool is not depleted too much. Both cytosolic GSSG and GSH adducts (e.g. S-(2,4 dinitrophenyl)-glutathione) are exported from the hepatocytes into bile $[1,48,91,92]$. There is even a mutual competition between transport of GSSG and GSH-conjugates [91,92]. In this way, GSH conjugates may have an unfavorable effect on the GSH/GSSG redox status in the cell, firstly by the consumption of GSH required for conjugate formation, and secondly by the inhibition of GSSG transport from the cell by the conjugate which was also described for erythrocytes $[91,92]$.

\section{Objectives and outline of the thesis}

Exposure of living organisms to high doses of xenobiotics may lead to adverse effects. Chronic exposure of living organisms to low doses of xenobiotics may also lead to impairment of health [26]. At an earlier stage in time, biological parameters at the cellular level may be affected [26]. In the preceding text it was demonstrated that human erythrocytes possess an interesting amount of targets and biochemical reactions to study the effect of xenobiotics. An other advantage of human erythrocytes is that they are readily available.

Since in wivo there is a close contact between blood and hepatocytes, non-reactive xenobiotic compounds may be metabolised by the liver into compounds that cause biological effects in erythrocytes. In this thesis main attention is directed to the interplay between erythrocytes and hepatocytes with special emphasis to the bioactivation and inactivation capacity of liver and blood. To enable such studies, several in vitro techniques combining erythrocytes and rat liver metabolizing systems had to be developed and evaluated, as is illustrated in Figure 1.4. 
Liver

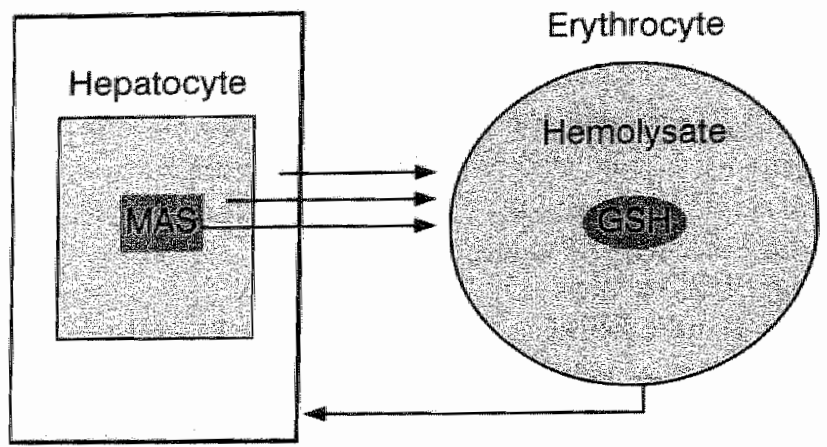

Figure 1.4: Diagram that outlines the incubations performed in this thesis. Glutathione solution (GSH), hemalysate and erythrocytes were used as GSHcontaining target systems. As compounds may have to be metabolised to deplete GSH, metabolically activating systems were also added to the incubations. The metabolically activating systems used in this thesis consisted of microsomal activating system (MAS), hepatocytes and total liver. Finally, the ability of erythrocytes to affect the viability and biotransformational capacity of the total liver was evaluated $(\rightarrow)$.

Several compounds with known effects were used as positive controls to develop and understand the test systems. Compounds tested in this thesis can be divided into (i) compounds with known electrophilic capacity (iodoacetamide, $\mathbb{N}$ ethylmaleimide and diethyl maleate), (ii) a strong oxidator (hydroxylamine) and (iii) compounds that have to be metabolised to be able to deplete GT (cyclophosphamide, 3-hydroxyacetanilide, 2-methylfurane). Subsequently, the test systems were applied to several test compounds with unknown effects. Two pesticides, monuron and monulinuron, were tested since their metabolite 4-chloroaniline is able to cause hemoglobin adducts in rats [84]. Because the adducts are attached to the thiol-group of hernoglobin, other thiols may also be affected. The industrial chemicals cyclohexanone oxime, acetaldoxime and methyl-ethyl ketoxime were tested since oximes are known hematotoxic compounds in rats. Among others, cyclohexanone oxime causes hemoglobin oxidation and an increase in circulating reticulocytes $[21,34]$ and methyl-ethyl ketoxime causes a decrease in erythrocyte count and hemoglobin content [63]. Workers exposed to dimethylacetamide (DMAc) are able to excrete the metabolite monomethylacetamide $[71,15,52]$. The metabolism of DMAc is believed to be confined to the liver [51]. In our studies DMAc is used to test whether an isolated rat liver is able to metabolize this compound. Purthermore, DMAc is also used to test whether erythrocytes affect DMAc elimination kinetics in an isolated perfused rat liver. 
The outline of the thesis is as follows:

- Microsomal preparations were used in chapter 2 as metabolic activating system. GT depletion by active compounds was studied as parameter of toxicity.

- The metabolic capacity of microsomal activating system and intact hepatocytes was compared in chapter 3. Depletion of GT or GSH was used as toxicological parameter.

- In chapter 4 the biotransformational capacity of an isolated totol liver was studied by means of the investigation of the elimination kinetics of DMAC. Thereupon chapter 5 describes the influence on DMAc elimination kinetics by addition of erythrocytes to the liver perfusion system.

- Chapter 6 deals with a comparative study. It describes whether the different combinations with either microsomal preparations or hepatocytes or isolated total liver and erythrocytes are useful to establish the toxicological profile of hydroxylamine and several oximes. Beside GT, several other endpoints have been measured.

- In chapter 7 the results in the studies performed in this thesis are discussed, and they are summarised in chapter 8. 


\section{Bibliography}

[1] Akerboom, T. and Sies, H. Glutathione transport and its significance in oxidative gtress, in: Vina, I. (ed.) Glutathione: metabolism and physiological functions; CRC Press, Boca Raton, 1990, 46-55.

(2] Albrecht, V., Roigas, H., Schultze, M., Jacobasch, G. and Rapoport, S. (1971) The influence of $\mathrm{pH}$ and methylene bleu on the pathways of glucose utilization and lactate formation in erythrocytes of man. Eur. J. Biochem. 20, 44-50.

[3] Angus, P.W., Morgan; D.J. and Smallwood R.A. (1990) Review article: hypoxia and hepatic drug metabolism-clinical implications. Aliment. Pharmacol. Therap. $4,213-225$.

(4] Asbeck van, S., Hoidal, J, Schwartz, B., Vercellotti, G.M., Moldow, C.F. and Jacob, H.S. (1984) Insufflated red cells protect lungs from hyperoxic damage: role of red cell glutathione in scavenging toxic $O_{2}$ radicals. Transact. Ass. Am. Physicians 97, 365-368.

[5] Asghar, K., Reddy, B.G. and Krishna, G. (1975) Histochemical localization of ghtutathone in tissues. J. Histochem. Cytochem. 23, 7774-7779.

(6) A wasthi, Y.C. and Singh, S.V. (1984) Purification and characterization of a new form of glutathione S-transferase from human erythrocytes. Biochem. Biophys. Res. Commum. 125, 1053-1060.

[7] A wasthi, Y.C., Singh, S.V., Ahmad, L.W., Wronski, S.K, Srivastava, S.K. and LaBelle, E.F. (1989) ATP dependent primary active transport of xenobioticglutathione conjugates by human erythrocyte membrane. Mol. Cell. Biochem. $91,131-136$.

[8] Belvedere, G. and Tursi, F. (1981) Styrene oxidation to styrene oxide in human blood erythrocytes and lymphocytes. Res. Commun. Chem. Pathol. Pharmacol. $33,273-282$.

[9] Beutler, E. and Dale, G.L. Erythrocyte glutathione: function and metabolism, in: Dolphin, D., Avramovic, O and Poulson, R. (eds.) Glutathione; chemical, biochemical, and medical aspects, J. Wiley and sons, New York, part B, 1989, pp. $291-317$.

[10] Blauboer, B.J. and Woutersen, R.A. (1988) Toxicologie van bloed en bloedvormende organen, Pudoc, Wageningen, 1988, p. 35.

[11] Blauboer, B.J. The significance and applicability of human tissues and cells as in vitro models in toxicology, in: Rogiers, V., Sonck, W., Shephard, E. and Vercruysse, A. (eds.) Human cells in in vitro pharmaco-toxicology, VUB Press, Brussels, 1993, pp. 1-9.

[12] Bladeren, P.J. and Ommen, B. (1991) The inhibition of glutathione S-transferases: mechanisms, toxic consequences and therapeutic benefits. Pharmac. Ther. $51,35-46$.

[13] Blouin, A., Bolender, R.P. and Weibel, E.R. (1977) Distribution of organelles and membranes between hepatocytes and nonhepatocytes in the rat liver parenchyma. A stereological study. $J$. Cell Biol. $72,441-455$.

[14] Board, P., Coggan, M., Johnstom, P., Ross, V., Suzuki, T. and Webb, G. (1990) Genetic heterogeneity of the human glutathione transferases: a complex of gene families. Pharmac. Ther. 48, 357-369. 
[15] Borm, P.J.A., DeJong, L. and Vliegen, A. (1987) Environmental and biological monitoring workers occupationally exposed to dimethylacetamide. J. of Occup. Med. 29, 898-903.

[16] Brouwer, E.J., Evelo, C.T.A., Verplanke, A.J.W., van Welie, R.T.H. and de Wolff, F.A. (1991) Biological effect monitoring of occupational exposure to 1,3dichloropropene: effects on liver and renal function and on glutathione conjugation. Br. J. Indus. Med. 48, 167-172.

[17] Calleman, C.J., Ehrenberg, L. Jansson, B., Osterman-Golkar, S., Segerback, D., Svensson, K. and Wachtmeister, C.A. (1978) Monitoring and risk assessment by means of alkyl groups in hemoglobin in persons occupationally exposed to ethylene oxide. J. Environ. Pathol. Toxicol. 2, 427-442.

[18] Chasseaud, L.F. Conjugation with glutathione and mercapturic acid excretion, in: Arias I.M. and Jakoby, W.B. (eds.) Glutathione: metabolism and function, Raven Press, New York, 1976, pp. 77-114.

[19] Coles, B. and Ketterer, B. (1990) The role of glutathione and glutathione Stransferases in chemical carcinogenesis. Crit. Rev. Biochem. Mol. Biol. 25, 47-66.

[20] Deml, E. and Oesterle, D. (1980) Histochemical demonstration of enhanced glutathione content in enzyme altered islands induced by carcinogens in rat liver. Cancer Res. 40, 490-491.

[21] Derelanko, M.J., Gad, S.C., Powers, W.J., Mulder, S., Gavigan, F. and Babich, P.C. (1985) Toxicity of cyclohexanone oxime; I. Hematotoxicity following subacute exposure in rats. Fundam. Appl. Toxicol. 5, 117-127.

[22] Douglas, K.T. Reactivity of glutathione in model systems for glutathione Stransferases and related enzymes, in: Sies, H. and Ketterer, B. (eds.) Glutathione conjugation; mechanisms and biological significance, Academic Press, London, 1988, pp. 2-41.

[23] Duverger-van Bogaert, M., Crutzen-Fayt, M.C. and Stecca, C. (1991) Mutagenicity of some heterocyclic amines in Salmonella typhimurium with metabolic activation by human red blood cell cytosol. Mutat. Res. 261, 261-265.

[24] Elmouelhi, M. and Kauffman, F.C. (1986) Sublobular distribution of transferases and hydrolases associated with glucuronide, sulfate and glutathione conjugation in human liver. Hepatology 6, 450-456.

[25] Engelen, J.J.M., Borm, P.J.A., van Sprundel, M. and Leenaerts, L. (1990) Blood anti-oxidant parameters at different stages of pneumoconiosis in coal workers. Environ. Health Persp. 84, 165-172

[26] Evelo, C.T.A. and Henderson P.Th. (1991) Biological effect monitoring. Arch. Toxicol. Suppl. 15, 268-277.

[27] Evelo, C.T.A., Bos, P.B. and Borm, P.J.A. (1993) Decreased glutathione content and glutathione S-transferase activity in red blood cells of coal miners with early stages of pneumoconiosis. Br. J. Ind. Med. 50, 633-636.

[28] Evelo, C.T.A., Palmen, N.G.M., Artur, Y. and Janssen, G.M.E. (1992) Changes in blood glutathione concentrations, and in erythrocyte glutathione reductase and glutathione $S$-transferase activity after running training and after participation to contests. Eur. J. Appl. Physiol. 64, 354-358. 
[29] Fahey, R.C. and Sundquist, A.R. (1991) Evolution of glutathione metabolism. Adv. Enzymol. Rel. Areas Molec. Biol. 64, 1-53.

[30] Farmer, P.B., Bailey, E., Gorf, S.M., Törnquist, M., Osterman-Golkar, S., Kautlainen, A. and Lewis-Enright, D.P. (1986) Monitoring human exposure to ethylene oxide by the determination of havmoglobin adducts using gas chromatography-mass spectrometry. Carcinogenesis 7, 637-640

[31] Fazi, A., Mancini, U., Piatti, E., Accorsi, A. and Magnani, M. (1991) Human red blood cells as bioreactors for the inactivation of harmful xenobiotics. Biotechnol. Appl. Biochem. 14,60-68.

[32] Flohé, L. The selenoprotein glutathione peroxidase, in: Dolphin, D. Poulson, R. and Avramovic, O. (eds.) Glutathione; chemical, biochemical, and medical aspects, $J$. Wiley and Sons, New York, part A, 1989, pp. 643-731.

[33] Flohe, L. Structure and catalytic mechanism of glutathione peroxidase, in: Taniguchi, N., Higashi, T., Sakamoto, Y. and Meister, A. (eds.) Glutathione centennial: moleculare perspectives and clinical implications, Academic Press, San Diego, 1989, pp. 103-114.

[34] Gad, S.C., Derelanko, M. I., Powers, W.J., Mulder, S., Gavigan, F. and Babich, P.C. (1985) Toxicity of cyclohexanone oxime: II. Acute dermall and subchronic oral studies. Fundam. Appl. Toxicol. 5, 128-136.

[35] Gan, Z. and Wells, W.W. (1986) Purification and properties of thioltransferase. J. Biol. Chem. 261, 996-1001.

[36] Gan, Z. and Wells, W.W. (1987) Preparation of homogeneous pig liver thioltransferase by a thiol: disulfide mediated pI shift. Anal. Biochem. 162, 265-273.

[37] Gebhardt, R. (1992) Metabolic zonation of the liver: regulation and implications for liver function. Pharmac. Ther. 53, 275-354.

[38] Gooding, P.E., Chayen, J., Sawyer, B. and Slater, T.F. (1978) Cytochrome P-450 distribution in rat liver and the effect of sodium phenobarbitone adminitration. Chem. Biol. Interact. 20, 299-310.

[39] Grant, D.M. (1991) Detoxification pathways in the liver. J. Inher. Metab. Dis. 14, $421-430$.

[40] Guyton, A.C. Textbook of medical physiology, W.B. Saunders company, Philadelphia, 1986, pp. 41-50.

[41] Hallier, E., Deutschmann, S. Reichel, C., Bolt, H.M. and Peter, H. (1990) A comparative investigation of the metabolism of methyl bromide and methyl iodide in human erythrocytes. Int. Arch. Occup. Health 62, 221-225.

[42] Hallier, E, Langhof, T., Dannappel, D., Leutbecher, M., Schröder, K., Goergens, H.W., Müller, A. and Bolt, H.M. (1993) Arch. Toxicol. 67, 173-178.

[43] He, F. (1993) Biological monitoring of occupational pesticides exposure. Int. Arch. Occup. Environ. Health 65, S69-S76.

[44] Hoidal, J.R., Van Asbeck, B.S., Mann, J., Jacob, H.S. and Kennedy T.P. (1988) Therapy with red blood cells decreases hyperoxic pulmonary injury. Exp. Lung
Res. 14,977 -985 .

[45] Jauhiainen, A., Kangas, J., Laitinen, S. and Savolaimen, K. (1992) Biological monitoring of workers exposed to Mevinphos in greenhouses. Bull. Environ. Contam. Toxicol $49,37-43$. 
[46] Kanai, K. Wanatabe, J. and Kanamura, S. (1986) Quantitative analysis of smooth and rough endoplasmic reticulum proliferation in differentiating hepatocytes of midpostnatal mice treated with phenobarbital. J. Ultrastruct. Molec. Struct. Res. 97, 64-72.

[47] Kanai, K., Wanatabe, J. and Kanamura, S. (1990) Microphotometric analysis of cytochrome P-450 in periportal, midzonal and perivenular hepatocytes of mice treatied with phenobarbital. J. Histochem. Cytochem. 38, 1599-1605.

[48] Kaplowitz, N. Hepatic glutathione transport, in: Taniguchi, N., Higashi, T.。 Sakamoto, Y. and Meister, A. (eds.) Glutathione centennial: moleculare perspectives and clinical implications, Academic Press, San Diego, 1989, pp. 395-406.

[49] Katz, N.R. (1989) Methods for the study of liver cell heterogeneity. Histoch. J. $21,517-529$.

[50] Kaufman, F.C. and Matschinsky, F. Quantitative histochemical measurements within sublobular zones of the liver lobule, in: Thurman, R.G., Kauffiman, F.C. and Jungermann, $K$. (eds.) Regulation of hepatic metabolism: intra- and intercellular compartmentation, Plenum, New York, 1986, pp. 119-136.

[51] Kennedy, G.L. (1986) Biological effects of acetamide, formamide and their monomethyl and dimethyl derivatives. CRC Crit. Rev. Toxicol. 17, 129-182.

[52] Kennedy, G.L and Pruett, J.W. (1989) Biologic monitoring for dimethylacetamide: measurement for 4 consecutive weeks in a workplace. J. Occup. Med. 31 , $47-50$.

[53] Kera, Y., Penttila, K.E. and Lindros, K.O. (1988) Glutathione replenishment capacity is lower in isolated perivenous than in periportal hepatocytes. Biochem. J. $254,411-417$.

[54] Kera, Y., Sippel, H.W., Penttila, K.E. and Lindros, K.O. (1987) Acinar distribution of glutathione-dependent detoxifying enzymes. Low glutathione peroxidase activity in perivenous hepatocytes. Biochem. Parmacol. 36, 2003-2006.

[55] Kera, Y., Speisky, H., Penttilla, K.E., Sippel, H. and Lindros, K.O. (1987) Glutathione metabolism in isolated rat hepatocytes: acinar heterogeneity of detoxifying enzymes and effects of ethanol. Alcohol Alcohol. Suppl. 1, 235-239.

[56] Ketterer, B. (1986) Detoxication reactions of glutathione and glutathione transferases. Xenobiotica 16, $957 \sim 973$.

[57] Ketterer, B., Taylor, J.B., Meyer, D.J., Coles, B., Pemble, S., Cowell, I.G. and Dixon, K. Glutathione S-transferases: structure and function, in: Taniguchi, N., Higashi, T., Sakamoto, Y. and Meister, A. (eds.) Glutathione centennial: molleculare perspectives and clinical implications, Academic Press, San Diego, 1989, pp. $241-257$.

[58] Kilpikari, I. and Savolaimen, H. (1984) Decreased erythrocyte glutathione Stransferase activity in rubber workers. Int. Arch. Environ. Health 53, 299-302.

[59] Kitagawa, 'T., Inai, F. and Sato, K. (1980) Reevaluation of $\gamma$-glutamyltranspeptidase activity in periportal hepatocytes of rats with age. Gann. 71, 362-366.

[60] Klaassen, C.D., Amdur, M.O. and Doull J. (eds.) Casarett and Doull's Toxicology; The basic science of poisons, MacMillan Publishing Company, New York, 1986. 
[61] Kondo, T. and Kawakami, Y. Glutathione disulfide-stimulated $\mathrm{Mg}^{2 *}$-ATPase of human ery throcytes. in: Taniguchi, N., Higashi, T., Sakamoto, Y. and Meister, A. (eds.) Glutathione centennial: moleculare perspectives and clinical implications, Academic Press, San Diego, 1989, pp. 369-380.

[62] Kuijk van, F.J.G.M., Sevanian, A., Handelman, G.J. and Dratz, E.A. (1987) A new role for phospholipase $A_{2}$ : protection of membranes from lipid peroxidation damage. T.T.B.S. 12, 31-34.

[63) Kurita, H. (1967) Experimental studies on methyl-ethyl-ket-oxime toxicity. Nagoya J. Med. Sci. 29, 393-418.

[64] LaBelle, E.F., Singh, S.V., Srivastava, S.K. and Awasthi, Y.C. (1986) Dinitrophenyl glutathione efflux from human erythrocytes is primary active ATP. dependent transport. Biochem. J. 238, 443-449.

[65] Lewalter, J. and Korallus, U. (1985) Blood protein conjugates and acetylation of aromatic amines. Int. Arch. Occup. Environ. Health 56, 179-196.

[66] Lo Jacono, F., Stecca, C. and Duverger, M. (1992) Mutagenic activation of benzo[a]pyrene by human red blood cells. Mutat. Res. 268, 21-26.

[67] López-Barea, J., Bấrcena, J.A., Bocanegra, J.A., Florindo, J., García-Alfonso, C., López-Ruiz, A., Martinez-Galisteo, E. and Peinado, J. Structure, mechanism, functions, and regulatory properties of glutathione reductase, in: Viña, J. (ed.) Glutathione: metabolism and physiological functions, CRC Press, Boca Raton, 1990, pp. 105-116.

[68] Maede, Y., Kasai, N. and Taniguchi, N. (1982) Hereditary concentration of glutathione in canine erythrocytes associated with high accumulation of glutamate, glutamine, and aspartate. Blood 59, 883-889.

[69] Mannervik, B. and Danielson, U.H., (1988) Glutathione transferases; structure and catalytic activity. CRC Crit. Rev. Biochem. 23, 283-337.

[70] Mannervik, B., Widersten, M. and Board, P.G. Glutathione-linked enzymes in cletoxication reactions, in: Taniguchi, N., Higashi, T., Sakamoto, Y. and Meister, A. (eds.) Glutathione centennial: moleculare perspectives and clinical implications, Academic Press, San Diego, 1989, pp. 23-34.

[71] Maxfield, M.E., Barnes, J.R., Azar, A. and Trochimowicz, H.T. (1975) Urinary excretion of metabolite following human exposure to dimethylformamide or dimethylacetamide. J. Occup. Med. 17, 506-511.

[72] Meyer, D.`., Coles, B., Pemble, S.E., Gilmore, K.S., Fraser, G.M. and Ketterer, B. (1991) Theta, a new class of glutathione transferases purified from rat and man. Bioclem. J. 274, 409-414.

[73] Mieyal, J.J., Starke, D.W., Grawina, S.A., Dothey, C. and Chung, J.S. (1991) Thioltransferase in thuman red blood cells: purification and properties, Biochem. istry $30,6088-6097$.

[74] Mieyal, J.J., Starke, D.W., Gravina, S.A. and Hocevar, B.A. (1991) Thioltransferase in human red blood cells: kinetics and equilibrium, Biochemistry $30,8883-$ 8891.

[75] Morgenstern, R. and Depierre, J.W. Membrane-bound glutathione transferases, in: Sies, H. and Ketterer, B. (eds.) Glutathione conjugation; mechanisms and biological significance, Academic Press, London, 1988, pp. 157-174. 
[76] Nagasaka, Y., Fujii, S. and Kaneko, T. (1989) Effects of high glucose and sorbitol pathway on lipid peroxidation of erythrocytes. Horm. Metabol. Res. 21, 275-276.

[77] Neumann, H-G. (1988) Biomonitoring of aromatic amines and alkylating agents by measuring hemoglobin adducts. Int. Arch. Occup. Environ. Health 60, 151155.

[78] Norppa, H., Vainio, H. and Sorsa, M. (1983) Metabolic activation of styrene by erythrocytes detected as increased sister chromatid exchanges in cultured human lymphocytes. Cancer Res. 43, 3579-3582.

[79] Osterman-Golkar, S., Bailey, E., Farmer, P.B., Gorf, S.M and Lamb, J.H. (1984) Monitoring exposure to propylene oxide through the determination of hemoglobin alkylation. Scand. J. Work Environ. Health 10, 99-102.

[80] Peter, H., Deutschmann, S., Reichel, C. and Hallier, E. (1989) Metabolism of methyl chloride by human erythrocytes. Arch. Toxicol. 63, 351-355.

[81] Reed, D.J. (1990) Glutathione: Toxicological implications. Annu. Rev. Pharmacol. Toxicol. 30, 603-631.

[82] Richman, P.G. and Meister, A. (1975) Regulation of $\gamma$-glutamyl-cysteine synthetase by nonallosteric feedback inhibition by glutathione. J. Biol. Chem. 250, $1422-1426$.

[83] Romero, F.J. and Galaris, D. Compartimentation of cellular glutathione in mitochondrial and cytosolic pools, in: Viña, J. (ed.) Glutathione: metabolism and physiological functions, CRC Press, Boca Raton, 1990, pp. 30-38.

[84] Sabbioni, G. and Neumann, H.G. (1990) Quantification of haemoglobin binding of 4,4'-methylenebis(2-chloroaniline)(MOCA) in rats. Arch. Toxicol. 64, 451-458.

[85] Schön, M.R., Kauffman, F.C. and Thurman, R.G. (1988) Selective depletion and measurement of glutathione in periportal and pericentral regions in the perfused rat liver. Toxicol. Lett. 42, 265-272.

[86] Schröder, K.R., Hallier, E. and Bolt, H.M (1992) Dissociation of a new glutathione S-transferase activity in human erythrocytes. Biochem. Pharmacol. 43, $1671-1674$.

[87] Scott, M.D., Zuo, L., Lubin, B.H. and Chiu, D.T.-Y. (1991) NADPH, not glutathione, status modulates oxidant sensitivity in normal and glucose 6-phophate dehydrogenase-deficient erythrocytes. Blood 77, 2059-2064.

[88] Seibert, B., Oesch, F. and Steinberg, P. (1989) Distribution and induction of cytochrome P-450 and two cytochrome P-450 dependent monooxygenase activities in rat liver parenchymal cell populations separated by centrifugal elutriation. Arch. "Toxicol. 63, 18-22.

[89] Shan, X., Yee Aw, T. and Jones, D.P. (1990) Glutathione-dependent protection against oxidative injury. Pharmac. Ther. $47,61-71$.

[90] Shimiza, M. and Morita, S. (1990) Effects of fasting on cadmium toxicity, glutam thione metabolism and metallothionein synthesis in rats. Toxicol. Appl. Pharmac. $103,28-39$.

[91] Sies, H. Intracellular effects of glutathione conjugates and their transport from the cell, in: Sies, H. and Ketterer, B. (eds.) Glutathione conjugation; mechanisms and biological significance, Academic Press, London, 1988, pp. 175-192. 
[92] Sies, H., Akerboom, T. and Ishikawa, T. Glutathione conjugates: transport from the cell and irtracellular effects, in: Taniguchi, N., Higashi, T., Sakamoto, Y. and Meister A. (eds.) Glutathione centennial: moleculare perspectives and clinical implications, Academic Press, San Diego, 1989, pp. 357-367.

[93] Sinnhuber, R.O. and Yu, T.C. (1957) 2-Thiobarbituric acid method for the measurement of rancidity in fishery products; II The quantitative determination of malonaldehyde. Food Technol. 12, 9-12.

[94] Smith, M.T., Loveridge, N., Wills, E.D. and Chayen, J. (1979) The distribution of glutathione in the rat liver lobule. Biochem. J. 182, 103-108.

[95] Smith, M.T. and Wills, E.D. (1981) Effects of dietary lipid and phenobarbitone on the distribution and concentration of cytochrome P-450 in the liver studied by quantitative cytochemistry. F.E.B.S. Lett. 127, 33-36.

[96] Snijder, L.M., Leb, L., Piotrowski, J, Sauberman, N., Liu, S.C. and Fortier, N.L. (1983) Irreversible spectrin-haemoglobin crosslinking in vivo: a marker for red cell senescence. Br. J. Haematol. 53, 379-384.

[97] Speisky, H., Lindros, K.O., Varghese, G., Gunnasekare, A., Orrego, H. and Israel, Y. (1985) Acinar distribution of $\gamma$-glutamyltransferase and glutathione in rat liver. Effect of acute ethanol administration. Hepatology 5, 962.

[98] Srivastava, S.K. (1977) Absence of $\gamma$-glutamyl transpeptidase and the role of GSSG transport in the turnover of GSH in erythrocytes. Blood 49, 668-669.

[99] Srivastava, S.K., Awasthi, Y.C., Miller, S.P., Yoshida, A. and Beutler, E. (1976) Studies on $\gamma$-glutamy』 transpeptidase in human and rabbit erythrocytes. Blood $47,645-650$.

[100] Steinberg, P., Seibert, B. and Oesch, F. Separation and biochemical characterization of rat. liver parenchymal cell subpopulations, in: Roberfroid, M.B. and Preat, V. (eds.) Experimental hepatocarcinogenesis, Plenum publishing corporation, New York, 1988, pp. 257-265.

[101] Stillwell, W.G., Bryant, M.S. and Wishrok, J.S. (1987) GC/MS analysis of biologically important aromatic amines. Application to human dosimetry. Biomed. Environ. Mass Spec. 14, 221-227.

[102] Stryer, L., Biochemistry, W.H. Freeman and company, New York, 1988, pp. 349438.

[103] Sugawara, E., Nakamura, K., Miyake, T., Fukumura, A. and Seki, Y. (1991) Lipid peroxidation and concentration of glutathione in erythrocytes from workers exposed to lead. Br. J. Indus. Med. 48, 239-242.

[104] Tan, K.H., Meyer, D.J., Belin, J. and Ketterer, B. (1984) Inhibition of microsomal lipid peroxidation by glutathione and glutathione transferases $\mathrm{B}$ and $\mathrm{AA}$; role of endogenous phospholipase $A_{2}$. Biochem. J. 220, 243-252.

[105] Timbrell, J.A. (1991) Principles of biochemical toxicology, Taylor and Francis, London, pp. 77-82.

[106] Tomoda, A., Imoto, M., Hirano, M. and Yoneyama, Y. (1979) Analysis of Metform haemoglobins in human erythrocytes of normal adults and of a patient with hereditary methaemoglobinaemia due to deficiency of NADH-cytochrome $b_{5}$ reductase. Biochem. J. 181, 505-507. 
[107] Tomokuni, K., Ichiba, M., Hirai, Y., Hasegawa, T. and Sugimoto, $\mathbb{K}$. (1988) Relationship between inhibition of erythrocyte pyrimidine 5'-nucleotidase activity and biological response for porphyrin metabolism in workers occupationally exposed to lead. Int. Arch. Environ. Health 60, 431-436.

[108] Törnqvist, M., Osterman-Golkar, S., Kantiainen, A., Jensen, S., Farmer, P.B. and Ehrenberg, L. (1986) Tissue doses of ethylene oxide in cigarette smokers determined from adduct levels in hemoglobin. Crarcinogenesis 7, 1519-1521.

[109] Tursi, F., Samaia, M., Salmona, M. and Belvedere, G. (1983) Styrene oxidation to styrene oxide in human erythrocytes is catalyzed by oxyhemoglobin. Experientia $39,593-594$.

[110] Uhlig, S. and Wendel, A. (1992) The physiological consequences of glutathione variations. Life Sci, 51, 1083-1094.

[111] Vaänäen, H. (1986) The distribution of cytochrome P-450 mediated drug oxidation and glutathione in periportal and perivenous rat hepatocytes after phenobarbital treatment. J. Hepatol. 2, 174-181.

[112] Wanatabe, J., Kanai, K. and Kanamura, S. (1989) A new microphotometric method for measurement of cytochrome P-450 in sections of liver. J. Histochern. Cytochem. 37, 1257-1263.

[113] Weed, R.I. and Reed, C.F. (1966) Membrane alterations leading to red cell destruction. Am. J. Med. 41,681-698.

[114] Winter, C.G. and Christensen, H.N. (1964) Migration of amino acids across the membrane of the human erythrocyte. J. Biol. Chem. 239, 872-878.

[115] Yubisui, T., Matsuki, T., Tanishima, K., Takeshita, M. and Yoneyama, Y. (1977) NADPH-flavin reductase in human erythrocytes and the reduction of methemoglobin through flavin by the enzyme. Biochem. Biophys. Res. Comm. 76, $174-182$.

[116] Yubisui, T., Takeshita, M. and Yoneyama, Y. (1980) Reduction of methemoglobin through flavin at the physiological concentration by NADPH-flavin reductase of human erythrocytes. J. Biochem. 87, 1715-1720. 

Chapter 2

Glutathione depletion in human erythrocytes as an indicator for microsomal activation of cyclophosphamide and 3-hydroxyacetanilide

Palmen, N.G.M. and Evelo, C.T.A.

University of Limburg, Department of Pharmacology, Section Toxicology, PO Box 616, 6200 MD Maastricht, The Netherlands.

Published in: Toxicology 84 (1993) 157-170. 


\section{Summary}

A modal system for the detection of reactive metabolites, using gluta thione depletion after microsomal activation, has been described previously. We developed a battery of complementary test systems using rat liver microsomes for metabolism and aqueous glutathione solutions, human erythrocytes or hemolysate derived there from, as target. Reactive metabolite formation and the ability of metabolites to pass the erythrocyte membrane were tesited wing 3-hydroxyacetanilide (3-HAA) and cyclophosphamide (CP) as substrates. Neither unchanged 3-HAA nor CP depleted glutathione in erythrocytes or in aqueous reduced glutathione solutions (GSH solutions). Addition of normal rat liver micro somes or liver microsomes from rats pretreated with phenobarbital (PB microsomes) induced a 3-HAA/CP concentration-dependent glutathione depletion in both systems. With PB microsomes, higher depletions were found. While anchanged $3 \mathrm{HAA}$ did not deplete aqueous GSH solutions or glutathione in erythrocytes, a significant depletion in hemolysate wras found. The results indicate that both $\mathrm{CP}$ and 3-HAA metabolites are able to pass through the erythrocyte membrane. While both substances can metabolically be activated by rat liver microsomes, only 3-HAA can be activated by soluble factors in erythrocytes. However, unchanged 3 HAA has no effect on GSH in erythrocytes. This might be caused by an inability of unchanged 3-HAA to enter the erythrocyte. More generally, an adequate combination of the test systems described can be used to detect (a) the reactivity of unchanged substances and their metabolites, and (b) the ability of unchanged substances and their reactive metabolites to pass through the erythrocyte membrane.

\section{Introduction}

The tripeptide glutathione acts as an efficient scavenger of reactive electrophilic substances in conjunction with glutathone-dependent enzymes $[6,25,26,31]$. The glutathione thiol has a higher affinity for reactive (electrophilic) compounds than protein thols [8] or nucleophilic centers in DNA [5] and it also protects protein thiols against oxidation $[6,31]$. Depletion of intracellular glutathione will have consequences for these protective functions.

The liver is susceptible to glutathione depletion, as this organ has a very high phase I metabolic activity, often leading to the formation of reactive metabolites [17]. Hepatocytes have a high glutathione concentration (10-12 mM) [22]. Next to the liver, blood also contributes to glutathione-dependent metabolism. The role of blood is important because of its volume, the glutathione concentration ( $2 \mathrm{mM}$ ) in the erythrocytes and its presence all over the body. The glutathione concentration in human erythrocytes is much higher than in plasma. [26]. 
The contribution of erythrocytes to the protection against reactive metabolites depends on the ability of metabolites to pass the erythrocyte membrane [10].

The aim of the present study was to develop a system able to discriminate between:

1. substances which react with GSH without preceding metabolic activation and substances which need metabolic activation; and

2. substances or reactive metabolites able and unable to pass the erythrocyte membrane.

To study these objectives several glutathione sources were used, i.e., aqueous reduced glutathione solutions (GSH solutions), human erythrocytes and hemolysate derived there from, incubations with and without rat liver microsomes (normal or phenobarbital pretreated rat liver microsomes) were compared and 3-HAA and CP, two test compounds which are known to deplete GSH only after metabolic activation [13], were used to test our model.

The sum of both reduced and oxidized glutathione was determined (= total glutathione (GT)). The loss of GT (GT depletion) as a consequence of the incubation of 3-HAA or CP with a metabolizing system was used as a measure for reactive metabolite formation.

\section{Materials and Methods}

\section{Chemicals}

Glucose-6-phosphate dehydrogenase (G6PDH; EC 1.1.1.49), glutathione reductase (EC 1.6.4.2), Glucose-6-phosphate (G6P), NADP, cyclophosphamide (CP; CAS: 6055-19-2) and 3-hydroxyacetanilide (3-HAA; CAS: 621-42-1) were obtained from Sigma (St. Louis, MO, USA). Water was demineralized and microfiltrated before use. All other chemicals were of analytical grade quality.

\footnotetext{
Animals

Male Brown Norway rats (BN/M) of about 250 grams were used. A non-albino strain was chosen as biotransformation of xenobiotics is lower in albino strains [29]. Housing was as previously described [27]. When liver microsomes from rats pretreated with phenobarbital (PB microsomes) were used, phenobarbital was added to the drinking water $\left(1 \mathrm{~g}^{-l^{-1}}\right)$ during one week prior to the preparation of microsomes.
} 


\section{Preparation of microsomal activating system}

Animals were anesthetized using pentobarbital (Narcovet ${ }^{T M} ; 0.2 \mathrm{ml} / 100 \mathrm{~g}$ rat). Livers were removed and homogenized in three volumes of ice-cold phosphate buffer ( $50 \mathrm{mM}$ and $\mathrm{pH} 7.4$ ), using glass Potter tubes with a Teflon pestle. The homogenate was centrifuged at $9000 \times \mathrm{g}$ for 20 minutes at $4^{\circ} \mathrm{C}$. The microsomal fraction was sedimented from the supernatant by 40 minutes centrifugation at $110000 \times \mathrm{g}$ at $4^{\circ} \mathrm{C}$. The pellet was resuspended in the same ice-cold buffer and diluted so that the microsomal fraction of $3 \mathrm{~g}$ liver was suspended in $1 \mathrm{ml}$ buffer. Microsomal activating system was composed of microsomes (final protein concentration $1 \mathrm{mg} \cdot \mathrm{ml}^{-1}$ ) and NADPH regenerating system (0.5 mM NADP, $5.0 \mathrm{mM}$ $\mathrm{MgCl}_{2}, 5.0 \mathrm{mM} \mathrm{G6P}$ and $\left.1.0 \mathrm{U} \cdot \mathrm{ml}^{-1} \mathrm{G} 6 \mathrm{PDH}\right)$

\section{Preparation of erythrocytes and lysate}

Human blood was obtained from the Red Cross Blood Bank "Zuid Limburg", collected in sterile vacuum EDTA vessels and kept overnight at $4^{\circ} \mathrm{C}$. Erythrocytes were pooled, washed three times using saline and diluted three times in $100 \mathrm{mM} \mathrm{Na} 2 \mathrm{HPO}_{4} / \mathrm{KH}_{2} \mathrm{PO}_{4}$ buffer (final hemoglobin concentration about $\left.100 \mathrm{~g}^{-1} \mathrm{l}^{-1}\right)$. Lysate was prepared from packed erythrocytes by dilution with icecold water (1:2). The cellular debris was removed by centrifugation ( 5 minutes, $2000 \times \mathrm{g})$.

\section{Incubations}

Human erythrocytes (final $\mathrm{Hb}$ concentration $23 \mathrm{~g} \cdot \mathrm{l}^{-1}$ ) or aqueous GSH solutions (final concentration $450 \mu \mathrm{M}$ ) were suspended in $100 \mathrm{mM} \mathrm{Na}_{2} \mathrm{HPO}_{4} / \mathrm{KH}_{2} \mathrm{PO}_{4}$ buffer (final volume $3 \mathrm{ml}$ ). Incubations were performed both with and without microsomal activating system. Microsomes of both normal and phenobarbital pretreated animals were tested. The complete test system without test substance was preincubated for 5 minutes at $37^{\circ} \mathrm{C}$ in a shaking waterbath $(80 \mathrm{rev} . / \mathrm{min})$. Thereafter, $1 \mathrm{ml} 3-\mathrm{HAA}$ or $\mathrm{CP}$ (final concentration between 0 and $2 \mathrm{mM}$ ) was added and incubated for 1 hour. All experiments were performed three times on different days and with different blood samples. Each day, all incubations were performed in triplicate. Separate experiments were performed for test compound concentrations between 0 and $0.5 \mathrm{mM}$ and between 0.5 and $2.0 \mathrm{mM}$.

To verify metabolic activity of the erythrocytes themselves, GT depletion in hemolysate was also tested. After preincubating hemolysate for 5 minutes at $37^{\circ} \mathrm{C}$ in a shaking waterbath $(80 \mathrm{rev} / \mathrm{min}) 1 \mathrm{ml} \mathrm{3-HAA}$ or CP (final concentrations $0,0.51 .0$ and $2.0 \mathrm{mM}$ ) was added and incubated for 1 hour. After 1 hour incubation, the reaction was stopped by the addition of an equal volume of $5 \%$ $(w / v)$ trichloroacetic acid (TCA). Erythrocytes were packed before the addition of TCA by centrifugation ( 5 minutes, $2000 \times \mathrm{g}$ ). The degree of concentration was 
determined by measuring hemoglobin concentrations of the test incubate before and after concentration. The amount of erythrocytes pipetted for determination of the hemoglobin concentration was weighed.

\section{Analytical procedures}

GT in the TCA supernatants was determined after dilution (9-fold) with buffer by the cyclic oxidation reduction method described by Anderson [2]. Microsomal protein content was determined according to Lowry et al. [24]. Hemoglobin concentrations were determined by measuring cyanmethemoglobin according to van Kampen and Zijlstra [35].

\section{Statistical analysis}

GT depletion was analyzed by linear regression using a model including day of experiment, concentration, metabolizing system and concentration times metabolizing system as explanatory variables. Using that model, we tested whether slopes of $\mathrm{GT}$ depletion versus concentration were significant for each of the metabolizing systems and whether these slopes were different from each other, correcting for the day of the experiment.

\section{Results}

\section{3-Hydroxyacetanilide}

Depletion of GT by addition of 3-HAA with and without microsomal activation is shown in Figures 2.1 and 2.2 for aqueous GSH solutions and erythrocytes, respectively. Without metabolic activating system, GT was not depleted in both systems. Microsomal activation of 3-HAA resulted in GT depletion, both in incubations with aqueous GSH solutions and in erythrocytes. This depletion was stronger when $\mathrm{PB}$ microsomes were used.

For aqueous GSH solutions, the depletion increased from $14 \quad(S D=23)$ nmoles $\cdot \mathrm{ml}^{-1} \mathrm{~h}^{-1}$ at $0.1 \mathrm{mM} 3-\mathrm{HAA}$ to $70(\mathrm{SD}=38) \mathrm{nmoles} \cdot \mathrm{ml}^{-1} \mathrm{~h}^{-1}$ at $2.0 \mathrm{mM}$ $(p=0.0001)$ with normal microsomes and from $67(\mathrm{SD}=30)$ nmoles $\cdot \mathrm{ml}^{-1} \mathrm{~h}^{-1}$ at $0.1 \mathrm{mM} 3-\mathrm{HAA}$ to $91(\mathrm{SD}=16) \mathrm{nmoles} \cdot \mathrm{ml}^{-1} \mathrm{~h}^{-1}$ at $2.0 \mathrm{mM}$ with $\mathrm{PB}$ microsomes $(p=0.0001)$. GT depletion in incubations with aqueous GSH solutions and $\mathrm{PB}$ microsomes was maximal at a 3 -HAA concentration of about $0.3 \mathrm{mM}$ (99 nmoles $\cdot \mathrm{ml}^{-1} \mathrm{~h}^{-1}, \mathrm{SD}=27$ ). For erythrocytes, GT depletion was also statistically significant and was $24(\mathrm{SD}=1)$ and $61(\mathrm{SD}=3)$ nmoles $\mathrm{ml}^{-1} \mathrm{~h}^{-1}$ at $2 \mathrm{mM}$ 3-HAA with normal $(p=0.0040)$ and PB microsomes $(p=0.0001)$, respectively.

GT depletions using normal microsomes were significantly higher compared to incubations without microsomes for both aqueous GSH solutions $(p=0.0004)$ and 


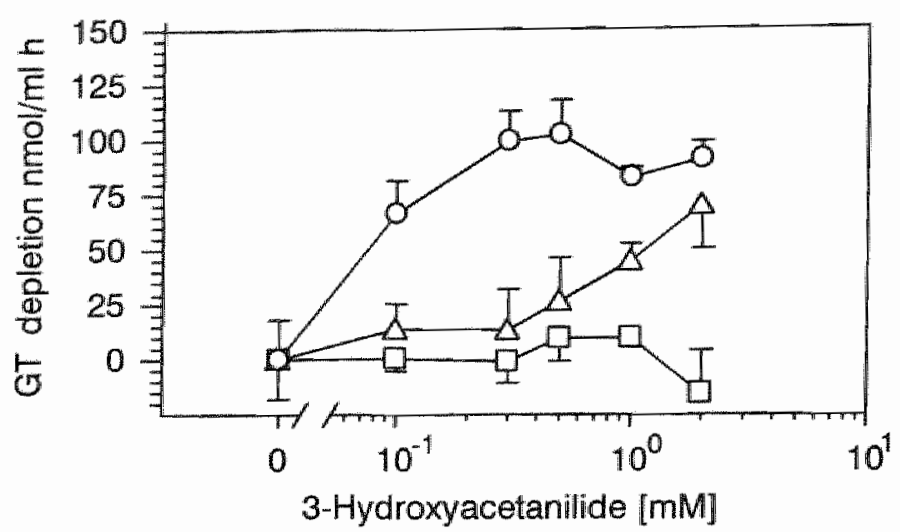

Figure 2.1: GT depletions in incubations of aqueous GSH solutions with 3-HAA. Microsomes of PB pretreated (O) and untreated $(\Delta)$ rats were used. Controls $(\square)$ were incubated without microsomes. The means of $\mathrm{GT}$ depletions and half the SD of GT concentrations are shown. The number of experiments was $\pi=6$ for 0 and $0.5 \mathrm{mM} \mathrm{3-HAA}$ and $\mathrm{n}=3$ for the other concentrations tested. GT depletion was significantly related to the 3-HAA concentration, for both normal $(p=0.0001)$ and PB microsomes $(p=0.0001)$. Compared to incubations without microsomes, GT depletions were significantly higher when normal $(p=0.0004)$ or $\mathrm{PB}(p=$ 0.0002 ) microsomes were used.

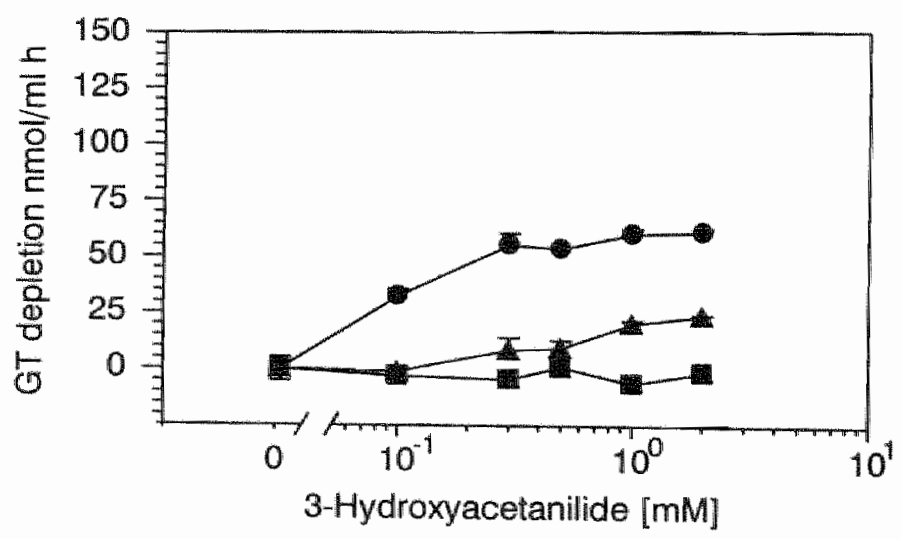

Figure 2.2: GT depletions in incubations of human erythrocytes with 3-HAA. Microsomes of $\mathrm{PB}$ pretreated ( and untreated (A) rats were used. Controls ( were incubated without microsomes. The means of GT depletions and half the SD of $\mathrm{GT}$ concentrations are shown. The number of experiments was $n=5$ for 0 and $0.5 \mathrm{mM} 3-\mathrm{HAA}$ and $\mathrm{n}=3$ for the other concentrations tested. GT depletion was significantly related to the 3-HAA concentration, for both normal $(p=0.0040)$ and $\mathrm{PB}$ microsomes $(p=0.0001)$. Compared to incubations without microsomes, GT depletions were significantly higher when normal $(p=0.0329)$ or PB $(p=$
$0.0002)$ microsomes were used. 
erythrocytes $(p=0.0329)$. At 3-HAA concentrations between 0 and $0.5 \mathrm{mM}$, GT depletions in incubations with PB microsomes were higher than GT depletions in incubations with normal microsomes for both aqueous GSH solutions $(p=0.0001)$ and erythrocytes $(p=0.0001)$; above $0.5 \mathrm{mM}$ saturation of 3-HAA metabolism is reached in $\mathrm{PB}$ microsomes.

A concentration-dependent GT depletion could be observed when lysate was incubated with 3-HAA $(p=0.0018)$. Absolute depletions were $12(\mathrm{SD}=22)$, $25(\mathrm{SD}=19)$ and $36(\mathrm{SD}=6)$ nmoles $\cdot \mathrm{m}^{-1} \mathrm{~h}^{-1}$ with $0.5,1.0$ and $2.0 \mathrm{mM}$ 3-HAA, respectively.

\section{Cyclophosphamide}

Figures 2.3 and 2.4 show depletion of GT by addition of $\mathrm{CP}$ with and without microsomal activation for aqueous GSH solutions and erythrocytes, respectively. In the absence of microsomal activation, CP induced no GT depletion in incubations in which aqueous GSH solutions or erythrocytes were used. Microsomal activation of CP resulted in GT depletion both in incubations with aqueous GSH solutions and in erythrocytes. GT depletion was higher when PB microsomes were used. GT was not depleted when lysate was incubated with CP.

GT depletion in incubations with aqueous GSH solutions increased from 16 $(\mathrm{SD}=23) \mathrm{nmoles} \cdot \mathrm{ml}^{-1} \mathrm{~h}^{-1}$ at $0.5 \mathrm{mM} \mathrm{CP}$ to $105(\mathrm{SD}=8)$ mmoles $\mathrm{ml}^{-1} \mathrm{~h}^{-1}$ at $2 \mathrm{mM}(p=0.0001)$ with normal microsomes and from $93(\mathrm{SD}=44)$ nmoles $\cdot \mathrm{ml}^{-1} \mathrm{~h}^{-1}$ at $0.5 \mathrm{mM} \mathrm{CP}$ to $218(\mathrm{SD}=30) \mathrm{nmoles} \cdot \mathrm{ml}^{-1} \mathrm{~h}^{-1}$ at $2.0 \mathrm{mM}$ with PB microsomes $(p=0.0001)$. For erythrocytes, GT depletions increased from 35 $(\mathrm{SD}=11)$ nmoles $\mathrm{ml}^{-1} \mathrm{~h}^{-1}$ at $0.5 \mathrm{mM} \mathrm{CP}$ to $92(\mathrm{SD}=6) \mathrm{at} 2 \mathrm{mM}(p=0.000 \mathrm{~L})$ with normal microsomes and from $96(\mathrm{SD}=12)$ nimoles $\mathrm{ml}^{-1} \mathrm{~h}^{-1}$ at $0.5 \mathrm{mMCP}$ to $90(\mathrm{SD}=13)$ nmoles $\cdot \mathrm{ml}^{-1} \mathrm{~h}^{-1}$ at $2 \mathrm{mM}(p=0.0001)$ with $\mathrm{PB}$ microsomes. In incubations with erythrocytes, all glutathione was consumed at $0.5 \mathrm{mM} \mathrm{CP}$ when PB microsomes were tested. In inculbations with normal microsomes, GT depletion in incubations with erythrocytes was comparable with incubations with aqueous GSH solutions.

GT depletions in incubations with normal microsomes were significantly higher compared to incubations without microsomes for erythrocytes $(p=0.0001)$ and aqueous GSH solutions $(p=0.0017)$, respectively. At CP concentrations between 0 and $0.5 \mathrm{mM}$, GT depletions in incubations with $\mathrm{PB}$ microsomes were higher than GT depletions in incubations with normal microsomes for both aqueous GSH solutions $(p=0.0001)$ and erythrocytes $(p=0.0001)$, above $0.5 \mathrm{mM}$ saturation of CP metabolism is reached in PB microsomes. 


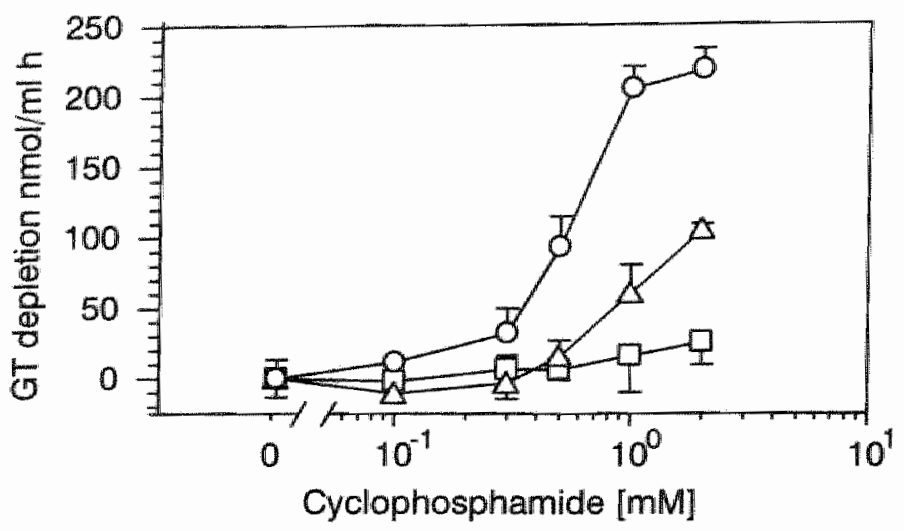

Figure 2.3: GT depletions in incubations of aqueous GSH solutions with CP. Microsomes of $\mathrm{PB}$ pretreated $(O)$ and untreated $(\Delta)$ rats were used. Controls $(\square)$ were incubated without microsomes. The means of GT depletions and half the SD of $\mathrm{GT}$ concentrations are shown. The number of experiments was $\mathrm{n}=5$ for 0 and $0.5 \mathrm{mM} 3-\mathrm{HAA}$ and $\mathrm{n}=3$ for the other concentrations tested. GT depletion was significantly related to the 3-HAA concentration, for both normal $(p=0.0001)$ and PB microsomes $(p=0.0001)$. Compared to incubations without microsomes, GT depletions were significantly higher when normal $(p=0.0017)$ or $\mathrm{PB}(p=$ 0.0001) microsomes were used.

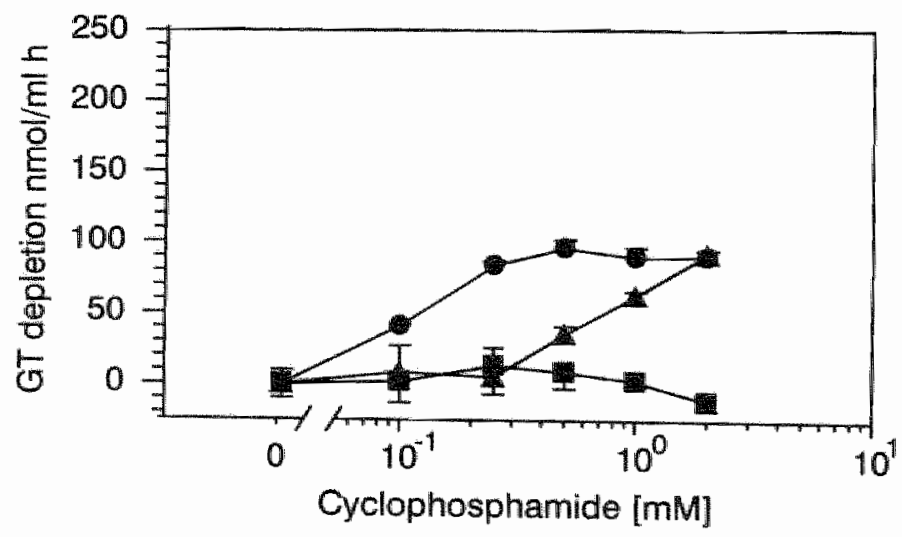

Figure 2.4: GT depletions in incubations of human eryihrocytes with CP. Microsomes of PB pretreated (-) and untreated (4) rats were used. Controls (1) were incubated without microsomes. The means of $\mathrm{GT}$ depletions and half the $\mathrm{SD}$ of $\mathrm{GT}$ concentrations are shown. The number of experiments was $\mathrm{n}=6$ for 0 and $0.5 \mathrm{mM} 3$.HAA and $\mathrm{n}=3$ for the other concentrations tested. GT depletion was significantly related to the 3 -HAA concentration, for both normal $(p=0.0001)$ and PB microsiomes $(p=0.0001)$. Compared to incubations without microsomes, $G T$ depletions were significantly higher when normal $(p=0.0001)$ or PB $(p=0.0001)$ microsomes were used. 


\section{Discussion}

GSH plays a key role in the detoxification of electrophiles. It also reduces protein and hemoglobin thiols and prevents the formation of hemoglobin and DNA adducts [8]. GSH depletion has been used previously to quantify the formation of reactive metabolites in an in vitro test system containing aqueous GSH solutions and microsomes $[13,15,14]$. We used a similar system for detection of reactive metabolites formed from 3-HAA and CP. In addition, the ability of the metabolites formed to enter a target cell was evaluated. For this purpose GT depletion in human erythrocytes added to the microsomal system was determined. Finally, the metabolic activity of the erythrocytes themselves, which contain both oxygenase $[7,32]$ and glutathione $S$-transferase activity $[9]$, was tested using hemolysate.

In this study 3-hydroxyacetanilide (3-HAA), an analogue of paracetamol with antipyretic and analgetic properties [33] and cyclophosphamide (CP), which is used as a chemotherapeutic agent and as an immunosuppressor [28], were tested. The liver is the principal site for biotransformation of CP [11,34] and 3-HAA [33]. CP is metabolised by hepatic microsomal enzymes to 4-hydroxycyclophosphamide. This initial transformation is rate limiting[19]. From 4-hydroxycyclophosphamide, equimolar amounts of phosphoramide mustard and acrolein are produced [20]. Acrolein forms a conjugate with GSH and is excreted into the urine as 3-hydroxypropylmercapturic acid. The rate of biotransformation of CP increased $2-3$-fold in patients treated with phenobarbital $[4,21]$ and it was concluded that the metabolism of $\mathrm{CP}$ is mediated by liver mixed-function oxidases. Pretreatment of rats with SKF 252A [34] and cimetidine [3] inhibited CP metabolism, also indicating that CP metabolism is mediated by cytochrome P-450. 3-HAA is metabolised by mouse hepatic microsomes to $2^{\prime}, 5^{\prime}$-dihydroxyacetanilide (DHAA; note that the 3'-OH group is renumbered to 5 ' in this structure), 3',4'-DHAA and 2',3'-DHAA. The 2',5'-DHAA metabolite is oxidized further to an electrophilic semiquinone and/or quinone species [33].

GSH plays a significant role in protection against CP and 3-HAA toxicity and covalent binding. $C P$ toxicity in rats pretreated with $L$-buthionine- $S R$-sulfoximine which inhibits GSH synthesis, was enhanced [12]. CP caused a dose-dependent reduction in pulmonary GT stores in rats [1]. Covalent binding to macromolecules after CP treatment in mice was enhanced by diethylmaleate (a GSH depletor) and blocked by cysteine [18], indicating that GSH plays a protective role against covalent binding of CP metabolites. In vitro, a substantial depletion of cellular GSH was accompanied by a significant reduction of tumor cell reproductive capacity [23]. In vivo administration of 3-HAA to hamsters caused a significant decrease in hepatic GT only at doses of $550 \mathrm{mg} \cdot \mathrm{kg}^{-1}$ and higher [30]. Metabolites of 3-HAA induce covalent binding to hepatic protein [30]. Cysteine and GSH were found to inhibit this binding [33].

After oxidation, erythrocyte glutathione is either excreted by erythrocytes 
or bound to proteins (mixed disulfides) [16]. Excretion of oxidized glutathione or formation of mixed disulfides in erythrocytes would be identified as depletion of total GT. Concentrations of GT in the supernatants were determined. However, no GT could be detected in these preparations, suggesting the formation of protein-glutathione mixed disulfides or glutathione-metabolite complexes.

GT was not depleted when 3-HAA or CP were incubated whith aqueous GSH solutions or erythrocytes. GT was depleted in both systems when 3-HAA or CP were tested after the addition of microsomal activating system. This indicates not only that metabolic activation is necessary before glutathione depletion can occur, but also that such an activation does not occur within the erythrocyte or that the unchanged substances are not able to enter the erythrocyte. GT concentrations in hemolysates decreased when 3-HAA was used as a test substrate. This indicates that erythrocytes contain soluble factors able to activate 3-HAA. Therefore, it is likely that 3-HAA itself is not able to enter the erythrocyte.

The addition of normal microsomes with a NADPH regenerating system to incubations with aqueons GSH solutions or erythrocytes caused GT depletions which were concentration dependent for both test compounds. Combined with the results found without added microsomes, this proves that metabolites of both 3-HAA and CP are able to pass the erythrocyte membrane and to deplete $\mathrm{GT}$. GT depletions were higher for both 3-HAA and CP when PB microsomes were tested. As CP and 3-HAA are mainly metabolised by hepatic microsomal en$2 y$ mes $[19,33]$, these results are in agreement with the literature.

GSH depletions in incubations with aqueous GSH solutions and normal or PB microsomes and $1 \mathrm{mM} 3-\mathrm{HAA}$ of 39 nmoles $\mathrm{ml}^{-1} 30 \mathrm{~min}^{-1}$ and 209 amoles $\cdot \mathrm{ml}^{-1} 30 \mathrm{~min}^{-1}$, respectively have been reported [13]. As only $200 \mu \mathrm{M}$ aqueous GSH solution was added to their incubations, GSH was totally depleted using $\mathrm{PB}$ animals. We found $\mathrm{GT}$ depletions of $45(\mathrm{SD}=16)$ nmoles $\mathrm{ml}^{-1} \mathrm{~h}^{-1}$ and $83(\mathrm{SD}=9)$ nmoles $m \mathrm{l}^{-1} \mathrm{~h}^{-1}$ for normal and $\mathrm{PB}$ microsomes, respectively, under the same conditions. The lower GT depletion velocity found for PB microsomes in the present study might be caused by the different rat strain used or differences in phenobarbital treatment regimes. GT depletions in incubations with CP ( $1 \mathrm{mM}$ ) are higher in comparison with those of Fry for both normal and $\mathrm{PB}$ microsomes. Fry reported GSH depletions of 15 and $85 \mathrm{mmoles} \cdot \mathrm{ml}^{-1} 30 \mathrm{~min}^{-1}$ for normal and $\mathrm{PB}$ microsomes, respectively. At $\mathrm{CP}$ concentrations of $1.0 \mathrm{mM}$, we found $\mathrm{GT}$ depletions of $60(\mathrm{SD}=40)$ and $205(\mathrm{SD}=31)$ mmoles $\cdot \mathrm{ml} l^{-1} \mathrm{~h}^{-1}$ respectively. This also may be a result of differences in rat strain or pretreatment conditions. The differences in relative glutathione depletions in the two studies for CP (higher in our study) and 3-HAA (lower in our study) raight indicate that CP and 3-HAA are metabolised by different P-450 isoenzymes.

In metabolically activated incubations in which $\mathrm{CP}$ was used as a test substrate, GT depletions in incubations with aqueous GSH solutions were comparable with 
incubations with erythrocytes. In contrast, GT depletions in incubations with 3-HAA were about two times lower in incubations with erytlurocytes. These results indicate that the relative intracellular concentration of reactive metabolite concentration (compared to extracellular concentrations) is higher for CP than for 3-HAA. This may be caused by a difference in uptake or in intracellular metabolism.

Based on our results, it may be concluded that while unchanged 3-HAA and CP do not deplete GT, microsomal activation products are able to enter the erythrocyte and to deplete GT. An appropriate combination of test systems as described here can be used to discriminate direct reactive substances from those which have to be metabolised, while also metabolites able to pass the (erythrocyte) cell membrane can be discriminated from others.

Acknowledgements The authors wish to thank H. Smit, J. Linssen and A. Slangen for their technical support, Dr. A. Kester for his advice in statistical analyses, Prof. P.Th. Henderson for critical reading of the paper and the Red Cross Blood Bank "Zuid Limburg" for collecting the blood samples. 


\section{Bibliography}

[1] Allen, J., Cooper, D. and Merrill, W.W. (1989) Modulation of endoperoxide product levels and cyclophosphamide-induced injury by glutathione repletion. J. Appl. Physiol. 67, $2316-2322$.

[2] Anderson, M.E. Determination of glutathione and glutathione disulfide in biolagical gamples, in: Meister, A. (ed.), Methods Enzymol, vol. 113, Academic Press, Orlando, USA, 1985, p. 548-555.

[3] Anthony, L.B., Long, Q.C. Struck, R.F. and Hande, K.R. (1990) The effect of cimetidine on cyclophosphamide metabolism in rabbits. Cancer Chemother. Pharmacol. $27,125-130$.

[4] Bagley, C.M., Bostick, F.W. and DeVita, V.T. (1973) Clinical pharmacology of cyclophosphamide. Cancer Res. 33, 226-233.

[5] Coles, B. and Ketterer, B. (1990) The role of glutathione and glutathione transferases in chemical carcinogenesis. Crit. Rev. Biochem. Mol. Biol. 25, 47-66.

[6] Deneke, S.M. and Fanburg, B.L. (1989) Regulation of cellular glutathione. Am. J. Physial. 257, L163-L173.

17] Duverger-van Bogaert, M., Crutzen-Fayt, M.C. and Stecca, C. (1991) Mutagenicity of some heterocyclic amines in salmonella typhimurium with metabolic activatilor by human red blood cell cytosol. Mutat. Res. 261, 261-265.

[8] Evelo, C.T.A and Henderson, P.Th. (1988) Influence of glutathione on the formation of cysteine alkylation products in human hemoglobin. Toxicology 52, 177-186.

[9] Evelo, C.T.A., Palmen, N.G.M., Arthur, Y. and Janssen, G.M.E. (1992) Changes in blood glutathione concentrations, and in erythrocyte glutathione reductase and glutathione S-transferase activity after running training and after participation in contests. Eur. I. Physiol. 64, 354-358.

[10] Fazi, A., Manchini, U, Piatti, E., Accorsi, A. and Magnani, M. (1991) Human red blood cells as bioreactors for the inactivation of harmful xenobiotics. Biotechnol. Appl. Biochem. 14,60-68.

[11] Foley, G.E., Friedman, O.M. and Drolet, B.P. (1961) Studies on the mechanism of action of cytoxan evidence of activation in vivo and in vitro. Cancer Res. 21 . $57-6$.

[12] Friedman, H.S., Colvin, O.M., Aisaka, K., Popp, J., Bossen, E.H., Reimer, K.A., Powell, J.B., Hilton, I., Gross, S.S., Levi, R., Bigner, D.D and Griffith, O.W. (1990) Glutathione protects cardiac and skeletal muscle from cyclophosphamideind nced toxicity. Cancer Res. 50, 2455-2462.

[13] Fry, J.R. Reactive metabolite formation in fortified liver microsomes, The ERGATT/FRAME data bank of in vitro techniques in toxicology, protocol number 10, Nottingham, England, 1989, p. 1-9.

[14] Garle, M.J. and Fry, J.R. (1989) Detection of reactive metabolites in vitro. Toxicology $54,101-110$.

[15] Garle, M.J., Khan, J. and Fry, J.R. (1988) Depletion of glutathione by the hepa totoxins paracetamol and bromobenzene, and their non-hepatotoxic analogues, in a fortified liver microsomal system. Toxicol. in Vitro 2, 247-252. 
[16] Grossman, S.J., Simson, J. and Jollow, D.J. (1992) Dapsone-induced hemolytic anemia: effect of N-hydroxy dapsone on the sulfhydryl status and membrane proteins of rat erythrocytes. Toxicol. Appl. Pbarmacol. $117,208-217$.

[17] Guengerich, F.P. and Liebler, D.C. (1985) Enzymatic activation of chemicals to toxic metabolites. Crit. Rev Toxicol. 14, 259-307.

[18] Gurtoo, H.L., Hipkens, J.H. and Sharma, S.D. (1981) Role of glutathione in the metabolism-dependent toxicity and chemotherapy of cyclophosphamide. Cancer Res. 41, 3584-3591.

[19] Hong, P.S., Srigritsanapol, A. and Chan, K.K. (1991) Pharmacokinetics of 4hydroxycyclophosphamide and metabolites in the rat. Drug Metab. Dispos. 19, $1-7$.

[20] Honjo, I., Suou, T. and Hirayama, C. (1988) Hepatotoxicity of cyclophosphamide in man: pharmacokimetic analysis. Res. Commun. Chem. Pathol Pharmacol. 61, $149-165$.

[21] Jao, J.Y., Jusko, W.J. and Cohen, J.L. (1972) Phenobarbital effects on cyclophosphamide pharmacokinetics in man. Cancer Res. 32, $2761-2764$.

[22] Ketterer, B. (1982) The role of nonenzymatic reactions of glutathione in xenobiotic metabolism. Drug Metab. Rev. 13, 161-167.

[23] Lee, F.Y.F., Flannery, D.J, and Siemann, D.W. (1991) Prediction of tumour sensitivity to 4-hydroxycyclophosphamide by a glutathione-targeted assay. Br. J. Cancer $63,217-222$.

[24] Lowry, O.H., Rosebrough, N.J., Farr, A.L. and Randall, R.J. (1951) Protein measurement with the Folin phenol reagent. J. Biol. Chem. 193, 265-275.

[25] Mannervik, B. and Danielson, U.H. (1988) Glutathione transferases: structure and catalytic activity. Crit. Rev. Biochem. 23, 283-337.

[26] Meister, A. and Anderson, M.E. (1983) Glutathione. Annu. Rev. Biochem. 52, $711-760$.

[27] Palmen, N.G.M., Evela, C.T.A., Borm, P.J.A. and Henderson, P.Th. (1992) Infuence of oxygen supply on liver condition and elimination of dimethylacetamide in the isolated perfused rat liver. Toxicol. in Vitro 6, 357-365.

[28] Patel, J.M. (1990) Metabolism and pulmonary toxicity of cyclophosphamide. Pharmacol. Ther. 47, 137-146.

[29] Prieur, D.J. (1982) Albino animals: their use and misuse in biomedical research. Comp. Pathol. Bull. 14, 1-4.

[30] Roberts, S.A., Price, V.F. and Jollow, D.J. (1990) Acetaminophen structuretoxicity studies: in wro covalent binding of a nonhepatotoxic analog, 3hydroxyacetanilide. Toxicol. Appl. Pharmacol. 105, 195-208.

[31] Sies, H. (1986) Biochemistry of oxidative stress. Angew. Chem. Int. Ed. Engl. 25, 1058-1071.

[32] Stecca, C. and Duverger-van Bogact, M. (1989) N-Demethylation reactions in intact erythrocytes and erythrocyte supernatant. Arch. Toxicol. Suppl. 13, 291293.

[33] Streeter, A.J., Bjorge, S.M., Axworthy, D.B., Nelson, S.D. and Baillie, T.A. (1984) The microsomal metabolism and site of covalent binding to protein of $3^{3}$ - 
hydroxyacetanilide, a nonhepatotoxic positional isomer of acetaminophen. Drug Metab. Dispos. 12, 565-576.

[34] Torkelson, A.R., LaBudde, J.A. and Weikel, J.H. (1974) The metabolic fate of cyclophosphamide. Drug Metab. Rev. 3, 131-165.

[35] van Kampen, E.J. and Zijlstra, W.G. (1965) Determination of hemoglobin and its derivates. Adv. Clin. Chem. 8, 141-187. 
Chapter 3

Glutathione depletion in human erythrocytes and rat liver; a study on the interplay between bio-activation and inactivation functions of liver and blood

Palmen, N.G.M. and Evelo, C.T.A.

Department of Pharmacology, Section Toxicology, University of Limburg, PO Box 616, 6200 MD Maastricht, The Netherlands. 


\section{Summary}

The interplay between bioactivation and inactivation functions of human erythrocytes and rat liver was studied. Glutathione depletion was used as a measure for the amount of GSH-reactive compound. The direct electrophiles iodoacetamide (IACA), Nethylmalleimide (NEM) and diethyl maleate (DEM), were able to deplete reduced glutathione (GSH) in both incubations with aqueous GSH solution and erythrocytes. This indicates that these compounds can pass the erythrocyte membrane. Cyclophosphamide (CP), 3-hydroxyacetanilide (3-HAA) and 2-methylfurane (2-MF) needed metabolic activation by rat liver microsomes to deplete glutathione in incubations with aqueous GSH solution or erythrocytes. In these incubations the sum of both reduced and oxidized glutathione (=total glutathione (CT)) was measured. It indicates that GSH-reactive metabolites are generated out of CP, 3-HAA and 2-MF by microsomes and that these metabolites can pass the erythrocyte nembrane. As GT depletions were higher when microsomes of phenobarbital pretreated rats were tested, the metabolites were (also) generated by phenobarbital inducable enzymes. GT was also depleted in incubations with hemolysate and 3-HAA or 2-MF, but not in incubations with aqueous GSH solution. This indicates that erythrocyte cytosol can metabolize 3HAA and $2-M F$ into GSH-reactive compounds. The pesticides monuron and monulimuron did not affect GrT concentrations when wqueous GSH solution, hemolysate or erythrocytes with or without microsomal activating system were tested. When hepatocytes were incubated with $\mathrm{CP}$ or 3-HAA ( $2 \mathrm{mM}$ ), the hepatic GT concentration was depleted (about $1.5 \mathrm{mM}$ in both cases). The hepatocytes were able to excrete GSHreactive metabolltes generated from $\mathrm{CP}$ or $3 \mathrm{HAA}(21 \%$ and $77 \%$ resp.). GT was not depleted in co-incubations of hepatocytes and erythrocytes with $3-H A A$, which may be explained by uptake limitation of the GSH-reactive metabolites into the erythrocytes.

\section{Introduction}

Reduced glutathione (GSH) and glutathone S-transferase (GST) play a key role in the in vivo detoxification of electrophiles $[6,21,22,27]$. As human erythrocytes contain a great amount of GSH (about $2 \mathrm{mM}$ ) [10] and a relatively high GST activity ( $1-6 \mathrm{U}^{-\mathrm{g}^{-1}}$ hemoglobin) [33], they may play an important role in the detoxification of electrophiles. Various electrophiles are known to cause glutathione depletion in erythrocytes in utro (GSH-reactive compounds) $[5,9,10,11,18]$. The importance of GSH in erythrocyte based detoxification mechanism, also appears from the increased ability of erythrocytes overloaded with GSH to conjugate increased amounts of the electrophile 1-chloro-2,4 dinitrobenzene for instance [10]. 
On the other hand, erythrocytes also contain mixed function oxidase (MFO) activity capable of generating GSH-reactive compounds [3]. Erythrocytes are for instance known to metabolize styrene into styrene oxide $[3,25,35]$, benzo(a) pyrene into quinones [19], heterocyclic amines into intermediates with mutagenic activity [7], to hydroxylate aniline [23], and to perform oxidative demethylation on methylaniline with concomittant release of formaldehyde [32]. Oxyhemoglobin is thought to be responsible for this mixed function oxygenase activity [3].

For most compounds the activity of the electrophilic metabolite formation is much higher in the liver [14]. Electrophilic metabolites generated by the liver may be detoxified in turn by the hepatocyte. However, electrophilic metabolites can also be excreted, enter the erythrocytes in the sinusoids, and deplete erythrocyte glutathione (GSH-reactive metabolites).

In the present investigation, attention was paid to the interplay between the bio-activation and inactivation functions of liver and blood. The main questions, approached in vitro, were as follows:

- What is the importance of bio-activating and bio-inactivating potency of red blood cells in comparison with the hepatic capacity?

- Are erythrocytes accessible for GSH-reactive electrophilic compounds?

- Is the outer cell membrane of hepatocytes a barrier for reactive metabolites formed intracellularly, or occurs intra-hepatically an effective detoxification?

We developed an incubation system using different combinations of erythrocytes, hemolysate and aqueous GSH solution with rat liver microsomes or intact hepatocytes to imitate the physiological in vivo situation as good as possible. An outline of the various experimental conditions studied in the present investigation is depicted in Figure 3.1.

Direct reactivity of the substrate $(\mathrm{X})$ against GSH was tested by means of incubations with aqueous GSH solutions (arrow 1, Figure 3.1). Metabolic activity of erythrocytes for the test substrates (mixed function oxidase (MFO) plus glutathione S-transferase (GST) was tested through comparison of incubations with aqueous GSH solution and incubations with hemolysate (arrow 2). The influence of erythrocyte integrity on uptake and metabolism of the test compounds was tested by comparison of incubations with hemolysate and erythrocytes (arrow 3). This influence may comprise of influences of the membrane on uptake and, for example, of differences in reductive capacity due to higher oxygen exposure of the hemolysates.

Biotransformation of the test compound by means of microsomal activating system (MAS) into glutathione-reactive metabolites was tested through incubations with aqueous GSH solution with (arrow 1') and without (arrow 1) MAS. These incubations were compared with incubations in which erythrocytes and MAS were combined (arrow $3^{3}$ ). 


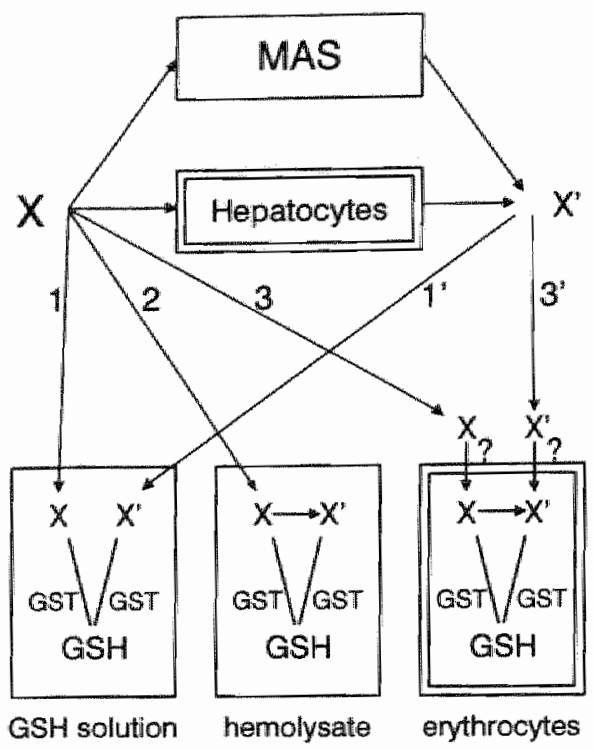

Figure 3.1: An outline of the incubations performed in this study. The picture is explained in the text.

Biotransformation of the test compound by hepatocytes (both phase I and phase II activity) was tested through depletion of hepatocyte GT. The ability of GSHreactive metabolites to pass the hepatocyte membrane was also tested. Therefore, after incubation of substrate with hepatocytes, the intact cells were removed by centrifugation and the supernatant was incubated again with GSH (arrow 1'). Co-incubations of hepatocytes and erythrocytes (arrow 3') were used to test whether metabolites generated and excreted by hepatocytes are able to enter the erythrocyte and deplete erythrocyte $\mathrm{GT}$.

The compounds we tested can be divided in direct GSH-reactive compounds (iodoacetamide (IACA), N-ethylmaleimide (NEM), diethyl maleate (DEM)) and compounds which need further metabolisation into GSH-reactive metabolites (cyclophosphamide (CP), 3-hydroxyacetanilide (3-HAA), 2-methylfurane (2-MF) [12]). Two pesticides (monuron (MN) and monulinuron (MLN)) were tested because their metabolite (4-chloroaniline) is known to generate hemogllobin adducts [29]. As the glutathione sulfhydryl group is more sensitive to alkylation than the hemoglobin sulfhydryl group [9], MN and MLN might also affect glutathione concentrations.

In incubations with $L A C A, N E M$ and DEM, reduced glutathione (GSH) was determined. The sum of both reduced and oxidized glutathione (= total glutathione (GT)) was determined when CP, 3-HAA, 2-MF, MN and MLN were tested. The 
loss of glutathione (GSH or GT depletion) as a consequence of the incubation with a test compound was used as a measure for the amount of GSH-reactive metabolite.

\section{Materials and Methods}

\section{Chemicals}

Reduced glutathione (GSH), Glucose-6-phosphate dehydrogenase (G6PDH; EC 1.1.1.49), glutathione reductase ( $\mathrm{EC}$ 1.6.4.2), $\beta$-glucuronidase/arylsulfatase (EC 3.2.1.31/EC 3.1.6.1), Glucose-6-phosphate (G6P), NADP, NADH, cyclophosphamide (CP; CAS: 6055-19-2) and 3-hydroxyacetanilide (3-HAA; CAS: 62142-1) were obtained from Sigma (St. Louis, USA). Diethyl maleate (DEM), N-ethylmaleimide (NEM) and iodoacetamide (IACA) were from Janssen Chimica (Beerse, Belgium). 2-Methylfurane (2-MF; CAS: 534-22-5) was from Merck (Darmstadt, Germany), and monuron (MN; CAS: 150-68-5) and monolinuron (MLN; CAS: 1746-81-2) were from Riedel-de Haën (Seelse, Germany). Collagenase B (from Clostridium histolyticum) was obtained from Boehringer (Mannheim, Germany). Water was demineralized and microfiltrated before use. All chemicals were of analytical grade quality.

\section{Animals}

Male Brown Norway rats (BN/M) of about 250 grams were used. Housing was as previously described [26]. When liver microsomes from rats pretreated with phenobarbital (PB microsomes) were used, phenobarbital ${ }^{T M}$ was added to the drinking water $\left(1 \mathrm{~g} \cdot \mathrm{l}^{-1}\right)$ during one week prior to the preparation of microsomes.

\section{Preparation of microsomal activating system and the isolation of hepatocytes}

The preparation of microsomal activating system was described previously [26]. The procedure for the isolation of hepatocytes was based on the methods described by Berry and Friend [4] and by Seglen [30]. A calcium and magnesium free buffer solution (100 $\mathrm{mM} \mathrm{NaCl}, 7 \mathrm{mM} \mathrm{KCl}, 39 \mathrm{mM}$ HEPES and $15 \mathrm{mM}$ $\mathrm{NaH}_{2} \mathrm{PO}_{4}$ ( $\mathrm{pH}$ adjusted to 7.4 with $\left.\mathrm{NaOH}\right)$ ) was perfused $\left(30 \mathrm{ml} \cdot \mathrm{min}^{-1}\right)$ in a single pass way through the isolated rat liver during 20 minutes. Next, a collagenase containing buffer ( $0.05 \mathrm{gram} \cdot 100 \mathrm{ml}^{-1}$ ), composed of $67 \mathrm{mM} \mathrm{NaCl}, 7 \mathrm{mM} \mathrm{KCl}$, $100 \mathrm{mM}$ HEPES and $5 \mathrm{mM} \mathrm{CaCl} \cdot 2 \mathrm{H}_{2} \mathrm{O}$ ( $\mathrm{pH}$ adjusted to 7.4 with $\mathrm{NaOH}$ ), was perfused in a recirculating way during 5 minutes. Hepatocytes were isolated from the liver, filtrated $(100 \mu \mathrm{M})$ and washed three times in a Krebs-Flenseleit buffer ( $\mathrm{pH} 7.4 ; 4$ minutes, centrifuge force: $25 \times \mathrm{g}$ ). The viability of the cells (more than $90 \%$ ) was determined with the Trypan-blue exclusion test [16]. 


\section{Preparation of erythrocytes and hemolysate}

Human blood was obtained from the Red Cross Blood Bank "Zuid Limburg", collected in sterile vacuum EDTA vessels, checked for viral infections, and kept overnight at $4^{\circ} \mathrm{C}$. Erythrocytes of at least 4 persons were pooled, washed three times using saline and diluted in $100 \mathrm{mM} \mathrm{Na} 2 \mathrm{HPO}_{4} / \mathrm{KH}_{2} \mathrm{PO}_{4}$ buffer (hemoglobin (Hb) concentration $100 \mathrm{~g} \cdot \mathrm{l}^{-1}, \mathrm{pH} 7.4$ ) in incubations with microsomes, or in Krebs-Henseleit buffer ( $\mathrm{Hb}$ concentration $27 \mathrm{~g}^{-1^{-1}}, \mathrm{pH} 7.4$ ) in incubations with hepatocytes.

Hemolysate was prepared from packed erythrocytes by dilution with ice-cold water (1:2). The cellular debris was removed by centrifugation ( 5 minutes, $2000 \times \mathrm{g}$ ).

\section{Incubations}

Each experiment consisted of incubations performed in triplicate. For direct GSHreactive compounds a single experiment was done. Experiments with CP, 3-HAA, 2-MF, MN and MLN and aqueous GSH solution, hemolysate and erythrocytes with or without microsomes were repeated three times on different days (total: $3 \times 3$ ). Co-incubation experiments of erythrocytes and hepatocytes were also repeated three times. Incubations with hepatocytes without erythrocytes, were based on a single experiment for CP, and repeated three times for 3-HA.A.

Incubations of direct GSH-reactive compounds with aqueous GSH or whole blood

Whole blood (1.5 ml) or an equal volume of aqueous GSH solution in phosphate buffered saline $(\mathrm{pH}=7.3)$, were incubated with the test compounds $(50 \mu \mathrm{l})$. IAcA and NEM were dissolved in phosphate buffered saline, and DEM in ethanol. Aqueous GSH solution (initial concentration $500 \mu \mathrm{M}$ ) was incubated with IAcA, NEM and DEM for 1 hour in a shaking waterbath (210 revolutions per minute) at $37^{\circ} \mathrm{C}$. Human blood (initial GSH concentrations 860,720 and $820 \mu \mathrm{M}$ ) was also incubated with IACA, NEM and DEM respectively $\left(37^{\circ} \mathrm{C}, 210\right.$ revolutions per minute). After an incubation period of 15 minutes, the GSH concentration was measured. Final concentrations of $0,0.5,1.0$ and $2.0 \mathrm{mM} \mathrm{IAcA}$, NEM and DEM were tested.

\section{Incubations with hemolysate}

To verify whether substances without direct activity to GSH (CP, 3-HAA, 2-MF, MN and MLN) were metabolically activated by erythrocytes themselves, GT depletion in hemolysate was tested (initial GT concentration 235 (standard error of the mean $(\mathrm{SE})=20 \mu \mathrm{M})$. After pre-incubating hemolysate for 5 minutes at $37^{\circ} \mathrm{C}$ in a shaking waterbath (80 revolutions per minute), $1 \mathrm{ml}$ test compound 
(final concentrations $0,0.5,1.0$ and $2.0 \mathrm{mM}$ ) was added. After 1 hour incubation the reaction was stopped by the addition of an equal volume of $5 \%(\mathrm{w} / \mathrm{v})$ trichloroacetic acid (TCA).

Co-incubations of aqueous GSH solution or erythrocytes with and without microsomal activating system

Erythrocytes final $\mathrm{Hb}$ concentration $23 \mathrm{~g} \cdot \mathrm{l}^{-1}$, final GT concentration 110 $(\mathrm{SE}=4) \mu \mathrm{M})$ or aqueous $\mathrm{GSH}$ solutions (final concentrations of $440(\mathrm{SE}=5) \mu \mathrm{M}$ were suspended in $100 \mathrm{mM} \mathrm{Na} \mathrm{HPO}_{4} / \mathrm{KH}_{2} \mathrm{PO}_{4}$ buffer (final volume $3 \mathrm{ml}$ ). For $2-\mathrm{MF}$ the initial $\mathrm{GT}$ concentration was $300(\mathrm{SE}=5) \mu \mathrm{M}$. Incubations were performed both with and without microsomal activating system. Both normal and PB microsomes were tested. The complete test system without test substance was pre-incubated for 5 minutes at $37^{\circ} \mathrm{C}$ in a shaking waterbath ( 80 revolutions per minute). Thereafter, $1 \mathrm{ml}$ test substance (final concentration between 0 and $2 \mathrm{mM}$ ) was added and incubated for 1 hour. For CP and 3-HAA, separate experiments were performed for test compound concentrations between 0 and $0.5 \mathrm{mM}$ and between 0.5 and $2.0 \mathrm{mM}$. After 1 hour incubation the samples were treated as previously described [26]. Finally, GT concentrations were measured.

\section{Incubations with hepatocytes}

Hepatocytes $\left(4.4 \cdot 10^{6}\right.$ cells $\left.\cdot \mathrm{ml}^{-1}\right)$ plus 3-HAA or CP solutions (final concentrations between 0 and $2.0 \mathrm{mM}$ ) were incubated in Krebs-Henseleit buffer ( $\mathrm{pH}$ 7.4) during 1 hour (incubation volume $1.5 \mathrm{ml}, 37^{\circ} \mathrm{C}$ ) in a shaking water bath ( 80 revolutions per minute). After the incubation period, $200 \mu \mathrm{l}$ TCA ( $8 \%$ ) was added to an equal volume of hepatocyte suspension and GT was determined. $100 \mu \mathrm{l}$ Hepatocytes were lysed with ice cold water $(1: 6.7 \mathrm{v} / \mathrm{v})$ and frozen until protein determination $\left(-70^{\circ} \mathrm{C}\right)$. The rest of the sample was centrifugated $(5 \mathrm{~min}, 25 \times \mathrm{g})$ and $1 \mathrm{ml}$ supernatant plus $200 \mu \mathrm{l} \mathrm{GSH}$ sollution (final concentration between 100 and $400 \mu \mathrm{M}$ ) was incubated again under the same conditions. In order to liberate possible conjugated metabolites, $\beta$-glucuronidase/arylsulfatase was added (final activity of $\left.650 \mathrm{U} \cdot \mathrm{ml}^{-1}\right)$. The pH was adjusted to 6.2 by addition of $175 \mu 13 \mathrm{M}$ $\mathrm{KH}_{2} \mathrm{PO}_{4}$.

\section{Co-incubations of erythrocytes and hepatocytes}

Co-incubations of erythrocytes and hepatocytes were performed in transwells ${ }^{T M}$ (Costar, Cambridge, England), that are composed of a well in which a smaller well (transwell) can be placed containing a porous membrane $(0.4 \mu \mathrm{m})$. Erythrocytes (2.6 ml, final $\mathrm{Hb}$ concentration $27 \mathrm{gl}^{-1}$ ) were pipetted into the well, and the hepatocytes $\left(1 \mathrm{ml}, 6.6 \cdot 10^{6}\right.$ cells $\left.\cdot \mathrm{ml}^{-1}\right)$ plus 3 -HAA $(0.5 \mathrm{mil}$, final concentration between 0 and $5 \mathrm{mM}$ ) were added to the transwell. After an incubation period of 
1 hour, the erythrocytes were pipetted into plastic vials and packed by centrifugation. The rest of the procedure was the same as in co-incubations of microsomes and erythrocytes. Finally, GT concentrations were determined.

\section{Analytical procedures}

GSH was determined, essentially as described by Anderson [2]. Sample preparation was described previously [9]. GT in the TCA supernatants was determined after dilution ( 9 fold) with buffer by the cyclic oxidation reduction method described by Anderson [2]. Lactate dehydrogenase (LDH) activity was determined using NADH consumption during pyruvate transformation to lactate [24]. Microsomal and hepatocyte protein content was determined according to Lowry et al. [20]. Hemoglobin concentrations were determined by measuring cyanmethemoglobin according to Van Kampen and Zijlstra, [17].

\section{Statistical analysis}

GT depletion caused by CP, 3-HAA, 2-MF, MN and MLN was analyzed separately for both incubations with normal and $\mathrm{PB}$ microsomes by linear regression, using a model including day of experiment and concentration as explanatory variables. Using that model we tested whether slopes of GT depletion versus concentration were significant for each of the metabolizing systems, correcting for the day of the experiment.

\section{Results}

\section{Incubations without addition of metabolic activating system}

Depletion of GSH by IACA, NEM and DEM or depletion of GT by CP, 3-HAA and $2-\mathrm{MF}$ in incubations without metabolic activating system (MAS), is shown in Figures $3.2,3.3$ and 3.4 for aqueous GSH solutions, erythrocytes and hemolysate
respectively.

In incubations with aqueous GSH solution (initial GSH concentration $500 \mu \mathrm{M}$ ), GSH was depleted by the direct GSH-reactive compounds IACA, NEM and DEM. NEM was the most reactive compound as GSH was almost fully depleted when $0.5 \mathrm{mM}$ NEM was tested, followed by IAcA which was almost fully depleted at a concentration of $1.0 \mathrm{mM}$. DEM was the least reactive compound.

The incubations with erythrocytes and IACA, NEM and DEM showed that erythrocyte GSH could also be depleted by these compounds, indicating that these GSH-reactive electrophiles could pass the erythrocyte membrane. IACA was the 


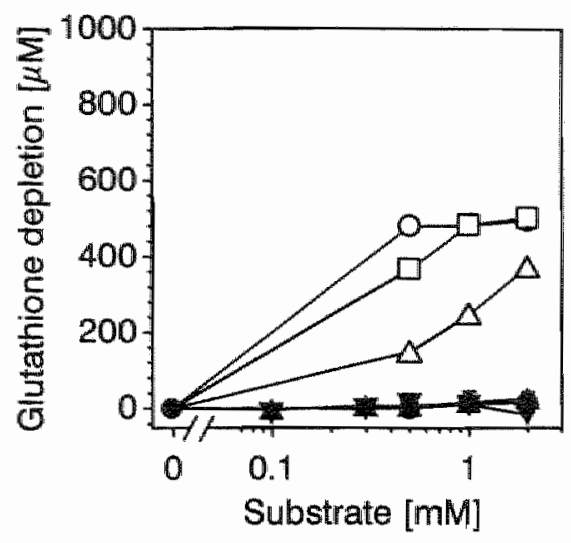

Figure 3.2: Incubations with aqueous GSH solution. GSH depletion in incubations with IACA $\square$, NEM $O$ and DEM $\triangle$ and GT depletions in incubations with CP A, 3-HAA and 2-MF are shown. The mean of the GSH or GT depletion plus the SE of the GT depletion is shown (interexperimental variation). For some of these incubations the SE falls within the datapoints.

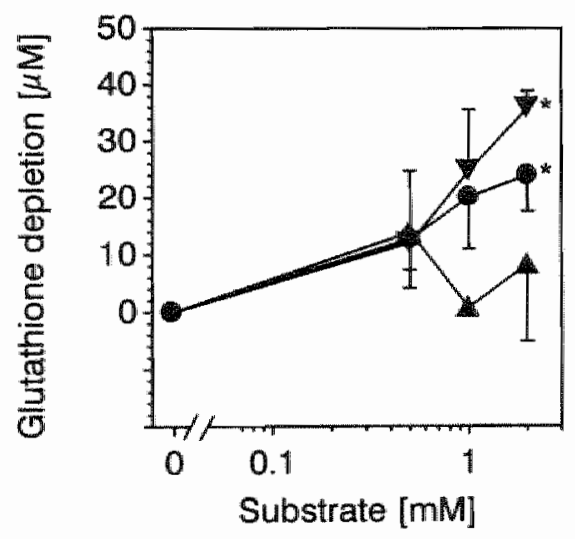

Figure 3.4: Incubations with hemolysate. GT depletions in incubations with CP A, 3-HAA and 2-MF are shown. The mean and the SE of the GT depletion is shown (interexperimental variation). The GT depletion was concentration dependent both for 3-HAA and 2MF (" $p \leq 0.01$ in both cases).

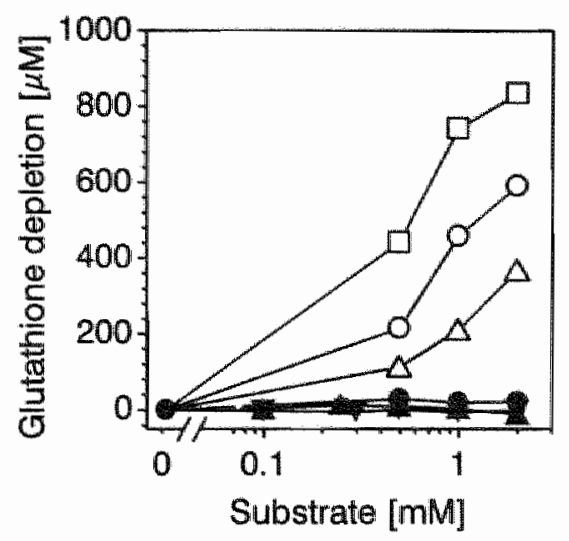

Figure 3.3: Incubations with erythrocytes. GSH depletion in incubationss with IACA $\square$, NEM $\square$ and DEM $\triangle$ and GT depletions in incubations with CP $\mathbf{A}, 3$ HAA and 2-MF are shown. The mean of the GSH or GT depletion plus the SE of the GT depletion is shown (interexperimental variation). For some of these incubations the SE falls within the datapointis. 


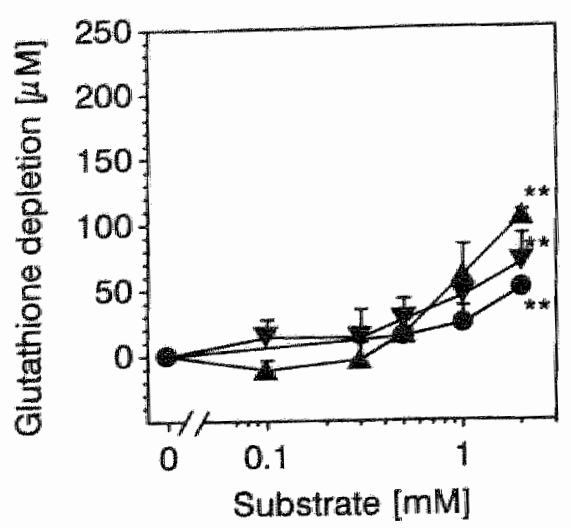

Figure 3.5: Incubations with aqueous GSH solution and microsomes of untreated rats. GT depletions in incubar tions with CP $4,3-H A A$ and $2-M F$ - are shown. The mean and the $\mathrm{SE}$ of the GT depletion is shown (interexperimental wariation].. The GT depletion was concentration dependent for all compounds bested (" $p \leq 0.001$ in all cases).

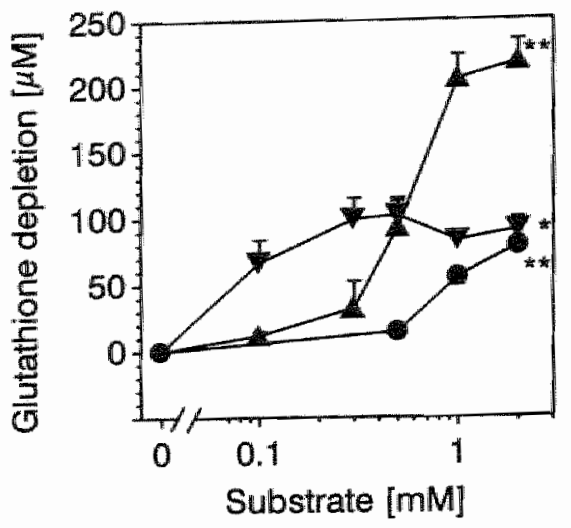

Figure 3.6: Incubations with aqueots GSH solution and microsomes of PB pretreated rats. The mean and the SE (interexperimental variation) of the GT depletions in incubations with CP $A, 3-\mathrm{HAA}$ F and 2-MF are shown. In some cases the SE falls within the datapoints. The GT depletion was concentration dependent for all compounds ( ${ }^{*} p \leq 0.01$ for $3-\mathrm{HAA}^{* * *} p \leq 0.001$ for $\mathrm{CP}$ and $\left.2-\mathrm{MF}\right)$.

most reactive compound in these incubations as $97 \%$ of the initial GSH concentration was depleted at a IACA concentration of $2 \mathrm{mM}$, followed by NEM and DEM ( $82 \%$ and $45 \%$ resp ; $2 \mathrm{mM}$ test compound).

GT was not depleted in incubations with aqueous GSH solution or erythrocytes when CP, 3-HAA, 2-MF, MN and MLN were tested.

In incubations with hemolysate, 3-HAA and 2-MF caused a concentration dependent GT depletion ( $p \leq 0.01$ in both cases). The amount of GT depleted was

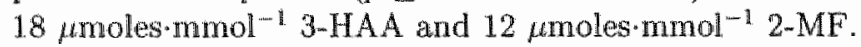

\section{Incubations with microsomal activating system}

In incubations with aqueous GSH solution and normal microsomes (Figure 3.5), GT was depleted in a concentration dependent way by CP, 3-HAA and 2-MF ( $p \leq$ $0.001 \mathrm{in}$ all cases $)$. The amount of $\mathrm{GT}$ depleted by $\mathrm{CP}$ was $48 \mu \mathrm{moles} \cdot \mathrm{mmol}^{-1} \mathrm{CP}$, by 3 -HAA was $36 \mu$ moles mmol $^{-1} 3-\mathrm{HAA}$ and by $2-\mathrm{MF}^{2}$ was $25 \mu$ moles $\cdot \mathrm{mmol}^{-1}$ $2-\mathrm{MF}$.

When CP, 3-HAA and 2-MF were tested in incubations with aqueous GSH solution and $P B$ microsomes (Figure 3.6), higher GT depletions were found compared 


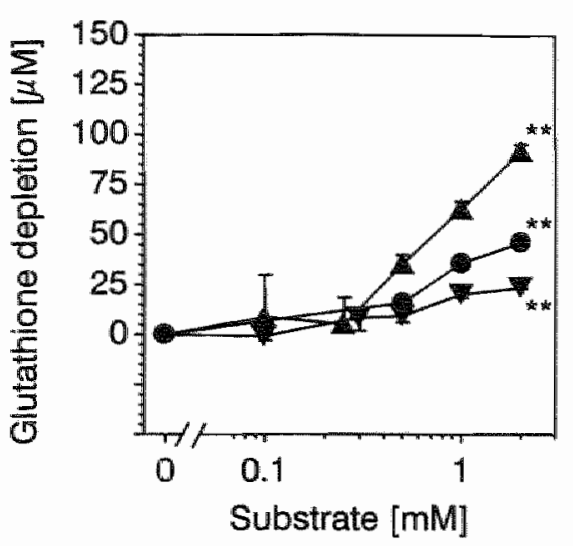

Figure 3.7: Incubations with erythrocytes and microsomes of untreated rats. GT depletions in incubations with $\mathrm{CP}$ A, 3-HAA $\nabla$ and 2-MF are shown. The mean and SE of the GT depletion is shown (interexperimental variation). For some of these incubations the SE falls within the datapoints. The GT depletion was concentration dependent for all compounds tested (** $p \leq 0.001)$.

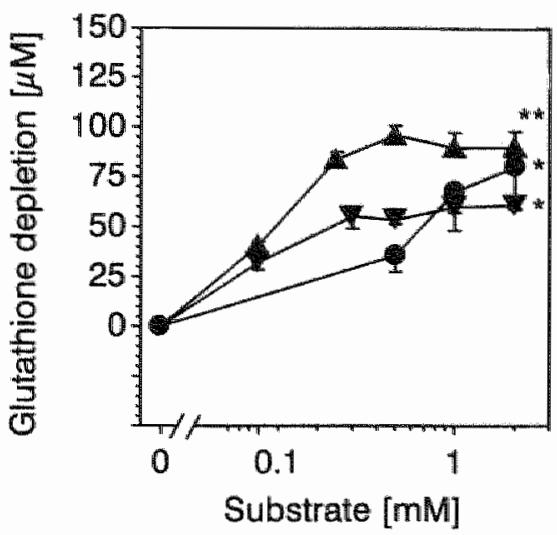

Figure 3.8: Incubations with erythrocytes and microsomes of $\mathrm{PB}$ pretreated rats. GT depletions in incubations with CP 4 3-HAA and 2-MF are shown. The mean and SE of the GT depletion is shown (interexperimental variation). The $\mathrm{GT}$ depletion was concentration dependent for all compounds tested $\left({ }^{*} p \leq 0.01\right.$ for $3-\mathrm{HAA}$ and $2-\mathrm{MF}_{\mathrm{i}} *{ }^{*} p \leq 0.001$ for CP).

to incubations with normal microsomes. GT depletions were concentration dependent for all three compounds tested ( $p \leq 0.01$ for 3 -HAA; $p \leq 0.001$ for CP and 2-MF). The amount of GT depleted by CP and was $92 \mu$ moles mmol $^{-1} \mathrm{CP}$, and by $2-\mathrm{MF} 41 \mu$ moles $\mathrm{mmol}^{-1} 2-\mathrm{MF}$. For 3-HAA, the amount of GSH-reactive metabolite formed was maximal at a 3 HAA concentration of $0.3 \mathrm{mM}$. At this 3-HAA concentration the initial GSH concentration was about $25 \%$ depleted.

GT was not depleted when MN or MLN were tested in incubations with aqueous GSH solution, irrespective of the kind of microsomes used.

In incubations with erythrocytes and normal microsomes (Figure 3.7), GT wass depleted in a concentration dependent way by CP, 3-HAA and 2-MF $(p \leq$ 0.001 in all cases). The amount of GT depleted in incubations with CP was $46 \mu$ moles $\mathrm{mmol}^{-1} \mathrm{CP}$, with 3 -HAA was $11 \mu$ moles mmol ${ }^{-1}$ 3-HAA, and with 2-MF $23 \mu \mathrm{moles} \cdot \mathrm{mmol}^{-1} 2-\mathrm{MF}$.

In incubations with erythrocytes and $P B$ microsomes (Figure 3.8), G $\mathrm{C}$ was also depleted in a concentration dependent way when CP, 3-HAA and 2-MF were tested $\left(p \leq 0.01\right.$ for $3-$ HAA and $2-\mathrm{MF}_{;} p \leq 0.001$ for $\mathrm{CP}$ ). Considering $\mathrm{CP}$, all glutathione was consumed at $0.5 \mathrm{mM}$ CP. The amount of GT depleted in incubations with $\mathrm{CP}$ was $188 \mu$ moles $\mathrm{mmol}^{-1} \mathrm{CP}$ regarding $\mathrm{CP}$ concentrations 
up to $0.5 \mathrm{mM}$. 3-HAA caused a GT depletion of 30 umolles $\mathrm{mmol}^{-1}$ 3-HAA and

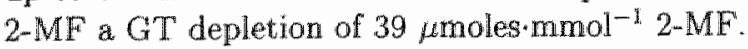

Again, GT was not depleted when MN or MLN were tested in incubations with humari erythrocytes, irrespective of the kind of microsomes used.

GT was only depleted in incubations with aqueous GSH solution or erythrocytes when both microsomes (normal or PB) and NADPH regenerating system were added.

\section{Incubations with hepatocytes}

viability of hepatorytes

The viability of hepatocytes was tested by measurement of $\mathrm{LDH}$ leakage into the medium [16]. LDH leakage was low during the first 50 minutes of the incubation period and increased thereafter (data not shown). The wiability of the hepatocytes was not affected by 3-HAA or CP (final concentration $2 \mathrm{mM}$ ) as the LDH activity in the supernatant was not higher than in controls after a one-hour incubation period (Table 3.1). Incubation of hepatocytes with DEM (final concentration $1 \mathrm{mM}, 15$ minutes, $37^{\circ} \mathrm{C}$ ) depleted the GT concentration up to $72 \%$ but had no effect on LDH leakage (data not shown).

Table 3.1: Viability of hepatocytes, hepatocyte GT depletion after incubation with 3-HAA and CP and excretion of GSH-reactive metabolites by hepatocytes.

\begin{tabular}{cc||ccc}
\hline test compound & $\begin{array}{c}\text { concentration } \\
(\mathrm{mM})\end{array}$ & $\begin{array}{c}\mathrm{LDH} \\
\left(\mathrm{U} \cdot \mathrm{I}^{-1}\right)\end{array}$ & $\begin{array}{c}\text { GT-hep } \\
\left(\mu \mathrm{moles} \cdot \mathrm{g}^{-1}\right.\end{array}$ & $\begin{array}{c}\text { GT-sup } \\
(\mu \mathrm{M})\end{array}$ \\
\hline 3-HAA & 0 & 1616 & $39(\mathrm{SE}=5)$ & 369 \\
& 1.0 & 1564 & $\mathrm{n} . \mathrm{d}$. & 319 \\
& 2.0 & 1545 & $32(\mathrm{SE}=3)$ & 294 \\
& 0 & & 38 & 141 \\
$\mathrm{CP}$ & 1.0 & 1616 & $\mathrm{n} \cdot \mathrm{d}$. & $\mathrm{n} . \mathrm{d}$. \\
& 2.0 & 1494 & 30 & 135 \\
\hline
\end{tabular}

LDH is the LDH activity $\left(\mathrm{U}^{-1} \mathrm{I}^{-1}\right)$ in the incubation medium after a 1-hr incubation period with 3-HAA and CP; GT-hep is the mount of GT in hepatocytes per gram protein $\left(4 \mathrm{~g} \cdot \mathrm{I}^{-1}\right)$, after a 1 -hr incubation period with $3-\mathrm{HAA}$ and CP; GT-sup is the $\mathrm{G} T$ concentration in supernatants of incubations with hepatocytes and 3-HAA and CP, which were incubated again with aqueous GSH solution for 1 hour. 


\section{Incubations with hepatocytes without erythrocytes}

GT depletion in hepatocytes (GT-hep; Table 3.1) was $7 \mu$ moles. $\mathrm{g}^{-1}$ protein when $2 \mathrm{mM} 3-\mathrm{HAA}$ was tested and $8 \mu$ moles $\mathrm{g}^{-1}$ protein when $2 \mathrm{mM} \mathrm{CP}$ was tested. The decrease in hepatocyte GT concentration caused by 3-HAA was concentration dependent $(p=0.01)$.

The ability of GSH-reactive metabolites to pass the hepatocyte membrane was tested. For this purpose, after incubation of substrate with hepatocytes, the intact cells were removed by centrifugation and the supernatant was incubated again with GSH during one hour under the same conditions. Table 3.1 shows the results of a typical experiment both for 3-HAA and CP. It shows that the GT concentration in the supernatants (GT-sup) decreased as a result of the former incubation of hepatocytes with the test compound, indicating that GSH-reactive 3-HAA and CP metabolites are excreted by the hepatocytes.

Electrophiles generated by hepatocytes may also be detoxified by conjugation in the hepatocyte and excreted into the medium. The presence of glucuronide conjugates was tested by means of the addition of $\beta$-glucuronidase/arylsulfatase to the medium of the second (GSH containing) incubation, so that GSH may be able to react with the released metabolite. However, addition of $\beta$ glucuronidase/arylsulfatase did not lead to more GT depletion.

\section{Co-incubations of erythrocytes and hepatocytes}

Erythrocyte GT concentration was not affected when erythrocytes and hepatocytes were co-incubated. Addition of 3-HAA to these incubations (final concentrations up to $5 \mathrm{mM}$ ) did not affect erythrocyte GT, neither when normal hepatocytes were used nor when hepatocytes from phenobarbital pretreated rats were tested.

Pre-incubation of hepatocytes with DEM, which lead to a $72 \%$ GT depletion. in the hepatocytes, did also not affect the erythrocyte GT concentration. As electrophiles may be sulfated in the hepatocyte, hepatocytes were isolated and incubated with sulfate-free buffer. Incubation in sulfate-free medium is known to affect sulfation capacity [8]. However, no effect on erythrocyte GT was found.

\section{Discussion}

Most cell types are more or less able to (de)toxify foreign chemicals. The hepatocyte is known as a metabolically very active cell and erythrocytes have some metabolic activity also [3]. Foreign compounds, toxified by hepatocytes by means of cytochrome P-450 activity, may be detoxified by phase II metabolism (e.g. glucuronidation, sulfation, glutathione conjugation). This may lead to 
a shortage of hepatocyte phase II conjugation factors like UDP-glucuronic acid, 3'-phosphoadenosine-5's phosphosulfate and reduced glutathione (GSH). Conjugated and/or unconjugated metabolites may be released by the hepatocyte into the sinusoids. This means that erythrocytes can be exposed to these metabolites. When the metabolites are able to pass the erythrocyte membrane, the biochemical functions of the erythrocyte may be affected.

In the present investigation, we developed a set of in vitro test-systems (Figure 3.1), that may gain an insight into the interplay between the bio-activation and inactivation functions of liver and blood, using glutathione depletion as dependent variable. A comparable method was described by Tingle and Park [34], who found methemoglobin formation in human erythrocytes when dapsone was tested in incubations with rat liver microsomes and human erythrocytes. To approach the physiological in vivo situation more closely, we tested whether hepatocytes are able to generate GSH-reactive metabolites from CP or 3-HAA, and the ability of 3-HAA metabolites to deplete erythrocyte GT. As a large variety of variables (beside glutathione depletion) can be tested, the test-systems may extend the current set of techniques. GSH is an efficient scavenger of reactive electrophilic substances, especially in combination with glutathione dependent enzymes $[6,21,22,31]$. Because of this property, a decrease of the GSH concentration (glutathione depletion) causea by the test compound, has been used as a measure for the amount of GSH-reactive compounds in the incubate $[11,12,13]$.

IACA, NEM and DEM are direct GSH-reactive compounds which need no metabolic activation [9]. In incubations with aqueous GSH solution and in incubations with erythrocytes, GSH was depleted. This means that these test compounds are able to pass the erythrocyte membrane and deplete erythrocyte GSH. As GSH was also depleted in incubations with the GST lacking aqueous GSH solution, catalysation of the conjugation reaction by erythrocyte GST is not needed for these highly reactive compounds. CP, 3-HAA, 2-MF, MN and MLN did not influence GT concentrations (GT is the sum of both reduced and oxidized glutathione) of aqueous GSH solutions or erythrocytes when no external metabolizing system was added, indicating that these compounds have no direct action on GSH.

Both 3-HAA and 2-MF depleted GT in hemolysate but not in incubations with aqueous GSH solution or erythrocytes. The results indicate that a factor in the hemolysate is capable to metabolize 3-HAA and 2-MF resulting in GSH conjugation. This metabolic activity was not explained by mere GST catalyzed glutathione conjugation to the parent compounds as addition of glutathione S-transferase $\pi$ $\left(3.2 \mathrm{U} \cdot \mathrm{ml}^{-1}\right)$ to the incubations with aqueous GSH solution and 3-HAA or 2-MF, bad no effect on the GT concentration (data not shown).

Only when rat MAS was added to incubations of CP, 3-HAA or 2-MF and aqueous GSH solution or erythrocytes, GT was depleted. GT depletions were concentration dependent when normal or PB microsomes were used. Addition of NADPH 
was a requirement. This indicates that metabolites of CP, 3-HAA and 2-MF are generated by MAS and that these metabolites are able to enter the erythrocyte. For all three compounds tested, GT depletions were higher in incubations with aqueous GSH solution than in erythrocytes when normal microsomes were tested. This may indicate that the erythrocyte membrane acts as a barrier for metabolites of $\mathrm{CP}, 3-\mathrm{HAA}$ and 2-MF, generated by microsomes. If the erythrocyte membrane would limit diffusion to less than $80 \mathrm{nmoles} \cdot \mathrm{h}^{-1} \cdot \mathrm{m}^{-2}$ membrane, GT depletion would be less than $10 \%$ during the assay period and therefore probably not be detectable. (In $200 \mu \mathrm{l}$ erythrocytes the amount of GSH is 300 nanomoles. The amount of GSH-reactive metabolite needed to deplete $10 \%$ of this GSH pool would be 30 nanomoles. The membrane surface of $200 \mu l$ erythrocytes is $0.36 \mathrm{~m}^{2}[15]$ ). Both in aqueous GSH solutions and in erythrocytes, GT depletion by $\mathrm{CP}, 3-\mathrm{HAA}$ and 2-MF was higher with $\mathrm{PB}$ pretreated microsomes than with normal microsomes. This means that the metabolite generation of $C P$, 3-HAA and 2-MF is mediated by the phenobarbital inducable MAS. When MN or MLN were tested in incubations with aqueous GSH solution or erythrocytes, the quantity of reactive metabolites formed in the present study was insufficient. to give a detectable GT depletion. Addition of normal or PB microsomes had no influence. MN and MLN are known to give (4-chloroanilin) Hb adducts in rats [29]. Presence of reactive MN or MLN metabolites in erythrocytes would not only lead to hemoglobin adducts but also to glutathione conjugation, as GSH is more reactive than the nucleophilic sites in $\mathrm{Hb}$ [9].

Hepatocyte GT decreased $7-8 \mu$ molles $\mathrm{g}^{-1}$ protein in incubations with $2 \mathrm{mM} \mathrm{CP}$ or 3-HAA. The protein concentration of hepatocytes is about $200 \mathrm{~g} \cdot 1^{-1}$, which means that the GT depletion in hepatocytes is about $1.5 \mathrm{mM}$ at $2 \mathrm{mM} \mathrm{CP}$ or 3-HAA. To verify whether a substantial fraction of the metabolites formed was excreted by the hepatocytes, the supernatants of the 3-HAA or CP hepatocyte incubations were reincubated with GSH during one hour under the same conditons. The GT depletion in the supernatant of incubations with 3-HAA (2 mM) was $75 \mu \mathrm{M}$. When the volumes of hepatocytes and supernatants $(22 \mu \mathrm{l}$ and $1.5 \mathrm{ml}$ respectively) are taken into account, the total amount of GSH-reactive 3-HAA metabolite within the cells and excreted into the supernatant can be calculated to be 33 and 113 nanomoles respectively, when we assume that the metabolites are equally distributed over hepatocytes and supernatant. These results indicate that a substantial fraction (77\%) of the GSH-reactive 3-HAA metabolites formed by the hepatocytes was excreted. For CP $(2 \mathrm{mM})$, only $21 \%$ of the GSH-reactive metabolites was excreted by the hepatocytes (GT depletion in the supernatant $=6 \mu \mathrm{M}$ ). For this reason 3-HAA was used in co-incubations of hepatocytes and erythrocytes. For technical reasons enforced by the geometry of the "transwells", the excreted metabolite concentration was diluted even further in the co-incubations of hepatocytes and erythrocytes (incubation volume $4.1 \mathrm{ml}$ instead of $1.5 \mathrm{ml})$, leading to a calculated 3-HAA metabolite concentration of 
$28 \mu \mathrm{M}$ outside the erythrocytes at the end of the incubation. If we suppose that all of this substance enters the erythrocyte during the experiment, a detectable depletion of about $30 \%$ would have occurred. However, in co-incubations of he patocytes and erythrocytes no effect of $3-\mathrm{HAA}$ on erythrocyte GT was found. In practice uptake limitation may therefore be an important factor. Assuming that metabolite excretion of the hepatocytes is linear with time, the mean metabolite concentration outside the erythrocytes was $14 \mu \mathrm{M}$. Using Fick's law we can calculate that the needed mass transport ( $80 \mathrm{nmoles} \cdot \mathrm{h}^{-1}$ "m ${ }^{-2}$, see above) can only be reached with a permeability caefficient of at least $0.7 \cdot 10^{-8} \mathrm{~cm} \cdot \mathrm{s}^{-1}$. For comparison, known values are about $10^{-7} \mathrm{~cm} \cdot \mathrm{s}^{-1}$ for glucose and $10^{-10} \mathrm{~cm} \cdot \mathrm{s}^{-1}$ and lower for ions [1]. This indicates that an effective depletion would only be detectable within one hour when the diffusion uptake speed of the metabolites was at lleast about $10 \%$ of the uptake speed of glucose.

To increase the excretion of GSH-reactive metabolites by the hepatocytes, hepatocytes were pre-incubated with diethyl maleate in order to deplete their glutathione store. While this treatment lead to over $70 \%$ depletion of GT in the hepatocytes, no effect of this treatment on erythrocyte GT was found. Rashed et al. described that 3-HAA and 3-HAA metabolites generated by oxidative metabolism, are sulfated and glucuronidated in mice [28]. In their study urinary glucuronides and sulfates of 3.HAA and its metabolites accounted for $80 \%$ of the administered dose. Since sulfation by isolated hepatocytes can extensively be reduced when no inorganic sulfate is administered [8], this treatment may increase the amount of unconjugated reactive metabolites in the hepatocytes and, in case of excretion, in the supernatant. However, isolation of hepatocytes with sulfate-free buffer solutions, and incubation of hepatocytes and erythrocytes in sulfate-free Krebs buffer, did not deplete erythrocyte GT when 3-HAA was tested. Addition of $\beta$-glucuronidase/arylsulfatase to incubations composed of GSH and supernatant which was previously incubated with hepatocytes and $3-\mathrm{HAA}$, had also no influence on GT depletions.

In summary, the direct GSH-reactive compounds IAcA, NEM and DEM were able to enter the erythrocyte and deplete the erythrocyte GSH store. CP, 3-HAA and $2-\mathrm{MF}$ only depleted erythrocyte GT when MAS was added indicating that GSHreactive metabolites were formed which were able to enter the erythrocyte. Hepatocytes also metabolised 3-HAA and CP into GSH-reactive metabolites. They were able to excrete a fraction of these GSH-reactive metabolites into the supernatant. For 3-HAA, no erythrocyte GT depletion was found in co-incubations of hepatocytes and erythrocytes which may be explained by uptake limitation of the GSH-reactive metabolites into the erythrocytes.

Acknowledgements We wish to thank Prof Dr P.Th. Henderson for critical reading of the manuscript, Dr A. Kester Lor his advice in the statistical analyses and the Red Cross Blood Bank "Zuid Limburg ${ }^{*}$ for collecting the blood samples. 


\section{Bibliography}

[1] Alberts, B., Bray, D., Lewis, J., Raf, M., Roberts, K. and Watson, I.D. Molecular biology of the cell, Garland Publishing Inc., New York, 1983, p. 287.

[2] Anderson, M.E. Determination of glutathione and glutathione disulfide in biological samples, in: Meister, A. (ed.), Methods Enzymol., 1985, 113, pp. 548-555.

[3] Belvedere, G. and Tursi, F. (1981) Styrene oxidation to styrene oxide in human blood erythrocytes and lymphocytes. Res. Commun. Chem. Pathol. Pharmacol. $33,273-282$.

[4] Berry, M.N. and Friend, D.S. (1969) High yield preparation of isolated rat liver parenchymal cells. J. Cell Biol. 43,506-520.

[5] Calabrese, E.J., Tilli, F., Itorton, H.M. and Stoddard, A. (1988) The effects of ethanol on the hematotoxicity of twelve pharmaceutical and environmental agents. J. Environ. Science Health A23, 359-367.

[6] Deneke, S.M. and Fanburg, B.L. (1989) Regulation of cellular glutathione. Am. J. Physiol. 257, L163-L173.

[7] Duverger-van Bogaert, M., Crutzen-Fayt, M.C. and Stecca, C. (1991) Mutagenicity of some heterocyclic amines in Salmonella typhimurium with metabolic activation by human red blood cell cytosol. Mutat. Res. 261, 261-265.

[8] Evelo, C.T.A., Versteegh, J.F.M. and Blaauboer, B.J. (1984) Kinetics of the formation and secretion of the aniline metabolite 4-aminophenol and its conjugates by isolated rat hepatocytes. Xenobiotica 1.4, 409-416.

[9] Evelo, C.T.A. and Henderson, P.Th. (1988) Influence of glutathione on the formation of cysteine alkylation products in human hemoglobin. Toxicology $52,177-186$.

[10] Fazi, A., Mancini, U., Piatti, E., Accorsi, A. and Magnani, M. (1991) Human red blood cells as bioreactors for the inactivation of harmful xenobiotics. Biotechnol. Appl. Biochem. 14,60-68.

[11] Garle, M.J., Khan, J. and Fry, J.R. (1988) Depletion of glutathione by the hepatotoxins paracetamol and bromobenzene, and their non-hepatotoxic analogues, in a fortified liver microsomal system. Toxicol. Vitro $2,247-252$.

[12] Garle, M.J. and Fry, J.R. (1989) Detection of reactive metabolites in vitro. Toxicology $54,101-110$.

[13] Garle, M.x, and Fry, J.R. (1990) Reactive netabolite formation catalysed by cytochrome P-450j. Toxicol. Vitro 4, 493-496.

[14] Guengerich, F.P. and Liebler, D.C. (1985) Enzymatic activation of chemicals to toxic metabolites. Crit. Rev. Toxicol. 14, 259-307.

[15] Guyton, A.C. Textbook of medical physiology, WB Saunders Company, Philadelphia, 1986.

[16] Jauregui, H.O., Hayner, N.T., Driscoll, J.L., Williams-Holland, R, Lipski, M.H. and Galletti, P.M. (1981) Trypan blue dye uptake and lactate dehycrogenase in adult rat hepatocytes-freshly isolated cells, cell suspensions, and primary monolayer cultures. In Vitro Cell Diev. Biol. 17, 1100-1110.

[17] van Kampen, E.J. and Zijlstra, W.G. (1965) Determination of hemoglobin and its derivates. Adv. Clin. Chem. 8, 141-187.

[18] Ketterer, B. (1982) The role of nonenzymatic reactions of glutathione in xenobiotic metabolism. Drug metabol. Rev. 13, 161-187. 
[19] Lo Jacono, F., Stecca, C. and Duverger, M. (1992) Mutagenic activation of benzola]pyrene by human red blood cells. Mutat. Res. 268, 21-26.

[20] Lowry, O.H., Rosebrough, N.J., Farr, A.L. and Randall, R.J. (1951) Protein measurement with the Folin phenol reagent. J. Biol. Chem. 193, 265-275.

[21] Mannerwik, B. atid Danielson, U.H. (1988) Glutathione transferases: structure and catalytic activity. Crit. Rev. Biochem. 23, 283-337.

[22] Meister, A. and Anderson, M.E. (1983) Glutathione. Annu. Rev. Biochem. 52, 711-760.

[23] Mieyal, J.J., Ackerman, R.S., Blumer, J.L. and Freeman, L.S. (1976) Characterization of enzyme-like activity of human hemoglobin; Properties of the hemoglobin-P450 reductasemcoupled aniline hydroxylase systern. J. Biol. Chem. 251, 3436-3441.

[24] Moss, D.W. Henderson, A.R. and Kachmar, J.F. Enzymes, in: Tietz, N.W. (ed.) Textbook of clinical chemistry, vol. 1, W.B. Saunders Company, Philadelphia, 1986, p. 1532.

[25] Norppa, H., Vainio, H. and Sorsa, M. (1983) Metabolic activation of styrene by erythrocytes detected as increased sister chromatid exchanges in cultured human lymphocytes. Cancer Res. 43, 3579-3582.

[26] Palmen, N.G.M. and Evelo, C.T.A. (1993) Glutathione depletion in human erythrocytes as an indicator for microsomall activation of Cyclophosphamide and 3-Hydroxyacetanilide. Toxicology 84, 157-170.

[27] Plaa, G.L. Toxic responses of the liver, in: Klaassen, C.D., Amdur, M.O. and Doull, J. (eds.) Toxicology; the basic science of poisons, vol. 3 , Macmillan publishing company, New York, 1986, p. 286-309.

[28] M.S. Rashed, T.G. Myers and S.D. Nelson, Hepatic protein arylation, glutathione depletion, and metabolite profiles of actaminophen and a non-hepatotoxic regioisomer, 3'-hydroxyacetanilide, in the mouse. Drug Metab. Dispos., 18 (1990) 765-770.

[29] Sabbioni, G. and Neumann, H.G. (1990) Biomonitoring of arylamines: hemoglobin adducts of urea and carbamate pesticides. Carcinogenesis 11, 111-115.

[30] Seglen, P.O. (1973) Preparation of rat liver cells. Exp. Cell Res. 82, 391-398.

[31] Sies, H. (1986) Biochemistry of oxidative stress. Angew. Chem. Int. Ed. Engl. 25, 1058-1071.

[32] Stecca, C. and Duverger-van Bogaert, M. (1989) N-Demethylation reactions in intact erythrocytes and erythrocyte supernatant. Arch. Toxicol. Suppl 13, 291293.

[33] Strange, R.C., Johnson, P.H., Lawton, A., Moult, J.A., Tector, M.J., Tyminski, R.J. and Cottion, W. (1982) Clin. Chim. Acta 120, 251-260.

[34] Tingle, M.D. and Park, B.K. (1993) The use of a three compartment in vitro model to investigate the role of hepatic metabolism in drug-induced blood dyscrasias. Br. J. Clin. Pharmaceutics 36, 31-38.

[35] Tursi, F., Samaia, M., Salmona, M. and Belvedere, G. (1983) Styrene oxidation to styrene oxide in human erythrocytes is catalyzed by oxyhemoglobin. Experientia $39,593-594$ 
Chapter 4

\section{Toxicokinetics of dimethylacetamide (DMAc) in rat isolated perfused liver}

Palmen ${ }^{1}$, N.G.M., Evelo', C.T.A., Borm ${ }^{2}$, P.J.A. and Henderson ${ }^{3}$, P.Th.

1 Department of Pharmacology, Section Toxicology

2 Department of Health Risk Analysis

University of Limburg, PO Box 616,6200 MD Maastricht

3 TNO Medical Biological Laboratory, PO Box 5815, 2280 HV Rijswijk, The Netherlands.

Published in: Human and Experimental Toxicology 12 (1993) 127-133. 


\section{Summary}

Dimethylacetamide (DMAc) is a skin-penetrating solvent able to induce hepatic damage after chronic exposure. Previous research has indicated that metabolism may be saturated at its present TLV/TWA (10 ppm). Biological monitoring of monomethylacetamide (MMAc), the primary metabolite of DMAC, might therefore underestimate exposure to DMAC and related health hazards. We used the recirculating perfusion technique in isolated rat liver to evaluate DMAc metabolism. Medium concentrations starting at about $30,50,100$ and $275 \mu \mathrm{M}$, respectively, were tested. Perfusate samples were taken regularly and analyzed for DMAc; pharmacokinetic parameters (extraction ratio and clearance) were calculated for exch perfusion. Inlet DMAc concentrations were calculated and concentration groups divided in $16,36,70,160,225 \mu \mathrm{M}$. The extraction ratio of the $16 \mu \mathrm{M}$ group differed significantly from the other concentrar tion groups tested. DMAc metabolism was saturated at a DMAc concentration of $36 \mu \mathrm{M}$. Extraction ratios were unaffected when cimetidine, an inhibitor of cytochrome P-450 activity, was added to the perfusion medium or when cimetidine-pretreated animals were used. DMAc clearance was $2.20 \mathrm{mll} \mathrm{min}^{-1}$ at a medium concentration of about $36 \mu \mathrm{M}$. Extrapolation of the observed (rat)livex clearance to man showed that airborne concentrations of $18 \mathrm{ppm}$ would, under the presumptions used, lead to saturated metabolism of DMAc; however, saturation at even lower concentrations could not be excluded.

\section{Introduction}

Dimethylacetamide (DMAc) is a colourless solvent which is used in the production of synthetic fibres, plastics, resins and gums and as an adjuvant for veterinary purposes. Its acute toxicity is low, with an oral $L D_{50}$ of $4 \mathrm{~g}^{\mathrm{kg}} \mathrm{kg}^{-1}$ in the rat [6]. The primary effects occurring at high dosage are respiratory failure and atonic state of the muscles. The most important effect considered for standard setting is the occurrence of hepatotoxicity at relativily low chronic exposures $[7,10]$. DMAc's liver toxicity might be related to specific biotransformation into (intermediate) reactive metabolites such as $\mathrm{N}$-hydroxymethyl $\mathrm{N}$-methylacetamide which was detected in urine of rats treated subcutaneously with ${ }^{14} \mathrm{C}$ labeled DMAc [16]. In humans the only metabolite observed in vivo was monomethylacetamide (MMAc) $[5,11,13]$. However, data with regard to the extent of its metabolism are conflicting, and impede rational standard setting or control by biological monitoring.

As exposure at the TLV and at higher levels produce almost the same concentrations of urinary MMAc (Figure 4.1), it was previously suggested $[5,9,8]$ that DMAc metabolism might be saturated at its current TLV/TWA (10 ppm). 


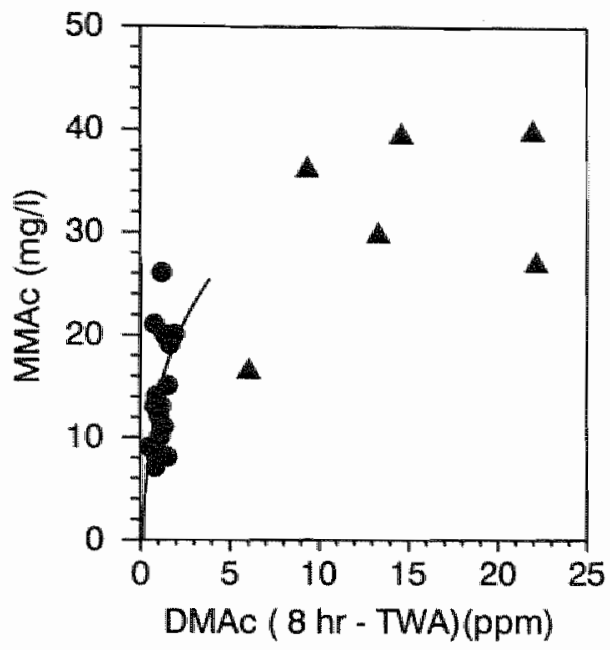

Figure 4.1: Plot of urinary MMAc excretion ( $\left.\mathrm{mg}^{-1}\right)$ versus DMAc exposure (ppm) in workers during 8-hr workshifts. Data were taken from Borm et al. (4) [5] and Kennedy et al. (11]. MMAc concentrations were determined in post-work urimary samples. As urinary volumes were not determined in both studies, we assumed that urine production did not vary to a great extent. The data indicate saturation of MMAc excretion/DMAc metabolism around its current threshold limit value $(10 \mathrm{ppm})$.

Since the liver is both the primary site of DMAc metabolism and the ultimate target for its in wivo toxicity, we set out to study DMAc metabolism and kinetics in isolated perfused rat liver. The ultimate purpose was to evaluate our data with respect to possible saturation of metabolism.

Dimethylformamide (DMF), a homologue of DMAc, is N-demethylated in witro and in vivo by microsomal enzymes [2]. To evaluate a similar conversion for DMAc we tested whether pretreatment of the rats with cimetidine, an inhibitor of cytochrome P-450 dependent N-dealkylation $[4,17]$, or addition of cimetidine to the perfusion medium inhibits DMAc clearance.

\section{Methods}

\section{Chemicalls}

Dimethylacetamide (DMAc), monomethylacetamide (MMAc) dimethylformamide and 4-dimethylaminoantipyrine were obtained from Janssen Chimica (Beerse, Belgium), bovine serum albumin (BSA) from Organon Teknika (Boxtel, 
The Netherlands), lactate dehydrogenase (LDH, from rabbit muscle) EC 1.1.1.27 and glucose 6-phosphate dehydrogenase EC 1.1.1.49 from Boehringer (Mannheim, FRG) and cimetidine, NADP and glucose 6-phosphate from Sigma (St. Louis, U.S.A.). Water was demineralized and filtrated before use. All other chemicals were of analytical grade quality.

\section{Animals}

Male Wistar rats (Bor: WISW (SPFCpb)) weighing 250-280 g were used; about $12 \mathrm{hr}$ before the start of an experiment animals were deprived of food (SRMA 1210, Hope Farms), but had access to water. Cimetidine pretreated rats (75 $\mathrm{mg} \cdot \mathrm{kg}^{-1}$ per day per rat) were injected i.p. with cimetidine in saline during three days prior to the experiment. Phenobarbital was given orally $\left(1{\left.\mathrm{~g} \cdot \mathrm{l}^{-1}\right)}^{-1}\right.$ during one week prior to the preparation of phenobarbital-induced microsomes.

\section{Preparation of microsomes}

Animals were anaesthetized using pentobarbital (Narcovet ${ }^{T M} ; 0.2 \mathrm{ml} \cdot 100 \mathrm{~g}^{-1}$ rat). Livers were removed and homogenized in three volumes of ice-cold phosphate buffer ( $50 \mathrm{mM}$ and $\mathrm{pH} \mathrm{7.4)}$, using glass Potter tubes with a Teflon pestle. The homogenate was centrifuged at $9000 \times \mathrm{g}$ for 20 minutes at $4^{\circ} \mathrm{C}$. The microsomal fraction was sedimented from the supernatant by 40 minutes centrifugation at $110000 \times \mathrm{g}$ and $4^{\circ} \mathrm{C}$. The pellet was resuspended in the same ice-cold buffer and ciluted so that the microsomal fraction of $3 \mathrm{~g}$ liver was diluted in $1 \mathrm{ml}$ buffer.

\section{Microsomal incubations}

The inhibitory effect of cimetidine on cytochrome P-450 activity $[4,17]$, was evaluated by determination of microsomal dimethylaminoantipyrine (DMAP) demethylation activity. The incubations were performed according to Bast [3]. However, the microsomal protein content was $1 \mathrm{mg} \cdot \mathrm{ml}^{-1}$, the semicarbazide concentration was $1 \mathrm{mM}$ and the glucose 6-phosphate dehydrogenase activity was $1 \mathrm{IU} \cdot \mathrm{ml}^{-1}$. Microsomes of animals pretreated with cimetidine and microsomes to which cimetidine was added during the incubation $(1 \mathrm{mM})$ were both tested. When no cimetidine was added to incubations with phenobarbital pretreated microsomes $(n=2)$, formaldehyde concentrations were $127.0 \mu \mathrm{M}$ compared to $25.5 \mu \mathrm{M}$ when cimetidine was added to the incubations $(\mathrm{n}=2)$. Pretreatment of animals with cimetidine had no effect on microsomal DMAP metabolism.

\section{Perfused rat liver: preparation and experiments}

The liver perfusion and all surgical procedures were done according to Meijer et al. [14]. Bicarbonate buffered Krebs-solution with $1 \%$ bovine serum allbu$\min$ (BSA) and $11 \mathrm{mM}$ glucose was filtered $(0.2 \mu \mathrm{m})$ and used as perfusate. 
DMAc was added to the perfusion medium before the start of the perfusion. DMAc concentrations of about $30,50,100$ and $275 \mu \mathrm{M}$ were tested. Medium flow was kept constant during perfusion. Flow was regulated by changing hydrostatic pressure. Bile and perfusate samples were taken every 5 minutes after an initial stabilization period of 10 minutes. Bile secreted during perfusion was expressed as milligram bile per minute per gram liver. Liver damage was assessed through $\mathrm{LDH}$-activity (EC 1.1.1.27) in perfusate samples, using the NADH consumption during pyruvate transformation to lactate [12]. Albumin was added to the perfusate samples (final concentration $3 \%$ ) which were then stored at $-80^{\circ} \mathrm{C}$ until determination. Histological preparations (haematoxilinmeosin staining) of a liver sample were made 1 hour after the initiation of all experiments and examined by light microscopy. The liver was homogenized using a Potter-Elvehjem homogeniser and assayed for DMAc.

\section{Analytical procedures}

Samples of liver perfusate were saturated with $\mathrm{NaCl}$ and extracted with an equal volume of acetonitrile. After addition of an internal standard (DMF; final concentration $36 \mu \mathrm{M}$ ), $0.2 \mu \mathrm{l}$ of the extracted sample was analyzed for DMAc and MMAc by gas chromatography. A Carlo Erba 5300 MS gas chromatograph fitted with a DB-Wax $20 \mathrm{M}$ column (internal diameter $0.326 \mathrm{~mm}$; length, $30 \mathrm{~m}$; film thickness, $0.5 \mu \mathrm{m}$ ) and an on-column injection unit was used. Column temperature was kept at $120^{\circ} \mathrm{C}$, and the detector (flame ionization) temperature was set at $240^{\circ} \mathrm{C}$. Helium was used as a carrier-gas at a flow rate of $3 \mathrm{ml} \cdot \mathrm{min}^{-1}$. Under these conditions the retention times for DMAc and MMAc were 5.2 and 12.2 minutes, respectively. Peak-height ratios of DMAc and dimethylformamide were determined and DMAc concentrations in the perfusate samples were calculated. MMAc concentrations were determined according to the same procedure. Under these conditions the detection limits were 10 and $35 \mu \mathrm{M}$ for DMAC and MMAc, respectively. To evaluate the experimental error, the coefficient of variation (SD $\times 100 \% /$ mean) for DMAc and MMAc measurements were determined. At the detection limit for DMAc $(10 \mu \mathrm{M})$ this was $12 \%$; for concentrations $\geq$ $20 \mu \mathrm{M} 4 \%$. At the detection limit for MMAc $(35 \mu \mathrm{M})$ this was $58 \%$; for concentrations $>35 \mu \mathrm{M} 6 \%$. Final concentrations in the perfisates were corrected for the decrease in medium volume as a result of sampling (see below).

\section{Calculation of extraction ratios}

As samples were taken by redirection of the medium flow from the liver towards the main vessel to a sample vial, the DMAc concentration in the perfusion system was constant during the time that, samples were taken, resulting in an undervaluation of the decline in DMAc medium concentration as a consecuence of DMAc metabolism by the liver. This would result in an underestimation of the 
extraction ratio for DMAc. The incoming DMAc medium concentration $\left(C_{\text {in }}\right)$ was calculated using the samples of the outcoming DMAc medium concentram tion $\left(C_{\text {out }}\right)$, supposing ideal mixture of the outcoming perfusate with the rest of the medium. $C_{\text {out }}$ values between the measurements were interpolated assurning exponential decrease. $C_{\text {in }}$ values were calculated as:

$$
C_{\mathrm{in}}=\frac{A_{\mathrm{in}}}{V} d A_{\mathrm{in}}=\left(Q_{\mathrm{in}} C_{\mathrm{in}}-Q_{\text {out }} C_{\text {out }}\right) d t
$$

As the DMAc concentration does not change during the time samples were taken $\left(Q_{\text {out }}=0\right)$, those periods were not used for further evaluation. The volume of distribution was calculated as the DMAc dose divided by the DMAc medium concentration at the start of the perfusion, obtained by linear extrapolation of a $\log$ linear concentration versus time plot. Extraction ratios $(E)$ were calculated by dividing the difference between $C_{\mathrm{in}}$ and $C_{\text {out }}$, and $C_{\mathrm{in}}$ :

$$
E=\frac{C_{\mathrm{in}}-C_{\mathrm{out}}}{C_{\mathrm{in}}}
$$

Extraction ratios were integrated during a 40 minutes perfusion period starting 10 minutes after the onset of the perfusion and divided by the time period the integral was taken, to level variance in the calculated extraction ratios.

\section{Calculation of airborne concentrations leading to saturation}

DMAc clearance $(\mathrm{Cl})$ was calculated as medium flow times the extraction ratio:

$$
C l=Q \cdot E
$$

DMAc clearance times DMAc medium concentration $\left(c_{1}\right)$ equals metabolic rate $(v):$

$$
v=\mathrm{Cl} \cdot \mathrm{c}_{1}
$$

At steady-state the uptake of airborne DMAc equals its metabolic elimination rate and can be calculated by multiplying alveolar ventilation $\left(\dot{V}_{\text {alv }}\right)$, airborne concentrations of DMAc and DMAc retention ratio $(R)$ :

$$
v=c_{2} \cdot \dot{\mathrm{V}}_{\mathrm{alv}} \cdot R
$$

wherein $\left(c_{2}\right)$ is the concentration of DMAc in air. 


\section{Statistics}

Inlet DMAc medium concentrations of every perfusion were calculated using perfusate DMAc concentrations, which were measured at fixed time intervals. Inlet DMAc medium concentrations were divided in five subgroups, mean extraction ratios and standard deviations were calculated. Differences in extraction ratios were tested using the Kruskal-Wallis and the Wilcoxon two-sample test. The statistical analyses were performed with SAS software.

\section{Results}

\section{General effects}

Table 4.1: Liver weight, bile production and LDH leakage in rat livers perfused at various concentrations of DMAc.

\begin{tabular}{lllll}
\hline $\mathrm{n}$ & $\begin{array}{l}\mathrm{C}_{0} \\
(\mu \mathrm{M})\end{array}$ & $\begin{array}{l}\text { liver weight } \\
(\mathrm{g})\end{array}$ & $\begin{array}{l}\text { bile production } \\
\left(\mathrm{mg} \cdot \mathrm{min}^{-1} \cdot \mathrm{g}^{-1}\right)\end{array}$ & $\begin{array}{l}\mathrm{t}_{L D H} \\
(\mathrm{~min})\end{array}$ \\
\hline 3 & 0.0 & $10.8 \pm 1.8$ & $1.03(0.94,1.11)$ & $40 \pm 5$ \\
2 & $32.6(31.8,33.3)$ & $8.3(7.5,9.0)$ & $0.53(0.38,0.68)$ & $38(20,55)$ \\
3 & $57.5 \pm 5.8$ & $7.6 \pm 0.6$ & $1.12 \pm 0.43$ & $40 \pm 5$ \\
3 & $118.7 \pm 15.2$ & $8.5 \pm 1.4$ & $1.03 \pm 0.20$ & $47 \pm 13$ \\
3 & $273.7 \pm 23.6$ & $7.9 \pm 1.8$ & $0.49(0.32,0.66)$ & $43 \pm 3$ \\
\hline
\end{tabular}

$C_{0}$ is the DMAc medium concentration at the start of the perfusion. $t_{L D F}$ is the time period after the start of the perfusion at which a LDH activity in the perfusate of $100 \mathrm{U}^{-1} \mathrm{I}^{-1}$ was reached.

The mean liver mass, weighed at the end of each perfusion, was $8.6 \mathrm{~g}$ ( $\mathrm{SD}=1.7$; $\mathrm{n}=14$ ). Mean medium flow was $4.2 \mathrm{ml} \cdot \mathrm{min}^{-1} \cdot \mathrm{g}^{-1}(\mathrm{SD}=0.4 ; \mathrm{n}=14)$. When LDH concentrations were plotted against time, LDH leakage remained low during a period of about 40 minutes. Thereafter a steep rise in $\mathrm{LDH}$ leakage occurred. The $100 \mathrm{U} \cdot \mathrm{I}^{-1}$ threshold gives a good measure for the length of the stable period (Table 4.1; see Palmen 1992 [15] for an illustration of this phenomenon). Addition of DMAc to the medium did not influence the results.

Mean bile production was $0.88 \mathrm{mg} \cdot \mathrm{min}^{-1} \cdot \mathrm{g}^{-1}(\mathrm{SD}=0.36 ; \mathrm{n}=12)$ and was not influenced by the addition of DMAc to the medium. Histological preparations of liver samples perfused with DMAc (haematoxillin-eosin staining), were normal in appearance (Figure 4.2). 


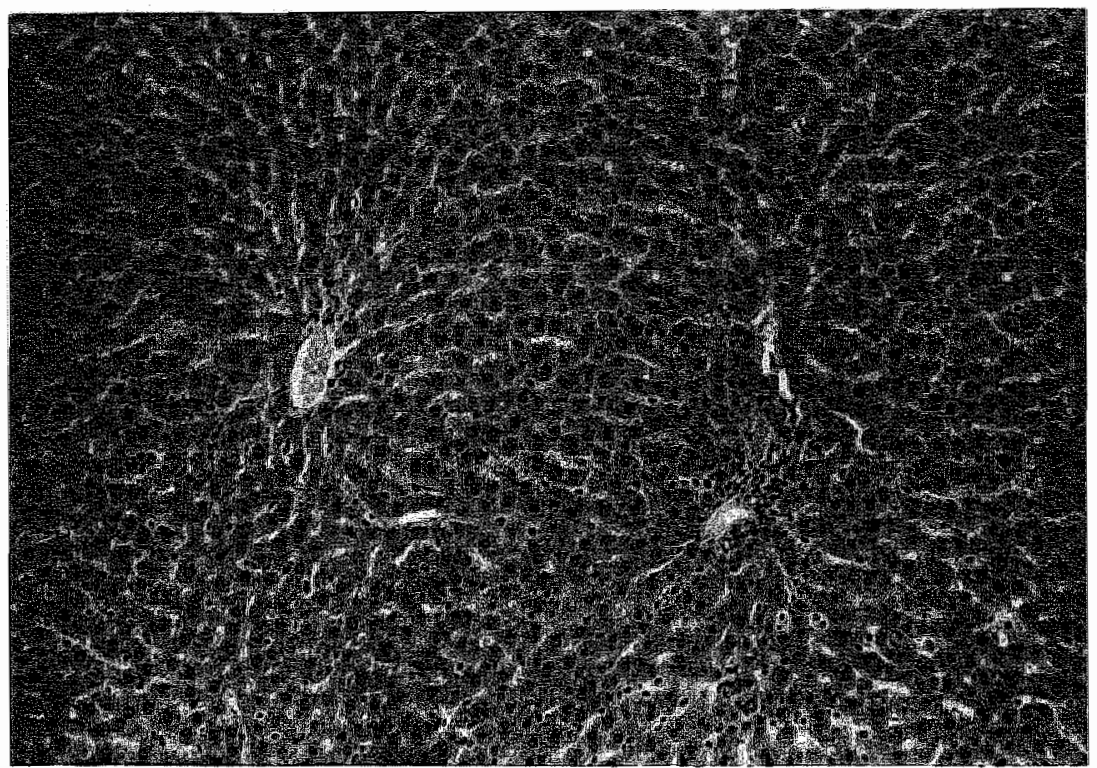

Figure 4.2: Hüstological evaluation of a liver perfused during a one hour recircallating perfusion after haematoxilin-eosin staining. Medium was composed of a bicarbonate buffered Krebs solution with $1 \%$ albumin and glucose. $100 \mu \mathrm{M}$ DMAc was added to the medium.

Rats pretreated with cinnetidine or addition of cimetidine during the perfusion

The mean liver mass of livers pretreated with cimetidine was $9.7 \mathrm{~g}(8.3,11.1$; $\mathrm{n}=2)$ and $6.6 \mathrm{~g}(6.2,6.9 ; n=2)$ when cimetidine was added during the perfusion. Mean medium flow was $3.8 \mathrm{mll} \cdot \mathrm{min}^{-1} \cdot \mathrm{g}^{-1}(3.2,4.3 ; n=2)$ when rats were pretreated with cimetidine and $4.2 \mathrm{ml} \cdot \mathrm{min}^{-1} \cdot \mathrm{g}^{-1}(4.1,4.2 ; \mathrm{n}=2)$ when cimetidine was added to the medium (Table 4.2). The time period after the start of the perfusion at which a LDH activity of $100 \mathrm{U} \cdot \mathbb{1}^{-1}$ was reached was 60 minutes or more when rats were pretreated with cimetidine and 30 minutes when cimetidine was added to the medium compared with 47 minutes when no cimetidine was used (Table 4.2). These differences were not statistically significant. Mean bile production was $0.91 \mathrm{mg} \cdot \mathrm{min}^{-1} \cdot \mathrm{g}^{-1}$ liver $(0.82,0.99 ; \mathrm{n}=2)$ when rats were pretreated with cimetidine and $0.81 \mathrm{mg} \cdot \min ^{-1} \cdot \mathrm{g}^{-1}$ liver $(0.64,0.97 ; \mathrm{n}=2)$ when cimetidine was added to the medium, and was also not different within two standard deviations from perfusions without cimetidine. 
Table 4:2: Effect of cimetidine on rat liwer function during perfusion with DMAc.

\begin{tabular}{llllll}
$\begin{array}{l}\text { perfusion } \\
\text { type }\end{array}$ & $\mathrm{n}$ & $\begin{array}{l}\mathrm{C}_{0} \\
(\mu \mathrm{M})\end{array}$ & $\begin{array}{l}\text { liver mass } \\
(\mathrm{g})\end{array}$ & $\begin{array}{l}\text { bile production } \\
\mathrm{mg} \cdot \mathrm{min}^{-1} \cdot \mathrm{g}^{-1}\end{array}$ & $\begin{array}{l}\mathrm{t}_{\text {LDM }} \\
(\mathrm{min})\end{array}$ \\
\hline $\mathrm{A}$ & 3 & $118.7 \pm 15.2$ & $8.5 \pm 1.4$ & $1.03 \pm 0.20$ & $47 \pm 13$ \\
$\mathrm{~B}$ & 2 & 101.9 & 9.7 & 0.91 & $>60$ \\
$\mathrm{C}$ & & $(95.2,108.6)$ & $(8.3,11.1)$ & $(0.82,0.99)$ & $(>60,60)$ \\
& 2 & 91.4 & 6.6 & 0.81 & 30 \\
& & $(89.5,93.4)$ & $(6.2,6.9)$ & $(0.64,0.97)$ & $(30,30)$ \\
\hline
\end{tabular}

Perfusion type: (A) no additions (B) cimetidine pretreatment (C) cimetidine added to the medium. $\mathrm{C}_{0}$ (values are mean $\pm \mathrm{SD}$ ) is the DMAc medium concentration at the start of the perfusion. $t_{L D H}$ is the time period after the start of the perfusion at which a LDH activity in the perfusate of $100 \mathrm{U} \cdot 1^{-1}$ was reached.

\section{Determination of DMAc extraction ratios}

Tissue DMAc concentrations were determined immediately after the cessation of the experiments and found to be negligible. The estimated volume of distribution for DMAc was $165 \mathrm{ml}$, indicating almost no protein binding, as $150 \mathrm{ml}$ medium was used in the perfusion. DMAc inlet concentrations were calculated using perfusate DMAc concentrations and subsequently divided in five subgroups. Mean DMAc perfusate concentrations of these subgroups were $16(n=29), 36(n=50)$, $70(n=34), 160(n=18)$ and $225(n=18) \mu M$. Mean extraction ratios for the respective concentrations were $0.085,0.061,0.066,0.047$ and 0.044 . DMAc extraction ratios were different at varying perfusate concentrations (Kruskal-Wallis; $p=0.074$ ) (Figure 4.3).

The extraction ratio at a medium concentration of $16 \mu \mathrm{M}$ was significantly higher than the extraction ratio at DMAc inlet concentrations of $36 \mu \mathrm{M}, 70 \mu \mathrm{M}, 160 \mu \mathrm{M}$ or $225 \mu \mathrm{M}$ (Figure 4.3). Extraction ratios did not differ significantly between groups with DMAc perfusate concentrations of $36 \mu \mathrm{M}$ or more.

As cimetidine is reported to inhibit both the monooxygenase and oxidase activity of cytochrome P-450 $[4,18]$ the effect of cimetidine pretreatment or addition of cimetidine to the perfusion medium on DMAc extraction ratios was tested. As cimetidine perfusions were performed with starting DMAc concentrations of $100 \mu \mathrm{M}$, control perfusions with the same initial DMAc concentrations were selected. Inlet concentrations between 25 and $100 \mu \mathrm{M}$ DMAc were compared between the groups (Figure 4.4).

Based on the results we may conclude that neither pretreatment of the rats with 


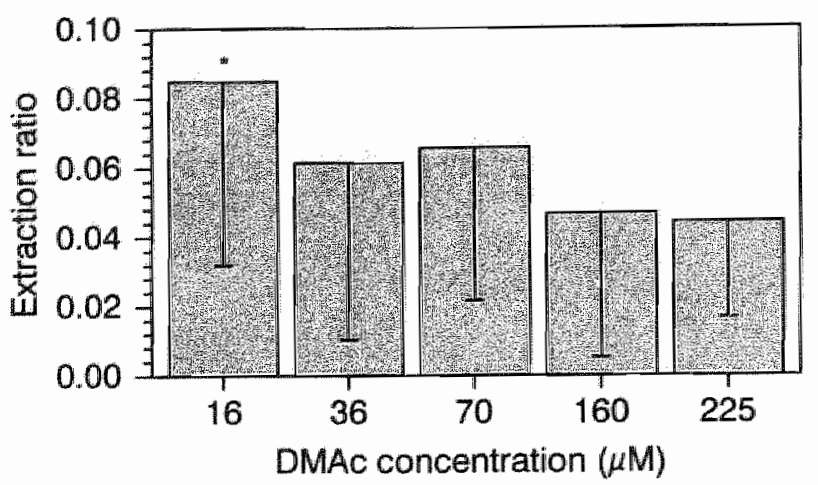

Figure 4.3: Mean dimethylacetamide (DMAc) extraction ratios plus standard deviations at varying DMAc inlet concentrations. The extraction ratio at a DMAC inlet, concentration of $16 \mu \mathrm{M}$ was significantly highex (Wilcoxon) compared to DMAc concentrations of $36 \mu \mathrm{M}(p=0.039), 70 \mu \mathrm{M}(p=0.079), 160 \mu \mathrm{M}(p=$ $0.007)$ and $225 \mu \mathrm{M}(p=0.010)$, indicated by $(*)$.

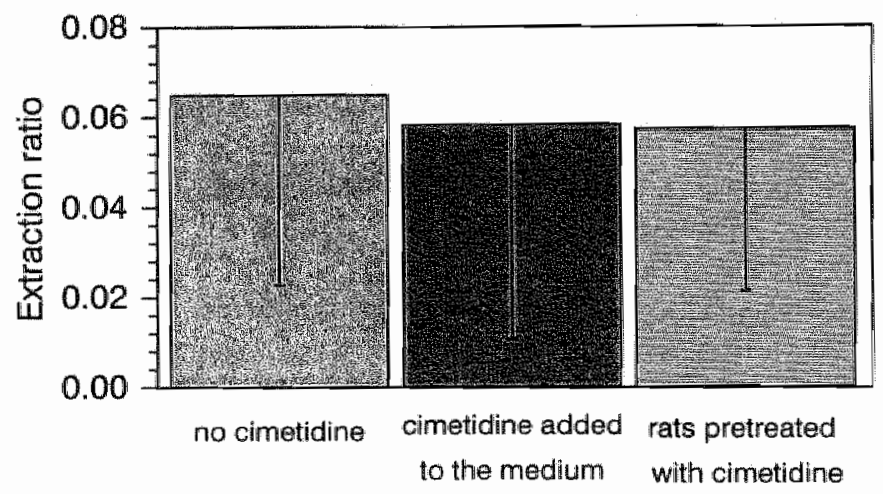

Figure 4.4: No effect of cimetidine addition to the perfusion medium or pretrentment of the rats with cimetidine on dimethylacetamide extraction ratio (SD). Inlet DMAc concentrations were between 25 and $100 \mu \mathrm{M}$.

cimetidine $\left(75 \mathrm{mg} \cdot \mathrm{kg}^{-1}\right.$ rat) during three days before perfusion, nor addition of cimetidine to the perfusion medium 20 minutes after the start of the perfusion resulted in a decreased extraction of DMAc.

\section{Calculation of airborne concentrations leading to saturation}

The starting point for this calculation is the lowest perfusion concentration at which saturation of rat liver extraction was observed (36 $\mu \mathrm{M}$; Figure 4.3). We 
assumed that in humans saturation occurs at the same (plasma) level and used (extrapolated) rat data to calculate the airborne exposure at which such a plasma level is achieved.

Kinetic parameters in rat livers at the concentration where saturation occurs $(36 \mu \mathrm{M})$ :

\begin{tabular}{ll}
\hline mean rat liver weight & $8.6 \mathrm{~g}$ \\
mean medium flow & $4.2 \mathrm{ml} \cdot \mathrm{min}^{-1} \cdot \mathrm{g}^{-1}$ \\
Extraction ratio & 0.061 \\
Clearance & $2.20 \mathrm{ml} \cdot \mathrm{min}^{-1}$
\end{tabular}

Extrapolation of these findings to a human liver $(1.5 \mathrm{~kg}$ ) leads to a DMAc clearance of $380 \mathrm{ml} \cdot \mathrm{min}^{-1}$ and a maximal metabolic rate of $14 \mu$ moles $\mathrm{min}^{-1}$. The respiratory rate at modest physical exercise $(50 \mathrm{~W})$ is about $20 \mathrm{l} \cdot \mathrm{min}^{-1}[1]$. Assuming a retention ratio of $100 \%$ an uptake equal to this metabolic rate would be reached at an airborne DMAc concentration of $18 \mathrm{ppm}$.

\section{Discussion}

To prevent its hepatotoxic activity at chronic exposure [5], the TLV for DMAc was set at $1.0 \mathrm{ppm}(8 \mathrm{hr}$, TWA). As the concentration of MMAc, a metabolite of DMAc, in urine might be a good parameter for biological monitorimg of DMAc exposure, a deeper understanding of kinetics and metabolism of DMAc is desirable. Particularly, when metabolism of DMAc is saturated, no further increase in urine MMAc concentrations at higher exposures can be expected. As previous data (Figure 4.1) suggest that DMAc metabolism might be saturated at its current TLV/TWA $\left(10 \mathrm{ppm}=35 \mathrm{mg} \cdot \mathrm{m}^{-3}\right)$, we used the isolated perfused rat. liver to gain an insight into DMAc kinetics and metabolism.

Our data show that the extraction ratio at a DMAc medium concentration of $16 \mu \mathrm{M}(\mathrm{E}=0.085 ; \mathrm{SD}=0.053 ; \mathrm{n}=29)$ was significantly higher than the extraction ratio at $36 \mu \mathrm{M} \mathrm{DMAc}(\mathrm{E}=0.061 ; \mathrm{SD}=0.051 ; \mathrm{n}=50)$. The extraction ratios at DMAc medium concentrations of $36 \mu \mathrm{M}$ or higher were not different from each other. Based on these findings we have concluded that DMAc metabolism was saturated at a DMAc medium concentration of $36 \mu \mathrm{M}$. As the detection limit of DMAc was $10 \mu \mathrm{M}$, it was not possible to test DMAc medium concentrations lower than $10 \mu \mathrm{M}$. For this reason, a DMAc medium concentration group below $16 \mu \mathrm{M}$ could not be tested. Therefore, it can not be excluded that saturation already occurs at even lower concentrations. 
Bile production and LDH leakage into the medium were not infuenced by the addition of DMAc to the medium; histological examination of liver preparations perfused with DMAc (initial inlet concentration $100 \mu \mathrm{M}$ ) were normal, so liver impairment is not likely to be the cause of the lower extraction ratio.

As the analogue of DMAc, DMF, is N-demethylated by microsomal enzymes [2] and as both DMF and DMAc are demethylated, the two substances could possibly share the same metabolic pathway. Cimetidine, reported to inhibit N-demethylase activity of cytochrome $P-450[4,17]$, was tested with respect to its effect on DMAc extraction ratios. Neither cimetidine pretreatment nor addition of cimetidine to the medium had an effect on bile production or $\mathrm{LDH}$ activity. Also pretreatment of animals with cimetidine ( $75 \mathrm{mg} \cdot \mathrm{kg}^{-1}$ per day) during the three days before the experiment, had no effect on microsomal DMAP metabolism or DMAc extraction. ratio (Figure 4.4). The absence of an effect on DMAP N-demethylase activity indicates a recovery of this activity in microsomes after the final injection, given 10 hours before the preparation of the microsomes. Speeg et al. previously reported a full recovery of DMAP activity after 24 hours, but they did not check DMAP activity after shorter periods of time [18]. The absence of an effect of cimetidine addition on DMAc clearance in the perfused liver indicates that DMAc may be unetabolised by other (iso)enzymes than DMAP.

Experimental error was low as the coefficient of variation of the analytical procedure for DMAc was $4 \%$ for all DMAc concentrations above $10 \mu \mathrm{M}$. The large variation in the results shown in Figures 4.3 and 4.4 was caused by large interindividual metabolic variation of the Wistar rats. As Wistar is an outbred strain, large inter-individual variation was to be expected. This variation in DMAc metabolism will probably lead to variation in the DMAc concentration at which saturation occurs. This knowledge might be of major concern regarding possible human variation in DMAc saturation. Variation in DMAc metabolism in humans was already suggested by Borm et al, who found in two out of eight workers a significantly higher MMAc/DMAc ratio in urine [5].

The airborne DMAc concentration that would lead to a saturated metabolism in humans was based on the medium DMAc concentration at which saturation occurred in rat livers. Assuming modest physical exercise $(50 \mathrm{~W})$ with a respiratory ventilation of $201 \cdot \mathrm{min}^{-1}$, it was calculated that saturation would occur at an air-borne DMAc concentration of $18 \mathrm{ppm}$. This implies that interpretation of data obtained for biological monitoring at values exceeding $25 \mathrm{mg}^{-1}{ }^{-1} \mathrm{MMAc}$ in urine, should proceed with utmost care (Figure 4.1).

Acknowledgements The authors want to thank Alois Sesink and Toon van Bommel for their technical support, Henny Roelofs, Department of Gastroenterology (University of Nijmegen) and Dr GMM Groothuis, Department of Pharmacology and Therapeutics (University of Groningen), for their advice in performing liver perfusions and the De- 
partment of Pathology from the Academic Hospital Maastricht for the preparation of histological coupes. 


\section{Bibliography}

[1] Astrand, I. Effect of physical exercise on uptake, distribution and elimination of vapors in man, in: Fiserova, V. (ed.), Modelling of inhalation exposure to vapors: uptake, distribution, and elimination, Vol II, CRC Press, Boca Raton, USA, 1983.

[2] Barnes, J.R. and Ranta, K.E. (1972) The metabolism of Dimethylformamide and Dimethylacetamide. Toxicol. Appl. Parmacol. 23, 271-276.

[3] Bast, A. and Noordhoek, J. (1981) Evaluation and comparison of colorimetric, radiometric and high performance liquid chromatography assays for aminopyrineN-demethylation by rat liver microsomes. J. Pharm. Pharmacol. 33, 14-18.

[4] Bast, A., Smid, K. and Timmerman, H. (1989) The effects of cimetidine, ranitidine and famotidine on rat hepatic microsomal cytochrome P-450 activities. Agents and actions 27, 188-191.

[5] Borm, P.J.A., DeJong, L. and Vliegen, A. (1987) Environmental and biological monitoring workers occupationally exposed to dimethylacetamide. J. of Occup. Med. 29, 898-903.

[6] Caujolle, F., Chanh, P.H., Dat-Xuong, N. and Azum-Gelade, M.C. (1979) Toxicological studies upon acetamide and its $\mathbb{N}$-methyl and $\mathrm{N}$-ethyl derivatives. Arzneim. Forsch. 20, 1242-1246.

[7] Corsi, G.C. (1971) Dimethylacetamide-induced occupational disease with particular tention to hepatic functions. Med. Lav. 62, 28-42.

[8] Fiserova-Bergerova, V. (1990) Application of toxicokinetic models to estimate biological exposure indicators. Ann. Occup. Hyg. 34, 639-651.

[9] Fiserova-Bergerbva, V. The use of models to establish biological exposure indicators, in: International workshop on pharmacokinetic modelling in occupational health, Switserland, Leysin, 1990.

[10] Horn, H.J. (1961) Toxicology of dimethylacetamide. Toxicol. Appl. Pharmacol. 3, $12-24$.

[11] Kennedy, G.L and Pruett, J.W. (1989) Biologic monitoring for dimethylacetamide: measurement for 4 consecutive weeks in a workplace. J. Occup. Med. 31, 47-50.

[12] Kreutzer, H.J.H. Klinisch-chemisch laboratorium onderzoek, Elsevier, Amsterdam, 1982 .

[13] Maxfield, M.E., Barnes, J.R., Azar, A. and Trochimowicz, H.T. (1975) Urinary excretion of metabolite following human exposure to dimethylformamide or dimethylacetamide. J. Occup. Med. 17, 506-511.

[14] Meijer, D.K.F., Keulemans, K. and Mulder, G.J. (1981) Isolated perfused rat liver technique. Methods Enzymol. 77, 81-94.

[15] Palmen, N.G.M., Evelo, C.T.A. and Henderson, P.Th. (1992) Influence of oxygen supply on liver condition and elimination of dimethylacetamide in the isolated perfused rat liver. Toxicol. in Vitro 6, 357-365.

[16] Sherman, H. and Barnes, J.A. as referenced by Kennedy, G.L. (1986) Biological effects of acetamide, formamide and their monomethyl and dimethyl derivatives. CRC Crit. Rev. Toxicol. 17, 129-182.

[17] Somogyi, A. and Muirhead, M. (1987) Pharmacokinetic interactions of cimetidine. Clin. Pharmacokin. 12, 321-366. 
[18] Speeg jr., K.V., Patwardhan, R.V., Avant, G.R., Mitchell, M.C. and Sehenker, $\mathrm{S}$. (1982) Inhibition of microsomal drug metabolism by histamine $\mathrm{H}_{2}$-receptor antagonists studied in vivo and in vitro in rodents. Gastroenterology 82, 89-96. 



\section{Chapter 5}

\section{Influence of oxygen supply on liver condition and elimination of dimethylacetamide in the isolated perfused rat liver}

Palmen $^{1}$, N.G.M., Evelo ${ }^{1}$, C.T.A., Borm ${ }^{2}$, P.J.A. and Henderson ${ }^{3}$, P.Th.

1 Department of Pharmacology, Section Toxicology

2 Department of Health Risk Analysis

University of Limburg, PO Box 616, 6200 MD Mastricht

3 TNO Medical Biological Laboratory, PO Box 5815, 2280 HV Rijswijk, The Netherlands.

Published in: Toxicology in Vitro 6 (1992) 357-365. 


\section{Summary}

The effects were studied of improved oxygen supply on the integrity and metabolic activity towards dimethylacetamide of the isolated perfused rat liver. Improvement of oxygen supply by increased medium oxygenathon or addition of chemical oxygen carriers (perfuortributylamine) or erythrocytes led to increased bile secretion. Leakage of lactate dehydrogenase and aspartate aminotransferase could be prevented during a one hour perfusion when either chemical oxygen carriers or erythrocytes were added. Improwed medium oxygenation alone was not sufficient to prevent high enzyme leakage during the second half of the perfusion period. Histological evaluation confirmed the conclusion that less damage occurred when erythrocytes or perfluortributylamine were added to the perfusion medium. The metabolic clearance of dimethylacetamide by the perfused rat liver was not significantly improved when erythrocytes were added to the medium. The results show that addition of perfluortributylamine, or erythrocytes at a level of $4 \mathrm{~g}$ haemogiobin. $\mathrm{I}^{-1}$, is nescessary to maintain liver integrity for at least 1 bour in the liver perfusion system used in this study.

\section{Introduction}

Biotransformation and toxicity of xenobiotics can be studied by using test systems in vitro. The liver is the organ with the highest metabolic activity [14], and many test systems in vitro are based on this organ. In order of increasing conformity with the situation in wivo these test systems comprise preparations of (1) purified enzymes, (2) microsomes, (3) liver homogenates, (4) isolated hepatocytes, (5) liver slices and finally (6) the (isolated) perfused liver. Advantages of the perfused liver over the more basal systems are the intact cellular structure (which is also found in hepatocytes and liver slices) and the presence of all cell types in their nomal cellular architecture. Medium flow can be kept constant and bile samples can be taken. Compared to the situation in vivo, liver metabolism can be studied without interference from metabolic processes in other organs; Substances in concentrations that are not tolerated in vivo can be thested.

A standard liver perfusion technique does not exist. Variation between experimenters exists with regard to animal species, liver perfusion systems, composition of the medium, medium pressure and medium flow through the liver. For this reason it is difficult to compare liver condition between studies. In contrast to the conviction of many authors that medium without oxygen carriers keeps the liver intact for several hours, in a well-documented study Schmucker and Curtis had already reported in 1974 that this was not the case [18], even when the How of medium through the liver was increased. However, when erythrocytes were added 
to the perfusion medium, liver medium flow rates of $15 \mathrm{ml} \cdot \mathrm{min}^{-1} \cdot \mathrm{g}^{-1}$ liver were sufficient to maintain liver integrity. This is in agreement with the findings of Sies [19] who reported that oxygen saturation of the medium is a critical parameter during perfusion of the isolated liver. Early cell impairment may be a consequence of oxygen deficiency, which may affect kinetic and metabolic pardmeters. However, oxygen can also be toxic when its concentration in the medium is too high [5]. For use in the technique using the isolated perfused rat liver in the study of the metabolism of xenobiotics [12], we have studied the effects of various methods of increasing the oxygen supply to the liver. For this purpose, the addition of chemical oxygen carriers or erythrocytes to the medium, and changes in flow of the oxygenating medium were used. To our knowledge the effect of these medium conditions on liver integrity has not previously been tested in the same liver perfusion system in a single study. As liver medium flow rates of more than $3.5 \mathrm{ml} \cdot \mathrm{min}^{-1} . \mathrm{g}^{-1}$ liver were damaging in their own right, we did not increase oxygen supply by increasing the flow rate of medium to the liver itself. Finally, kinetic parameters of dimethylacetamide were determined using both medium without addition of oxygen carriers and medium supplied with erythrocytes.

\section{Materials and methods}

\section{The isolated perfused rat liver}

\section{Chemicals}

Dimethylacetamide (DMAc), dimethylformamide and monomethylacetamide were supplied by Janssen Chimica (Beerse, Belgium), bovine serum albumin (BSA) by Organon Teknika (Boxtel, The Netherlands), Synperonic F68 by Serva (Heidelberg, Germany) and perfluortributylamine (FC43) by 3M Company (Leiden, The Netherlands). Lactate dehydrogenase ( $\mathrm{LDH}$; from rabbit muscle) $\mathrm{EC}$ 1.1.1.27 and malate dehydrogenase (from pig heart) EC 1.1.1.37 were obtained from Boehringer (Mannheim, Germany). Water was demineralized and filtered before use. All other chemicals were of analytical grade quality.

\section{Animals}

Male Wistar rats (Bor: WISW (SPFCpb)) weighing 250-280 g were used; they were deprived of food (SRMA 1210, Hope Farms) 12 hours before the start of the experiment, but had free access to water $(\mathrm{pH} 2.5)$. The rats were housed at $21^{\circ} \mathrm{C}$, at a relative humidity of $55 \%$, and were kept on a night /day cycle from 07.00 until 19.00 hour. 


\section{Erythrocytes}

Blood derived from human volunteers was obtained from the Red Cross Blood Bank Zuid Limburg, collected in vacuum vessels in ethylenediaminetetraacetic acid and stored overnight at $4^{\circ} \mathrm{C}$.

\section{Liver perfusion technique}

The liver perfusion and all surgical procedures were done according to Meijer et al. [11]. Medium flow was kept constant during perfusion and regulated by changing hydrostatic pressure. Bile and perfusate samples were taken at regular time intervals. At the end of the perfusion, 1 hour after the start of a perfusion, a liver sample was taken for histological examination.

\section{Perfusion medium}

Standard medium was composed of a bicarbonate buffered Krebs solution with $1 \%$ BSA and $11 \mathrm{mM}$ glucose and was filtered $(0.2 \mu \mathrm{m})$. For erythrocyte-amended medium, the erythrocytes were washed three times in $0.9 \% \mathrm{NaCl}$ and diluted in standard medium to a haemoglobin concentration of $4 \mathrm{~g} \cdot \mathrm{I}^{-1}$. In metabolic experiments, DMAc was added to the medium before the addition of erythrocytes. Medium amended with perfluortributylamine was prepared diluting $5.6 \mathrm{~g}$ of the detergent Synperonic F68 in deionized water $(150 \mathrm{ml})$. After addition of $24 \mathrm{ml}$ perfluortributylamine ( $F C 43$ ), the mixture was sonicated for 1 hour $(40-50 \mathrm{~W}$, $0-4^{\circ} \mathrm{C}$ ) under carbon dioxide. $160 \mathrm{ml}$ of this suspension was added to Krebs buffer (20 times concentrated), glucose and BSA to obtain the same component concentration as in standard medium. The perfuortributylamine medium was filtered $(8 \mu \mathrm{m})$ before use.

\section{Study design}

Liver integrity using different medium conditions was tested during a one hour perfusion period. Oxygen supply to the liver was modified by manipulation of the medium flow rate through the oxygenators by way of an extra loop in medium flow, or by addition of a chemical oxygen carrier (perfluortributylamine) or erythrocytes to the medium. Liver medium flow was $3.5 \mathrm{ml} \cdot \mathrm{min}^{-1} \cdot \mathrm{g}^{-1}$ liver for every perfusion performed. The effect of a high flow rate of medium through the artificial lungs (186 $\mathrm{ml} \cdot \mathrm{min}^{-1} ; \mathrm{n}=3$ ) compared with a low flow rate of medium $\left(92 \mathrm{ml} \cdot \mathrm{min}^{-1} ; n=5\right)$ in standard medium was determined. Perfluortributylaminesupplemented medium $(n=2)$ in perfusions with a low flow rate of medium, and erythrocytes added to perfusions. with a high flow rate of medium $(n=4)$ were also evaluated. Bile production, LDH and aspartate aminotransferase (ASAT; EC 2.6.1.1) activity in perfusate samples were determined and histological preparations were made. 
The biotransformation capacity of the liver was studied by addition of DMAc (90 and $120 \mu \mathrm{M}$ ) to a perfusion medium with and without erythrocytes. A plot of logarithmic concentration $v$. time was used to determine the elimination rate constants $(k)$. Corrections were made for the removal of medium during sampling. Clearance was calculated by multiplying $k$ by the volume of distribution. The extraction ratio was calculated by division of clearance by the actual medium flow.

\section{Analytical procedures}

Bile samples were taken at regular intervals. Bile secreted during perfusion was expressed as mass per minute per gram liver. LDH activity of the perfusate samples was determined according to Kreutzer (1989), ASAT activity according to Moss (1986). Albumin was added to the perfusate samples (final concentration $3 \%$ ) and stored at $-80^{\circ} \mathrm{C}$ until determination. Histological preparations (haematoxilin-eosin staining) of the liver were made immediately after cessation of the experiment and examined by light microscopy.

\section{Gas chromatochraphic analysis}

Samples ( $1 \mathrm{ml})$ were saturated with sodium chloride and extracted with an equal volume of acetonitrile. After addition of an internal standard (dimethylformamide, final concentration $36 \mu \mathrm{M}$ ), $0.2 \mu$ lof the extracted sample was analyzed for DMAc and its metabolite monomethylacetamide by gas chromatography. A Carlo Erba 5300MS (Interscience) gas chromatograph fitted with a DB-WAX $20 \mathrm{M}$ column $(0.326 \mathrm{~mm} \times 30 \mathrm{~m}$, film thickness $0.5 \mu \mathrm{m})$ and an on-column injection unit was used. Column temperature was $120^{\circ} \mathrm{C}$ (isothermal), and the detector (flame ionization detector) temperature was set at $240^{\circ} \mathrm{C}$. Helium was used as a carrier gas at a flow rate of $3 \mathrm{ml} \cdot \mathrm{min}^{-1}$. Under these conditions the retention times for DMAc and monomethylacetamide were 5.2 and 12.2 minutes, respectively. Peak-height ratios of DMAc and dimethylformamide were determined and DMAC concentrations in the perfusate samples were calculated. Under these conditions the detection limits were 10 and $35 \mu \mathrm{M}$ for DMAc and monomethylacetamide respectively. Final concentrations in the perfusate were corrected for the decrease in medium volume. 


\section{Results}

Viability of the isolated perfused rat liver

Bile secretion

Table 5.1: Mean bile secretion of individual perfusions

\begin{tabular}{|c|c|c|}
\hline $\begin{array}{l}\text { Additions to } \\
\text { the standard } \\
\text { medium }\end{array}$ & $\begin{array}{c}\text { oxygenator medium } \\
\text { flow rate } \\
\left(\mathrm{ml} \cdot \mathrm{min}^{-1}\right)\end{array}$ & $\begin{array}{l}\text { mean bile secretion } \pm S D \\
\text { for individual perfusions } \\
\left(\mathrm{mg} \cdot \mathrm{min}^{-1} \cdot \mathrm{g}^{-1} \text { liver) }\right.\end{array}$ \\
\hline \multirow[t]{5}{*}{ None } & 92 & $0.59 \pm 0.28$ \\
\hline & & $0.26 \pm 0.21$ \\
\hline & & $0.77 \pm 0.37$ \\
\hline & & $0.28 \pm 0.11$ \\
\hline & & $0.44 \pm 0.23$ \\
\hline \multirow[t]{3}{*}{ None } & 186 & $0.18 \pm 0.03$ \\
\hline & & $0.97 \pm 0.23$ \\
\hline & & $0.94 \pm 0.09$ \\
\hline \multirow[t]{2}{*}{$\mathrm{FC} 43$} & 92 & $0.80 \pm 0.48$ \\
\hline & & $0.58 \pm 0.30$ \\
\hline \multirow[t]{4}{*}{ Erythrocytes } & 186 & $1.33 \pm 0.17$ \\
\hline & & $1.39 \pm 0.29$ \\
\hline & & $1.16 \pm 0.16$ \\
\hline & & $0.56 \pm 0.06$ \\
\hline
\end{tabular}

Bile secretion of individual perfusions with low and high flow rates over the oxygenators and with and without addition of perfluortributylamine ( $\mathrm{FC43}$ ) or erythrocytes. Meantstandard deviations within the individual perfusions are given.

Bile secretion varied significantly between indiviclual perfusions, even under the same conditions (Table 5.1). The influence of medium conditions on bile secretion is illustrated in Figure 5.1. Data from individual perfusions were standardized with respect to the mean value for the respective medium condition 45 minutes after the start of the perfusion. In all cases, bile production was low directly after the onset of the perfusion and increased to a steady level, which was attained after about 20 minutes. Bile secretion under low oxygen conditions was relatively low $\left( \pm 0.4 \mathrm{mg} \cdot \mathrm{min}^{-1} \cdot \mathrm{g}^{-1}\right.$ liver). Both an increase in oxygen saturation of medium and the addition of standard chemical oxygen cariers resulted in increased bile production ( $\pm 0.6 \mathrm{mg} \cdot \mathrm{min}^{-1} \cdot \mathrm{g}^{-1}$ liver). When erythrocytes were added to the medium at a high flow rate through the oxygenators, the bile secretion was even 


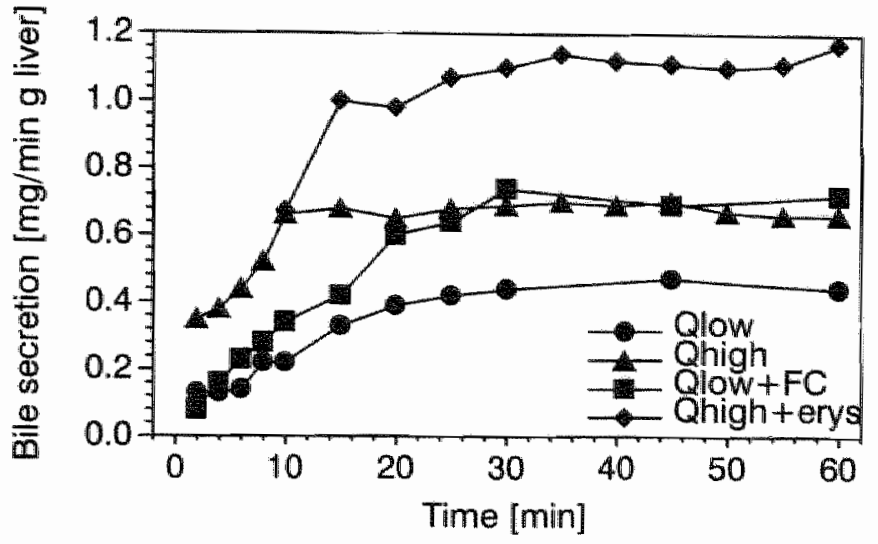

Figure 5. I: Time course of bile secretion during perfusions with standard medium and an oxygenator medium fow of $92 \mathrm{ml} \cdot \mathrm{min}^{-1}\left(\mathrm{Q}_{\text {low }} ; \mathrm{n}=5\right)$; the same medium composition but an oxygenator medium flow of $186 \mathrm{ml} \cdot \mathrm{min}^{-1}\left(\mathrm{Q}_{\text {high }} ; \mathrm{n}=3\right)$; per fluortributylamine (FC43) added to the standard medium with an oxygenator medium flow of $92 \mathrm{ml} \cdot \mathrm{min}^{-1}\left(\mathrm{Qlow}_{\text {low }}+\mathrm{FC} ; \mathrm{n}=2\right)$; ery throcytes $\left(\mathrm{Hb}=4 \mathrm{~g} \cdot \mathrm{l}^{-1}\right)$ added to the standard medium and an oxygenator medium How of $186 \mathrm{ml} \cdot \mathrm{min}^{-1}\left(Q_{\text {high }}\right.$ + erys; $n=4$ ). The values are means of all perfusions performed in that category.

higher ( $\pm 1.1 \mathrm{mg}^{*} \min ^{-1} \mathrm{~g}^{\infty-1}$ liver $)$

\section{Lactate dehydrogenase and aspartate aminotransferase release}

Enzyme leakage during the first 30 minutes after the start of the perfusion was low in all cases. Thereafter, the leakage of LDH and ASAT increased progressively in the absence of oxygen carriers (Figure 5.2). Increased flow rate of the medium through the artificial lungs did not influence enzyme leakage. When perfluortributylamine or erythrocytes were added to the medium, the enzyme leakage remained low during the whole perfusion period. A LDH perfusate activity of $300 \mathrm{U} \cdot \mathrm{I}^{-1}$ and an ASAT activity of $75 \mathrm{U} \cdot \mathrm{I}^{-1}$ were reached in the perfusate after about 45 minutes, when no oxygen carriers were added to the medium; both activities increased markedly thereafter. When chemical oxygen carriers or erythrocytes were addled to the medium, these enzyme activities were not reached within a one hour perfusion period.

\section{Histological evaluation}

Histological evaluation was in agreement with data obtained from enzyme leakage determination. Vacuolization and necrosis in the central lobular area (Rappaport areas 2 and 3) occurred when no chemical oxygen carriers or erythrocytes were 

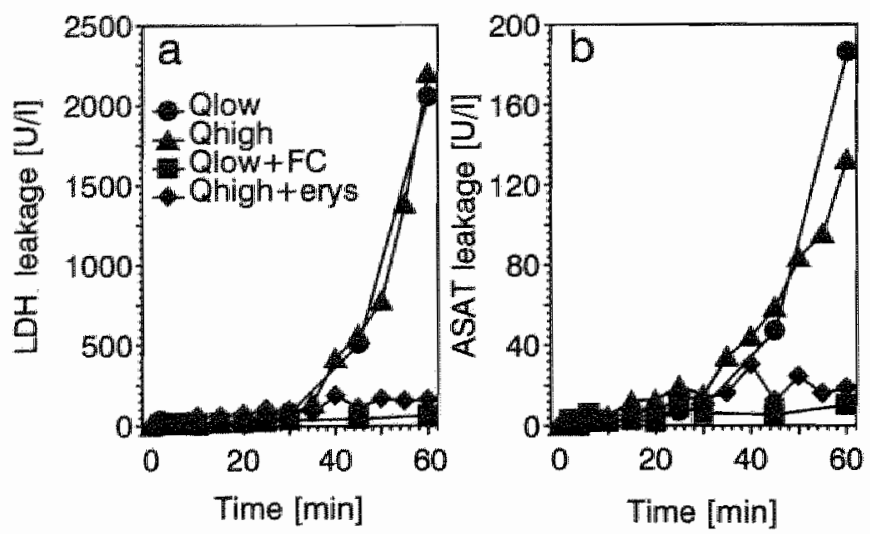

Figure 5.2: Time course of (a) lactate dehydrogenase (LDH) and (b) aspartate aminotransferase (ASAT) activity during perfusions with standard medium and an oxygenator medium flow of $92 \mathrm{ml} \cdot \mathrm{min}^{-1}\left(Q_{\text {low }} ; \mathrm{n}=5\right.$ ); the same medium composition but an oxygenator medium flow of $186 \mathrm{ml} \cdot \mathrm{min}^{-1}\left(Q_{h i g h} ; \mathrm{n}=3\right)$; perfluortributylamine (FC43) added to the standard medium with an oxygenator medium flow of $92 \mathrm{ml} \cdot \mathrm{min}^{-1}\left(Q_{\text {low }}+\mathrm{FC}_{;} \mathrm{n}=2\right)$; erythrocytes $\left(\mathrm{Hb}=4 \mathrm{~g} \cdot \mathrm{l}^{-1}\right)$ added to the standard medium and an oxygenator medium flow of $186 \mathrm{ml} \cdot \mathrm{min}^{-1}\left(\mathrm{Q}_{\mathrm{high}}+\right.$ erys; $n=4$ ). The values are means of all perfusions performed in that category.

used in perfusions with a low and a high flow rate of medium through the oxygenator (Figure 5.3). Addition of perfluortributylamine or erythrocytes to the medium appeared to prevent hepatocellular damage caused by the perfusion; however, sinusoids were more dilated using perfluortributylamine (Figure 5.4).

\section{Metabolic activity}

DMAc, which was used as a substrate to study biotransformation activity of the liver, was added to the medium just before the start of the perfusion. After perfusion was started. DMAc disappeared from the medium with a first-order 'elimination' rate constant of $0.019 \pm 0.008 \mathrm{~min}^{-1}(\mathrm{n}=3)$ without and $0.022 \pm$ 0.014 min $^{-1}(n=4)$ with addition of erythrocytes (difference not statistically significant by Wilcoxon two sample test; Figure 5.5). The disappearance of DMAc from the medium was clearly "liver dependent" as the DMAc concentration remained constant during 'perfusions' without livers, regardless of the presence of erythrocytes. Monomethylacetamide, a metabolite of DMAC [2,6] could not be detected in perfusate samples (less than $35 \mu \mathrm{M}$ ). 

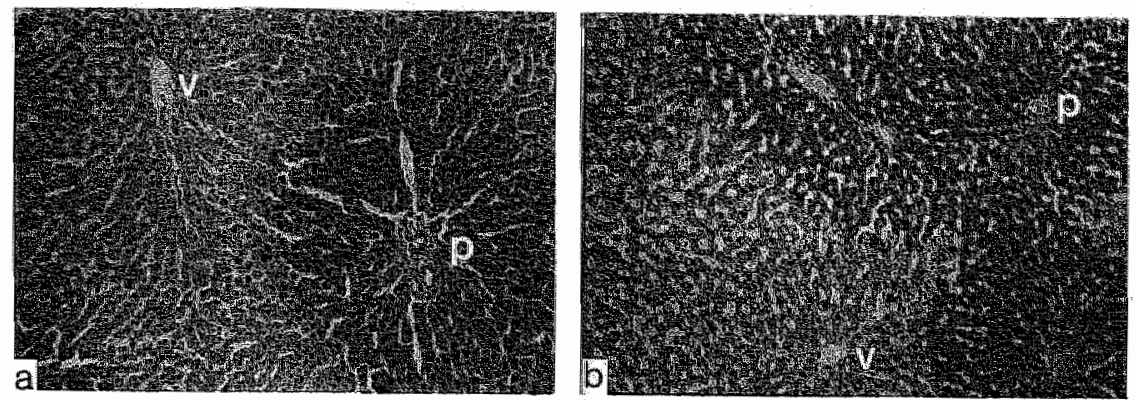

Figure 5.3: Histological evaluation of livers perfused with a 1-hr recirculating perfusion after haematoxilineosin staining. Medium was composed of standard medium and (a) an oxygenator medium flow of $92 \mathrm{ml} \cdot \mathrm{min}^{-1}$ or (b) $186 \mathrm{ml} \cdot \mathrm{min}^{-1}$. $\mathrm{P}=$ Afferent terminal (portal) venule; $\mathrm{V}=$ efferent terminal (hepatic) venule. Note the extensive necrosis in the central lobular areas (Rappaport areas 2 and 3). Original magnification $\times 160$.
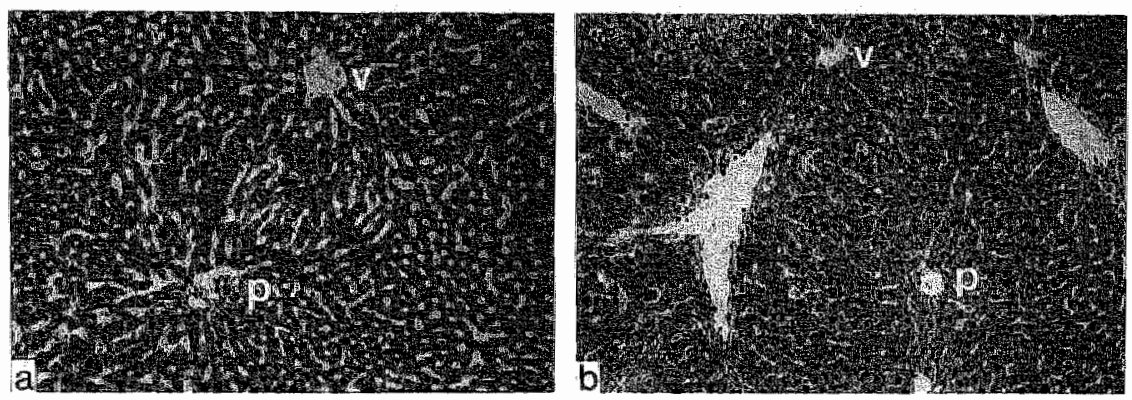

Figure 5.4: Histological evaluation of livers perfused with a 1-hr recirculating perfusion after haematoxilin-eosin staining. Medium was composed of standard medium and (a) perfluortributylamine ( $\mathrm{FC43}$ ) with an oxygenator medium flow of $92 \mathrm{ml} \cdot \min ^{-1}$ or (b) erythrocytes $\left(\mathrm{Hb}=4 \mathrm{~g} \cdot \mathrm{l}^{-1}\right)$, and ari oxygemator nediun How of $186 \mathrm{ml} \cdot \mathrm{min}^{-1} . \mathrm{P}=$ Afferent terminal (portal) venule; $\mathrm{V}=$ efferent terminal (hepatic) venule. Note the dilated simusoids in the FC43-perfused liver and the healthy appearance of the erythrocyte-perfused liver. Original magnification $\times 160$.

\section{Discussion}

The isolated perfused rat liver technique is often used to study the metabolism of xenobiotics because the metabolism is not influenced by external, hormonal or neural factors, and metabolic activity of other organs than the liver is ex- 


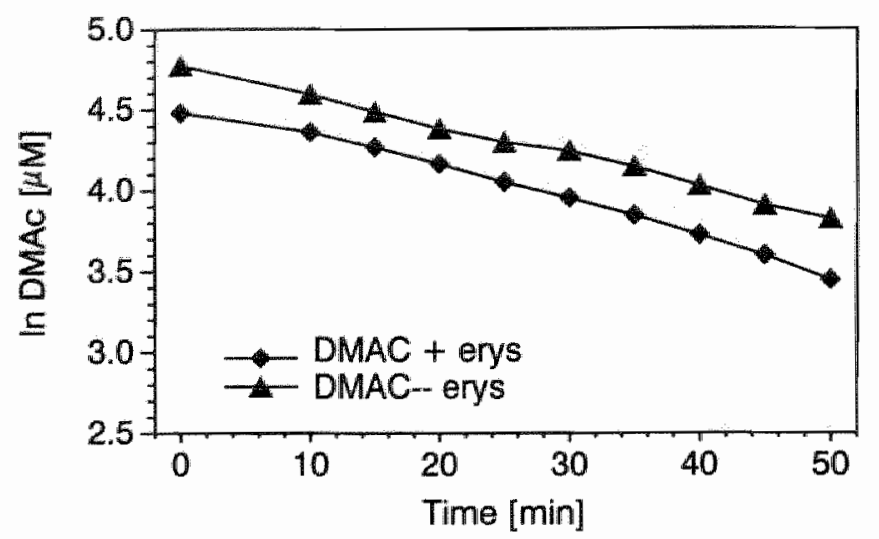

Figure 5.5: Time course of dimethylacetamide (DMAc) concentration during perfusions with standard medium and an oxygenator medium flow of $186 \mathrm{ml} \cdot \mathrm{min}^{-1}$ (DMAc-erys: $\mathbf{n}, \mathbf{n}=3$ ), or perfusions with the same medium supplied with erythrocytes $\left(\mathrm{Hb}=4 \mathrm{~g} \cdot \mathrm{l}^{-1}\right.$ ) and an oxygenator medium flow of $186 \mathrm{ml} \cdot \mathrm{min}^{-1}$ (DMActerys; $n=4$ ). DMAc concentrations were means of all perfusions performed in that category.

cluded. The type and quantity of metabolites formed is influenced substantially by the composition of perfusate, degree of oxygenation, temperature, perfusate flow rate, concentration of the substrate tested and the presence of inhibitors or inducers [14]. Medium composition is often rather artificial compared with that of normal blood, which may have a considerable influence on the metabolic activities of the liver [10]. Inadequate oxygenation of the medium may reduce substrate extraction, especially for downstream hepatocytes [15]. Thurman and Scholz [20] reported that oxygen uptake in perfused livers from normal and phenobarbitaltreated rats was $100-125 \mu$ moles $\cdot \mathrm{g}^{-1} \cdot \mathrm{h}^{-1}$. Using phenobarbital-pretreated starved rats, oxygen consumption of the liwer was found to increase by $16 \mu \mathrm{moles} \cdot \mathrm{g}^{-1} \cdot \mathrm{h}^{-1}$ when the monooxygenase substrate aminopyrine was added to the medium.

Bile formation, as an indicator of the functionality of the secretory mechanisms, is often used as a parameter to evaluate liver integrity. Normal bile secretion rate is of the order of $0.9 \mathrm{mg} \cdot \mathrm{min}^{-1} \cdot \mathrm{g}^{-1}[7]$ and is impaired by hepatotoxic compounds such as chloroform [4]. Bile formation requires the expenditure of metabolic energy for the performance of osmotic work, and depends on many factors such as perfusate How rate and temperature [3]. In the present study the low rate of bile production at the beginning of the perfusions can be explained by sub-optimal conditions (e.g. medium temperature) caused by technical adjustments. Bile production rate in our perfusions with standard medium at high flow rate through the artificial lungs was about equal to that reported by Krell et al. [7]. When standard medium at a low flow rate over the oxygenators was used, the amount 
of bile secreted was only about half this value. The addition of oxygen carriers increased bile production. The flow rate through the liver did not vary between experiments.

ASAT activities after one hour perfusions using medium containing diluted rat blood were reported to be in the range of $60-80 \mathrm{U} \cdot \mathrm{l}^{-1}[1,9]$. In the present study ASAT activity was about $10 \mathrm{U} \cdot 1^{-1}$ after a 1 hour perfusion with medium containing erythrocytes or perfluortributylamine, indicating that cell membranes were still intact. Without these additions much higher ASAT leakage was found (Figure 5.2).

Histological examination of the liver after perfusions with erythrocytes or perfluortributylamine revealed less impairment than after perfusions without additions. These results were in agreement with those of Schmucker and Curtis [18] who reported extensive injury of the sinusoidal endothelium and vacuolization in centrilobular parenchymal cells caused by oxygen deficiency using standard medium. Increasing the flow through the liver from 15 to $72 \mathrm{ml} \cdot \mathrm{min}^{-1}$ in the erythrocyte-free system still resulted in injured centrilobular cells; addition of erythrocytes to the Krebs medium solved this problem. Perfluortributylamine caused 'ballooning' of sinusoids; this phenomenon was also reported by Pang [14]].

Oxygen supply is not only important to maintain liver function, but also for metabolic activity. As the pericentral oxygen concentration is lower than the periportal oxygen concentration, and oxidative reactions of substrates requiring oxygen are often mediated by the cytochrome P-450 monooxygenation system, which is concentrated in downstream hepatocytes [15], metabolism of substrates could be impaired when oxygen supply is deficient. Theophylline, for example, is catabolized chiefly by oxygen-dependent enzymes [12]; when the oxygen tension in the medium decreased, theophylline clearance was also found to be decreased. In spite of a better oxygen supply to the liver when erythrocytes were used, no significant differences in DMAc metabolism were found in the present study between erythrocyte-amended medium $(n=4)$ and standard medium $(n=3)$. The extraction ratio for DMAc was $0.0579 \pm 0.030$ when no erythrocytes were added, and $0.073 \pm 0.041$ when erythrocytes were added to the medium.

On the basis of our results we may conclude that oxygen supply to the liver is critical for liver function. Standard medium and a low flow rate of medium through the oxygenator $\left(92 \mathrm{ml} \cdot \mathrm{min}^{-1}\right)$ leads to impaired bile secretion, increased enzyme activity in the perfusate and microscopically visible damage. When the flow rate of medium through the oxygenator was increased $\left(186 \mathrm{ml} \cdot \mathrm{min}^{-1}\right)$, bile secretion increased but perfusate enzyme activity remained high and microscopy still showed heavily damaged cells in the central lobular region. Both bile secretion and perfusate enzyme activity were closer to normal when oxygen carriers were used. Fluorocarbons (e.g. perfluortributylamine) may act as monooxygenation uncouplers [21] and are phagocytized by Kupffer cells [17], a process that 
was reported to cause depression of cytochrome P-450 levels and a decrease in the related drug biotransformation [16]. In our opinion, therefore, the use of perfluortributylamine is not advisable in biotransformation studies. In conclusion, erythrocytes added to standard medium lead to good bile production and low perfusate enzyme activities. Elimination of DMAc, however, was not significantly improved using medium supplied with erythrocytes.

Acknowledgements The authors wish to thank the Red Cross Blood Bank, Zuid-Limburg for collecting the blood samples, the Department of Pathology from the Academic Hospital, Maastricht for the preparation of histological sections and Professor Dr J.W. Arends for the evaluation of the histological sections, A.J.P. van Bommel, A.L.A. Sesink and G. Koppen for their technical support, H.M.J. Roelofs, Department of Gastroenterology (University of Nijmegen) and Dr G.M.M. Groothuis, Department of Pharmacology and Therapeutics (University of Gromingen; Head: Professor Dr D.K.F. Meijer), for their support during the setting-up of liver perfusions and valuable discussions. 


\section{Bibliography}

(1) Bartosek, I, Guaitani, A and Garattini, S. (1972) Long-Term Perfusion of Isolated Rat Liver. Pharmacology 8, 244-258.

[2] Borm, P.J.A., De Jong, L. and Vliegen, A. (1987) Environmental and Biological Monitoring of Workers Occupationally Exposed to Dimethylacetamide. I. Occup. Med. 29, 898-903.

[3] Brauer, R.W. (1957) Liver. Anru. Rev. Physiol. 18, 253-278.

(4) Brauer, R.W. Leong, G.F. and Holloway, R.J. (1960) Liver injury in isolated perfused rat liver preparation exposed to chloroform. Am. J. Physiol. 200, 548550.

[5] Katsuyuki, N., Jamieson, D., Oshino, N. and Chance, B. (1976) Oxygen Toxicity in the Perfused Rat Liver and Lung under Hyperbaric Conditions. Biochem. J. $160,343-355$.

[6] Kennedy, G.L. and Pruett, J.W. (1989) Biologic Monitoring for Dinethylacetamide: Measurement for 4 Consecutive Weeks in a Workplace. J. Occup. Med. 31, $47-50$.

[7] Krell, H., Jäschke, H., Höke, H. and Pfaff, E. (1984) Bile secretion in HemoglobinFree perfused Rat Liver. Z. Physiol. Chem. 365, 1115-1122.

[8] Kreutzer, H.J.H. Klinischmchemisch laboratorium onderzoek, Elsevier, Amsterdam, 1982, pp. 170-172.

[9] Květina, J. and Guaitani, A. (1969) A Versatile Method for the in vitro Per. fusion of Isolated Organs of Rats and Mice with Particular Reference to Liver. Pharmacology 2, 65-81.

[10] Lindros, K.O. The Once-Through Perfusion Technique: Advantages and Limitations, in: Lundquist, F. and Tygstrup, N. (eds.) Regulation of Hepatic Metabolism, Academic Press, Munksgaard, Copenhaken, Alfred Benzoin Symposium VI, 1974, pp. $778-789$.

[11] Meijer, D.K.F. Keulemans, K. and Mulder, G.J. (1981) Isolated Perfused Rat Liver Technique. Methods Enzymol. 77, 81-94.

[12] Miller, R. and Oliver, $\mathbb{1}$. F. (1986) The influence of oxygen tension on theophylline clearance in the rat isolated perfused liver. J. Pharm. Pharmacol. $38,236-238$.

[13] Moss, D.W., Henderson, R. and Kachmar, J.F. Enzymes, in Tietz, N.W. (ed.) Textbook of Clinical Chemistry, W.B. Saunders Company, Philadelphia, 1986, pp. 669677 .

[14] Pang, K.S. Liver Perfusion Studies in Drug Metabolism and Drug Toxicity, in: Mitchell, J.R. and Horning, M.G. (eds.) Drug Metabolism and Drug Toxicity; Raven Press, New York, 1984, pp. 331-352.

[15] Pang, K.S., Lee, W.F., Cherry, W.F., Yuen, V., Accaputo, J, Fayz, S., Schwab, A.J. and Goresky, C.A. (1988) Effects of Perfusate Flow Rate on Measured Blood Volume, Disse Space, Intracellular Water Space, and Drug Extraction in the Perfused Rat Liver Preparation: Characterization by the Multiple Indicator Dilution Technique J. Pharmacokinet. Biopharm. 16,595-632.

[16] Peterson, T.C. and Renton, K.W. (1986) Kupffer cell factor mediated depression of hepatic parenchymal cell cytochrome P-450. Biochem. Pharmacol. 35, 1491-1497. 
[17] Ruigrok, T.J.C. and Elbets, P.F. (1970) Ultrastructural changes after perfusion of isolated rat liver with a fluorocarbon emulsion as a substitute for erythrocytes. Cytobiology 2, 315-325.

[18] Schmucker, D.L. and Curtis, J.C. (1974) A Correllated Study of the Fine Structure and Physiology of the Perfused Rat Liver. Lab. Invest. 30, 201-212.

[19] Sies, H. (1977) Oxygen Gradients During Hypoxic Steady States in liver. Urate Oxidase and Cytochrome Oxidase as Intracellular $\mathrm{O}_{2}$ Indicators. Z. Physiol Chem. 358, 1021-1032.

[20] Thurman, R.G. and Scholz, R. (1969) Mixed Function Oxidation in Perfused Rat Liwer. The ffect of Aminopyrin on Oxygen Uptake. Eur. đ. Biochem. 10, 459-467.

[21] Ullrich, $V$. and Diehl, $H$. (1971) Uncoupling Monooxygenation and Electron Transport by Fluorocarbons in Liver Microsomes. Eur. J. Biochem, 20, 509-512. 
Chapter 6

\section{Oxidative effects in human erythrocytes caused by some oximes and hydroxylamine}

Nicole G.M. Palmen, Astrid H.J.M.G. Feijts and Chris T.A. Evelo

Department of Pharmacology, Toxicology section, University of Limburg, PO Box 616, 6200 MD Maastricht, The Netherlands.

Subrnitted for publication 


\section{Summary}

Both oximes and hydroxylamine (HYAM) are compounds with known oxidative capacity. We tested in vitro whether acetaldoxime (AAO), cyclohexanone oxime (CHO), methyl-ethyl ketoxime (MEKO) or hydroxylamine (HYAM) affect hemoglobin oxidation (into $\mathrm{HbFe}^{3+}$ ), thiobarbituric acid reactive substances (TBARS) formation, and glutathione (GT) depletion in human hemolysate, erythrocytes or blood. All these parameters are known to be related to oxidative stress. Glutathione S-transferase (GST) activity was measured as it may be affected by oxygen radicals. All three oximes caused a low degree of $\mathrm{HbFe}^{3+}$ accumulation in erythrocytes. This was higher in hemolysates indicating that membrane transport may be limiting. HbFe $e^{3+}$ accumulation for the oximes was lower than for HYAM. AAO and HYAM caused TBARS formation in blood. For HYAM this was expected as free radicals are known to be generated during $\mathrm{HbFe}^{3}$ * formation. Free radical generation by $A A O$ and HYAM in erythrocytes was confirmed by the inhibition of GST. For the other two oximes (CHO and MEKO) some special effects were found CHO did inhibit erythrocyte GST while it did not cause TBARS formation. MEKO was the least potent oxime as it caused no TBARS formation, little $\mathrm{HbFe}^{3+}$ accumulation and little GST inhibition in erythrocytes. However, GT depletion was more pronounced than for the other oximes, indicating that ghutathione conjugation occurs. TBARS formation, GT depletion and GST modulation caused by the oximes and HYAM were also tested in rat hepatocytes. However, no effects were found in lnepatocytes. This suggests that a factor present in erythrocytes is necessary for free radical formation.

Studies with suggested metabolites of the oximes (i.e. cyclohexanone, acetaldehyde or methyl-ethyl ketone) and addition of rat liver preparations to the erythrocyte incubations with oximes, suggest that metabolism is not a limiting factor in erythrotoxicity.

\section{Introduction}

Oximes are compounds containing the common structure $\mathrm{HO}-\mathrm{N}=\mathrm{C}$ and are there fore structurally related to hydroxylamine ( $\mathrm{HO}-\mathrm{NH}_{2}$ : HYAM). Among other applications, oximes are used in paints and enamels. Cyclohexanone oxime (CHO) is used in the production of caprolactam, which in turn is utilized in the production of nylon. Some oximes are known hematotoxic agents. Subacute and subchronic oral exposure of rats to CHO causes erythrocyte deformations (Howell Jolly bodies), hemoglobin oxidation, a decreased number of erythrocytes, a decrease in hemoglobin content and a lower hematocrit, with an accompanying. increase in circulating reticulocytes and nucleated erythrocytes $[13,21]$. Chronic exposure of rats to methyl-ethyl ketoxime (MEKO) (subcutaneaus injections) causes hypertrophy of the spleen, and a decrease in erythrocyte count and hemoglobin content [34]. These studies indicate that the primary target organ in wivo is the erythrocyte, causing hemolytic anemia with compensatory extramedullary 
erythropoiesis, as well as hemosiderosis in the spleen. It is not well understood whether the hematotoxic effects associated with these oximes are due to the parent compound per se or to their metabolites. There are indications that oximes may be hydrolyzed to yield the corresponding aldehyde or ketone structure plus HYAM $[28,42]$, a compound with strong oxidative capacity with regard to hemoglobin [23].

Due to their abundancy and presence all over the body, erythrocytes can easily become a target for toxic compounds. Under normal conditions, erythrocytes are exposed to oxidant stress caused by the high concentration of molecular oxygen and oxidizing substances released into the blood [22]. In order to offer resistance to these challenges, the erythrocyte contains large amounts of protein and non-protein anti-oxidants. When oxidant stress increases to abnormal levels, damage may occur. Xenobiotics may also increase the oxidant stress by disturbing the anti-oxidant systems, the erythrocyte membrane- or hemoglobin structures [22,51]. Normal physiological (auto)oxidation of hemoglobin is known to lead to superoxide formation [8]. Recently Stolze and Nohl [49] demonstrated liberation of other free radical products as a result of hemoglobin oxidation by hydroxylamine. Since the oxidation of hemoglobin to methemoglobin is a one electron step, radical formation is expected as a general result. This may initiate lipicl peroxidation of the erythrocyte membrane, which can be measured as thiobarbituric acid reactive substances (TBARS) [2]. Free radicals can also oxidise protein and non-protein thiols. Glutathione is the most important non-protein thiol and may be oxidized into GSSG [12]. Peroxidized lipid products may be reduced by glutathione peroxidase or glutathione S-transferase (GST) at the expense of reduced glutathione generating GSSG [18]. When the reductive capacity of the cell is insufficient, GSSG can be released from the cell $[12,33]$. In addition to excretion of GSSG, the erythrocyte glutathione content may also be depleted by mixed disulfide formation [24]. Erythrocyte GST is a GST- $\pi$ class enzyme [5], which is known to be inactivated by free radicals $[14,15,16,38,45,46]$.

The aim of the present study was to test whether the oximes acetaldoxime (AAO), CHO and MEKO as such are able to generate oxidative effects like hemoglobin oxidation, TBARS formation and glutathione depletion or GST activity changes in human erythrocytes. To study whether metabolites of the oximes are able to generate these effects, two distinct approaches were used. On one hand, $\mathrm{AA}, \mathrm{CH}$, MEK and HYAM, which are suggested metabolites of AAO, CHO and MEKO were tested. On the other hand, oximes were also tested in a combined assay of erythrocytes and microsomes or hepatocytes, and in an isolated rat liver perfusion in which human erythrocytes were added to the medium. We tested whether metabolites generated by these metabolizing systems were able to generate oxidative effects in erythrocytes and/or hepatocytes. 


\section{Materials and Methods}

\section{Chemicals}

Hydroxylamine (HYAM (ACS reagent); 99.1\%: CAS: 5470-11-1) and all nonspecified enzymes and cofactors were from Sigma (St. Louis, USA), cyclohexanone $(\mathrm{CH} ;>99.5 \%$; CAS: 108-94-1) and methyl-ethyl ketone (MEK; >99.5\%; CAS: 78-93-3) were from Fluka Chemie (Buchs, Switserland), acetaldehyde (AA; $>99 \%$; CAS: 1632-89-9) was from Merck (Schuchardt, Germany). Cyclohexanone oxime (CHO; $>99.5 \%$; CAS: 100-64-1), methyl-ethyl ketoxime (MEKO; >99\%; CAS: 96-29-7) and acetaldoxime (AAO; 99.7\%; CAS: 107-29-9) were a gift from DSM special products (Geleen, The Netherlands). Collagenase B (EC 3.4.24.3; from Clostridium histolyticum; Lot: 12086224-05) was obtained from Boehringer (Mannheim, Germany). Water was demineralized and microfiltrated before use. All other chemicals were of analytical grade quality.

\section{Preparation of erythrocytes and lysate}

Human blood was obtained from the Red Cross Blood Bank "Zuid Limburg" where it had been collected in sterile vacuum EDTA vessels and kept overnight at $4^{\circ} \mathrm{C}$. Erythrocytes from at least 4 persons were pooled, washed three times using saline and diluted in Krebs-Henseleit buffer ( $\mathrm{pH} \mathrm{7.4)}$ to a hemoglobin (Hb) concentration of $27 \mathrm{~g}^{-1} \mathrm{I}^{-1}$ in incubations with transwells ${ }^{T M}$ (Costar, Cambridge) or washed erythrocytes, and $23 \mathrm{~g} \cdot \mathrm{l}^{-1}$ in incubations with microsomes. For isolated liver perfusions, erythrocytes were diluted in Krebs-Ringer buffer solution ( $\mathrm{pH} 7.4$ ) to a $\mathrm{Hb}$ concentration of $4 \mathrm{~g} \cdot \mathrm{l}^{-1}[40]$. Hb concentrations were determined according to van Kampen and Zijlstra [31]. Lysate was prepared from packed erythrocytes by dilution with ice-cold water $(1: 2)$.

\section{Preparation of microsomes and isolation of hepatocytes}

Male Brown Norway rats $(\mathrm{BN} / \mathrm{M})$ of approximately $250 \mathrm{~g}$ were used. Housing was as previously described [40]. Animals were anesthetized with pentobarbital (Narcovet ${ }^{T M} ; 0.1 \mathrm{ml} / 100 \mathrm{~g}$ rat). Microsomes were prepared as described previously [41] Microsomal cytochrome P-450 content was determined in duplicate using the dithionite-difference method according to Rutten et al. [43], and was found to be $0.78-0.85$ nanomoles $\mathrm{mg}^{-1}$ protein. The procedure for the isolation of hepatocytes was based on the methods described by Berry and Friend [3] and by Seglen [44]. A calcium and magnesium free buffer solution ( $100 \mathrm{mM} \mathrm{NaCl}, 7 \mathrm{mM}$ $\mathrm{KCl}, 39 \mathrm{mM}$ HEPES and $15 \mathrm{mM} \mathrm{NaH} \mathrm{PO}_{4}$ (pH adjusted to 7.4 with $\mathrm{NaOH}$ )) was perfused $\left(30 \mathrm{ml} \cdot \mathrm{min}^{-1}\right)$ in a single pass way through the isolated rat liver during 20 minutes. Next, a collagenase containing buffer $\left(0.05 \mathrm{~g} \cdot 100 \mathrm{ml}^{-1}\right)$, composed of $67 \mathrm{mM} \mathrm{NaCl}, 7 \mathrm{mM} \mathrm{KCl}, 100 \mathrm{mM} \mathrm{HEPES}$ and $5 \mathrm{mM} \mathrm{CaCl} \cdot 2 \mathrm{H}_{2} \mathrm{O}$ (pH adjusted to 7.4 with $\mathrm{NaOH}$ ), was perfused in a recirculating way during 5 minutes. 
Hepatocytes were isolated from the liver, filtrated $(100 \mu \mathrm{M})$ and washed three times in a Krebs-Henseleit buffer ( $\mathrm{pH} 7.4 ; 4$ minutes, centrifuge force: $25 \times \mathrm{g}$ ). The viability of the cells (over $85 \%$ ) was determined with the Trypan-blue exclusion test $[29]$.

\section{Incubations}

Incubations with whole blood or hemolysate

Both in incubations with whole blood and in incubations with lysate (final volume $3.5 \mathrm{ml}$ ), $350 \mu$ test compound (i.e. CHO, AAO, MEKO, HYAM, CH, AA and MEK), diluted in Krebs-Henseleit buffer or in water respectively (final concentrations between 1 and $7 \mathrm{mM}$ ), was added and incubated for 1 hour at $37^{\circ} \mathrm{C}$ in a shaking water bath ( 80 revolutions per minute). In hemolysate, $\mathrm{HbFe}^{3+} \mathrm{ac}-$ cumulation, TBARS formation, activity changes of GST, and depletion of the sum of both reduced and oxidized glutathione (GT) were measured to test CHO, AAO, MEKO, HYAM, CH, AA and MEK. In blood, TBARS formation was tested for all compounds; $\mathrm{HbFe}^{3+}$ accumulation, GST activity changes and GT depletion were tested only for $\mathrm{CH}$, AA or MEK. Each day the incubations with whole blood or hemolysate were performed in triplicate. The experiments were performed three times on different days.

\section{Incubations with erythrocytes}

The incubations of erythrocytes were performed similar to the co-incubations of erythrocytes and hepatocytes (see below), replacing hepatocytes by KrebsHenseleit buffer. CHO, AAO and MEKO (final concentrations 1,2 and $3 \mathrm{mM}$ ) and HYAM (final concentration $3 \mathrm{mM}$ ) were tested. In erythrocytes $\mathrm{HbFe}^{3+}$ accumulation, GST activity changes and GT depletion caused by CHO, AAO, MEKO or HYAM were measured. The incubations were tested in duplicate each day and were performed three times on different days.

\section{Co-incubation of erythrocytes and microsomes}

Incubations using washed erythrocytes ( $\mathrm{Hb} 23 \mathrm{~g}^{-1}$ ) plus microsomes were performed as described previously [41]. The erythrocytes were washed three times with saline after an incubation period of 1 hour. AAO and MEKO concentrations between 1 and $7 \mathrm{mM}$, and $\mathrm{CHO}$ concentrations of 3.1 and $7.8 \mathrm{mM}$ were tested. $\mathrm{HbFe}^{3+}$ accumulation, GST activity changes and GT depletion were measured. Each day the incubations were performed in triplicate. The experiments were performed twice on different days. 


\section{Incubations with hepatocytes}

Hepatocytes were diluted to a cell concentration of $6.6 \times 10^{6}$ cells $\mathrm{ml}^{-1}$ with KrebsHenseleit buffer. Hepatocytes $(1 \mathrm{ml})$ were incubated in triplicate with $\mathrm{CHO}, \mathrm{AAO}$, MEKO or HYAM $(0.5 \mathrm{ml}$, final concentration between 1 and $7 \mathrm{mM})$ or $\mathrm{CH}, \mathrm{AA}$ or MEK (0.5 ml, final concentration $7 \mathrm{mM})$, for 1 hour at $37^{\circ} \mathrm{C}$ in a shaking water bath ( 80 revolutions per minute). GST activity changes, GT depletion and TBARS formation were measured. In each experiment all incubations were performed in triplicate. The experiments were performed three times on different days.

\section{Co-incubation of erythrocytes and hepatocytes}

Co-incubations of erythrocytes and hepatocytes were performed in transwells (a well in which a transwell, containing a porous $0.4 \mu \mathrm{m}$ membrane, can be placed (Costar, Cambridge, England)) Erythrocytes (2.6 $\mathrm{ml}, \mathrm{Hb} 27 \mathrm{~g} \cdot \mathrm{l}^{-1}$ ) were pipetted into the well, and the hepatocytes $\left(1 \mathrm{ml}, 6.6 \times 10^{6}\right.$ cells $\left.\cdot \mathrm{ml}^{-1}\right)$ plus CHO, AAO or $\mathrm{MEKO}$ (final concentrations between 1 and $3 \mathrm{mM}$ ) or HYAM (final concentration $3 \mathrm{mM}$ ) were added to the transwell. $\mathrm{HbFe}^{3+}$ accumulation, GST activity changes and GT depletion were measured. Each day the experiment was performed in duplicate and repeated three times on different days.

To optimize cellular contact between hepatocytes and erythrocytes, co-incubations were also performed in which the two cell types were mixed. Cellular volume and concentrations were identical to those of the transwells. CHO, AAO, MEKO or HYAM concentrations were between 1 and $3 \mathrm{mM}$. After a one hour incubation period, erythrocytes were separated from the hepatocytes by density centrifugation using isotonic Percoll (gravity $1.08 \mathrm{~g}^{-l^{-1}}, 4^{\circ} \mathrm{C}$, centrifuge force $400 \times \mathrm{g}$ ). Erythrocytes were sampled and washed three times with saline (15 mM). In a single experiment, each test concentration was tested in triplicate.

\section{Isolated rat liver perfusions}

Liver perfusions were performed as described previously [40]. Medium was composed of Krebs-Ringer buffer solution ( $\mathrm{pH} 7.4$ ) containing washed human erythrocytes (Hb concentration $4 \mathrm{~g}^{-1}$ plus $0.1 \%$ bovine serum albumin to prevent cell lysis). CHO, AAO or MEKO (final concentration $3 \mathrm{mM}$ ) were added to the perfusion medium before the start of the perfusion $(n=1)$. In addition, liver perfusions without test compound were performed $(n=2)$. Medium flow was kept constant for the perfusion. Bile samples were taken every 10 minutes. Mean bile production was $0.6(\mathrm{SE}=0.1) \mathrm{mg} \cdot \mathrm{min}^{-1} \cdot \mathrm{g}^{-1}$ liver. Perfusate samples were taken before the start of the perfusion and every 10 minutes thereafter during 1 hour. $\mathrm{HbFe}^{3+}$ accumulation, GST activity changes and GT depletion were measured. Liver damage was assessed through lactate dehydrogenase (LDH) activity (EC 1.1.1.27) in the 
perfusate samples, using NADH consumption during pyruvate transformation to lactate [37]. Albumin was added to these samples prior to storage $\left(-70^{\circ} \mathrm{C}\right.$; final concentration $3 \%$ ). Mean lactate dehydrogenase leakage was never higher than $100 \mathrm{U} \cdot \mathrm{I}^{-1}$. Bile production and lactate dehydrogenase leakage indicate that liver viability was good [40].

\section{Analytical procedures}

Percentage of methemoglobin $\left(\mathrm{HbFe}^{3+}\right)$

Samples were prepared according to the following procedures: a) In incubations with whole blood, $500 \mu$ blood was centrifuged $(2000 \times \mathrm{g}, 5 \mathrm{~min}$.). After removing the plasma, erythrocytes were lysed with $2 \mathrm{ml}$ ice cold water and kept on ice during 15 minutes. b) Washed erythrocytes $\left(1.2 \mathrm{ml}\right.$, Hb $27 \mathrm{~g} \cdot \mathrm{l}^{-1}$ or $\left.23 \mathrm{~g} \cdot \mathrm{l}^{-1}\right)$ were concentrated by centrifugation ( $2000 \times \mathrm{g}, 5 \mathrm{~min}$.). After removing the supernatant, the erythrocytes were lysed with $2.25 \mathrm{ml}$ ice cold water and kept on ice during 15 minutes. Perfusate samples derived from liver perfusions $\left(2.7 \mathrm{ml}, \mathrm{Hb} 4 \mathrm{gl}^{-1}\right)$ were also centrifuged $(2000 \times \mathrm{g}, 5 \mathrm{~min}$.). After removing the supernatant, the erythrocytes were lysed with $750 \mu \mathrm{l}$ ice cold water. c) Lysate $(750 \mu l)$ was diluted with $1.5 \mathrm{ml}$ water.

After the addition of potassium phosphate buffer ( $150 \mathrm{mM}, \mathrm{pH} 6.6,1: 1 \mathrm{v} / \mathrm{v}$ ) to the samples, the cellular debris was removed by centrifugation $(2000 \times \mathrm{g}, 3$ min.). The percentage $\mathrm{HbFe}^{3+}$ present in hemolysates was calculated from the absorbance change after addition of KCN compared to the same change in a sample fully converted to the $\mathrm{HbFe}^{3+}$ form by the addition of $\mathrm{K}_{3} \mathrm{Fe}\left(\mathrm{CN}_{26}\right.$ as described by Betke et al. [4].

\section{Thiobarbituric acid reactive substances (TBARS)}

Cells were removed from the incubations by centrifugation $(2000 \times \mathrm{g}$ for blood cells and $25 \times \mathrm{g}$ for hepatocytes, $5 \mathrm{~min}$.). TCA (10\%) was added to the supernatants $(1: 1 \mathrm{v} / \mathrm{v})$. After centrifugation of the denaturated samples $(2000 \times \mathrm{g}, 5$ min.), the supernatant was incubated with thiobarbituric acid (TBA; $1 \%, 1: 1$ $\mathrm{v} / \mathrm{v})$ during 15 minutes $\left(100^{\circ} \mathrm{C}\right)$ and cooled immediately thereafter $\left(10^{\circ} \mathrm{C}\right)$. The malondialdehyde-thiobarbituric acid complex was extracted into 1-butanol and the difference in absorption between the measurements at $\lambda=535 \mathrm{~nm}$ and $\lambda=590$ nm was determined. TBARS concentrations were calculated using a molar extinction coefficient of $1.56 \times 10^{5} 1 \cdot \mathrm{mol}^{-1} \mathrm{~cm}^{-1}[47,48]$. As AAO and AA also react with TBA, incubations with these compounds were corrected for this back-ground reactivity. 


\section{Glutathione concentrations}

Samples derived from whole blood, hemolysate or hepatocytes were denaturated with TCA $(8 \%, 1: 1 \mathrm{v} / \mathrm{v})$ and centrifuged $(10000 \times \mathrm{g}, 5 \mathrm{~min})$. The supernatant was diluted with $100 \mathrm{mM}$ sodium/potassium phosphate buffer $(\mathrm{pH} 7.4,1.8 \mathrm{v} / \mathrm{v}$ ) and stored $\left(-70^{\circ} \mathrm{C}\right)$ until determination. Erythrocytes from the erythrocyte incubations $(150 \mu \mathrm{l})$ and from the perfusion experiments $(400 \mu \mathrm{l})$ were concentrated by centrifugation. $25 \mu$ TCA $(8 \%)$ was added to the pellet and the samples were frozen until determination $\left(-70^{\circ} \mathrm{C}\right)$. After thawing, the samples were diluted 50 and 25 times respectively, with sodium/potassium phosphate buffer $(100 \mathrm{mM})$, and centrifuged ( $10000 \times \mathrm{g}, 3 \mathrm{~min})$. GT was determined with the cyclic oxidation reduction method essentially as described by Anderson [1].

\section{Glutathione S-transferase (GST) activity}

Blood cells and hepatocytes were lysed ( $15 \mathrm{~min}$.) by addition of four volumes ice cold water containing $1.4 \mathrm{mM}$ dithiothreitol (DTT). Erythrocytes from the purified erythrocyte and co-incubation experiments were first concentrated by centrifugation $(2000 \times \mathrm{g}, 5 \mathrm{~min}$.) of $1 \mathrm{ml}$ sample. The pellets were diluted with $125 \mu \mathrm{l}$ potassium phosphate buffer $(\mathrm{pH} 6.5)$ so that $\mathrm{Hb}$ concentrations of these samples were comparable with the samples derived from whole blood. Erythrocytes in perfusate samples from liver perfusions (Hb about $4 \mathrm{~g} \cdot \mathrm{l}^{-1}$ ) were concentrated by centrifugation $(2000 \times \mathrm{g}, 5 \mathrm{~min}$.) of $1.3 \mathrm{ml}$ sample. Ice cold water $(450 \mu \mathrm{l}) \mathrm{con}-$ taining $1.4 \mathrm{mM}$ DTT was pipetted on the erythrocyte pellet in order to lyse the erythrocytes. Ion strength of hepatocyte, whole blood and erythrocyte samples were re-established by addition of an equal volume of two fold concentrated phosphate buffered saline containing $1.4 \mathrm{mM}$ DTT. In incubations with lysate, the same procedure was used with concentrated PBS containing 2.8 mM DTT.

Samples were centrifuged $(10000 \times \mathrm{g}, 5 \mathrm{~min})$ and the supernatant was stored until determination $\left(-70^{\circ} \mathrm{C}\right)$. After thawing the samples on ice, the GST activity towards chlorodinitrobenzene (CDNB) was determined as described by Habig and Jakoby [26] with a dual beam spectrophotometer (Perkin and Elmer, Lambda $3 \mathrm{~B})$. Hb concentrations in the samples were determined with the hemoglobin cyanide procedure described by van Kampen and Zij]stra [31].

\section{Protein concentrations}

Hepatocytes were lysed with ice cold water $(1: 6.7 \mathrm{v} / \mathrm{v})$ and frozen until determination $\left(-70^{\circ} \mathrm{C}\right)$. Protein concentrations were determined with the Bio-rad Protein Assay (BIO-RAD laboratories, München, Germany). 


\section{Statistical analysis}

Significance of concentration dependent changes in $\mathrm{HbFe}^{3+}$ accumulation and TBARS formation and changes in GT concentrations or in GST activity, were evaluated using linear regression analysis with a model including day of experiment and concentration of the test compound as explanatory variables. Using this model, we tested whether slopes of parameter changes versus concentration were significant correcting for the day of experiment.

\section{Results}

\section{Hemoglobin oxidation}

$\mathrm{HbFe}^{3+}$ accumulation in hemolysate was linear for all three oximes over the concentration range tested ( $p<0.0001$ in all cases, Figure 6.1). CHO was the strongest oxidant $\left(1.8 \% \mathrm{HbFe}^{3+}\right.$ accumulation for each $\mathrm{mM} \mathrm{CHO}$ added) followed by AAO (1.2\%.m.M ${ }^{-1}$ AAO) and MEKO $\left(0.5 \% \cdot \mathrm{mM}^{-1}\right.$ MEKO). HYAM also caused a concentration dependent $\mathrm{HbFe}^{3+}$ accumulation $(p<0.0001)$ and was much more potent than the oximes since hemoglobin was almost completely oxidized by 7 mM HYAM [16]. The oxo-compounds CH, AA and MEK caused no detectable $\mathrm{HbFe}^{3+}$ accumulation.

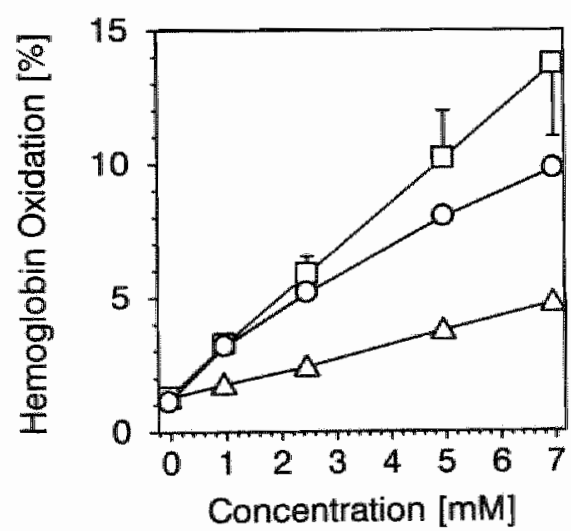

Figure 6.1: Hemoglobin oxidation in hemolysate (incubation period 1 hour; $\left.37^{\circ} \mathrm{C}\right)$. The effect of $\square \mathrm{CHO}, O \mathrm{AAO}$ and $\triangle$ MEKO was tested.

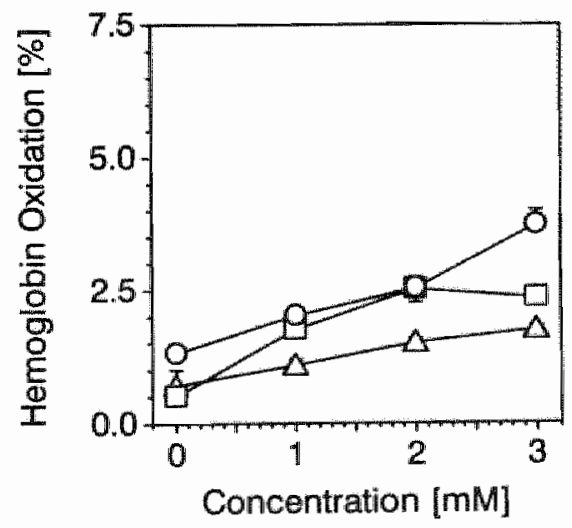

Figure 6.2: Hemoglobin oxidation in erythrocytes (incubation period 1 hour; $\left.37^{\circ} \mathrm{C}\right)$. The effect of $\square \mathrm{CHO}, \mathrm{OAAO}$ and $\triangle \mathrm{MEKO}$ was tested.

In incubations with erythrocytes and oximes (Figure 6.2), $\mathrm{HbFe}^{3+}$ accumulation was concentration dependent $(p<0.01$ in all cases), but was lower than in hemolysates. When concentrations up to $2 \mathrm{mM}$ were tested, CHO was the most 


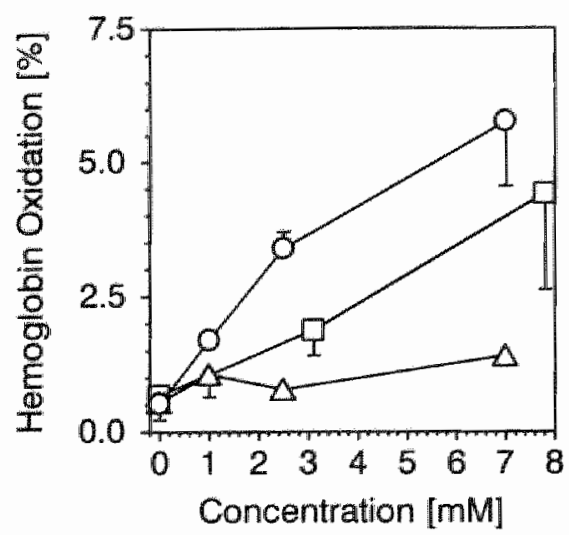

Figure 6.3: Hemoglobin oxidation in erythrocy tes, after co-incubation of ery throcytes with rat liver microsomes (incubation period 1 hour; $37^{\circ} \mathrm{C}$ ). The effect of $\mathrm{CHO}, \mathrm{AAO}$ and $\triangle \mathrm{MEKO}$ was tested.

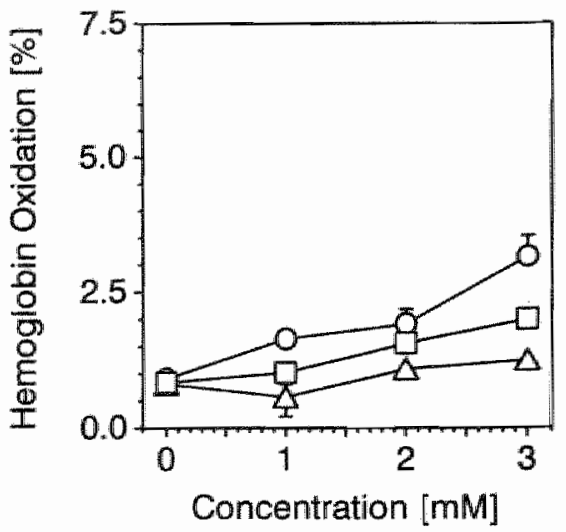

Figure 6.4: Hernoglobin axidation in erythrocytes, after co-incubation of erythrocytes with rat hepatocytes (incubam tion period 1 hour; $37^{\circ} \mathrm{C}$ ). The effect of $\square \mathrm{CHO}, O \mathrm{AAO}$ and $\triangle \mathrm{MEKO}$ was tested.

potent oxime ( $1.0 \% \mathrm{HbFe}^{3+}$ accumulation for each $\mathrm{mM} \mathrm{CHO}$ added), followed by $\mathrm{AAO}\left(0.6 \% \cdot \mathrm{mM}^{-1} \mathrm{AAO}\right)$ and MEKO (0.4\% $\left.\mathrm{mM}^{-1} \mathrm{MEKO}\right)$. In contrast to $\mathrm{AAO}$ and $\mathrm{MEKO}, \mathrm{HbFe}^{3+}$ accumulation by $\mathrm{CHO}$ did not increase at concentrations higher than $2 \mathrm{mM}$. HYAM was again a stronger oxidant than the oximes. However, $\mathrm{HbFe}^{3+}$ accumulation by HYAM in erythrocytes $(88 \%$ at $3 \mathrm{mM}$ ) was higher than in hemolysate $(51 \%$ at $2.5 \mathrm{mM})$. No $\mathrm{HbFe}^{3+}$ was accumulated after the addition of $\mathrm{CH}$ and $\mathrm{MEK}$ to blood. $\mathrm{HbFe}^{3+}$ accumulation caused by $\mathrm{AA}$ in blood was very low $\left(0.05 \% \cdot \mathrm{mM}^{-1} \mathrm{AA}\right)$ but concentration dependent $(p=0.0094)$.

In co-incubations of erythrocytes and microsomes and oximes (Figure 6.3), AAO was the most potent hemoglobin oxidant $\left(0.7 \% \cdot \mathrm{mM}^{-1} \mathrm{AAO} ; p=0.0002\right)$ followed by $\mathrm{CHO}\left(0.5 \% \mathrm{mM}^{-1} \mathrm{CHO} ; p=0.0325\right)$. The effect of MEKO on $\mathrm{HbFe}^{3+}$ accumulation was very limited $\left(0.1 \% \cdot \mathrm{mM}^{-1} \mathrm{MEKO} ; p=0.090\right)$.

When co-incubations of erythrocytes and hepatocytes and oximes were tested using transwells (Figure 6.4), $\mathrm{HbFe}^{3+}$ accumulation was lower compared to the experiments with erythrocytes alone. AAO was the strongest oxidant $\left(0.5 \% \cdot \mathrm{mM}^{-1}\right.$ $\mathrm{AAO})$ followed by $\mathrm{CHO}\left(0.4 \% \cdot \mathrm{mM}^{-1} \mathrm{CHO}\right)$ when concentration up to $2 \mathrm{mM}$ were tested ( $p<0.001$ in both cases). In contrast to incubations with erythrocytes alone, MEKO did not affect $\mathrm{HbFe}^{3+}$ accumulation. HYAM was again a stronger oxidant than the oximes. $\mathrm{HbFe}^{3+}$ accumulation by HYAM $(81.0 \%$ at $3 \mathrm{mM})$ was about the same as in the incubations with erythrocytes alone. Comparable results were obtained from co-incubations in which erythrocytes and hepatocytes were mixed. 
When no oxime was added to an erythrocyte containing medium of an isolated perfused rat liver, no hemoglobin oxidation was found. Addition of CHO, AAO or $\mathrm{MEKO}(3 \mathrm{mM})$ to the perfusion medium caused an increase in $\mathrm{HbFe}^{3+}$ accumulation of $2.9 \%, 3.1 \%$ and $0.9 \%$ respectively, one hour after the start of the perfusion.

\section{Lipid peroxidation}

In incubations with hemolysate (Figure 6.5), TBARS formation was highest for AAO (221 nM (nano moles.1 1 $^{-1}$ TBARS for each mM AAO added), followed by $A A$, which is a possible metabolite of AAO (147 nM TBARS.mM-1 AA) ( $p<0.001$ in both cases). TBARS formation induced by HYAM was much lower than that caused by AAO and was maximal at $2.5 \mathrm{mM}$ HYAM ( $241 \mathrm{nM} ; \mathrm{SE}=25$ ). CHO, MEKO, CH and MEK induced no TBARS formation in hemolysate.

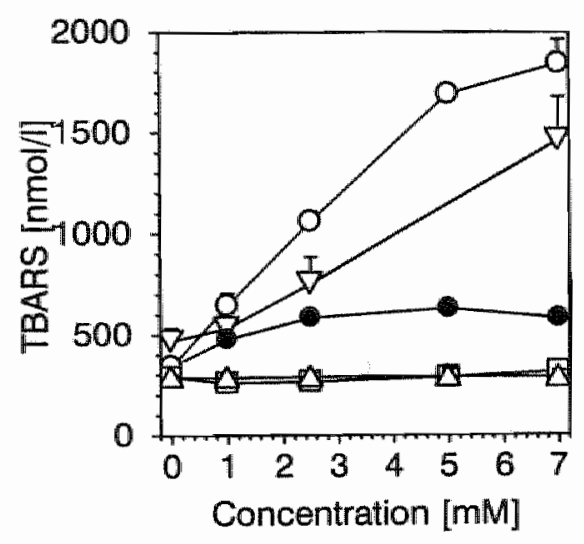

Figure 6.5: Formation of thiobarbituric acid reactive substances in hemolysate (incubation period 1 hour; $37^{\circ} \mathrm{C}$ ). The effect of of $\square$ CHO, O AAO, $\triangle$ MEKO, - HYAM and $\nabla$ AA was tested.

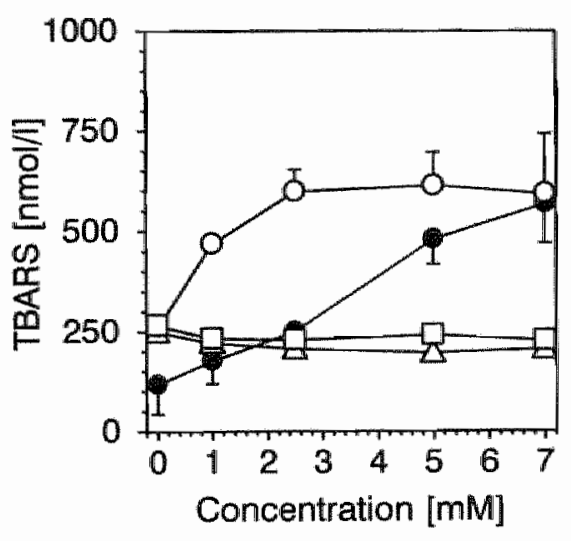

Figure 6.6: Formation of thiobarbituric acid reactive substances in human blood (incubation period 1 hour; $37^{\circ} \mathrm{C}$ ). "The effect of $\square$ CHO, $O A A, \triangle M E K O$ and - HYAM was tested.

Incubation of blood with AAO or HYAM also caused TBARS formation (Figure 6.6). TBARS formation induced by $A A O$ was maximal at $2.5 \mathrm{mM}$ AAO (340 mM; SE=55). TBARS formation induced by HYAM was 78 nM TBARS.mM-1 HYAM and linear over the whole range of concentrations tested $(p<0.0001)$. The effect of AAO on TBARS formation was higher than the effect of HYAM, when test concentrations lower than $2.5 \mathrm{mM}$ were tested. Contradictory to the incubations with hemolysate, AA caused no TBARS formation in blood. No detectable amounts of TBARS were released when blood was incubated with CHO, MEKO, CH and MEK. 
TBARS concentrations were not determined in the co-incubations of erythrocytes and liver samples, as it is not possible to determine whether the effect is cansed by erythrocytes or by the liver sample.

In incubations of hepatocytes with either oximes or HYAM, mo TBARS release was detectable.

\section{Glutathione}

In incubations with hemolysate and AAO, MEKO and HYAM, GT was depleted in a concentration dependent manner $(p<0.01$ in all cases, Figure 6.7). Remarkably, MEKO was the oxime which caused the highest GT depletion $(0.20$ $\mu$ moles per gram hemoglobin for each $\mathrm{mM}$ MEKO added up to a concentration of $5 \mathrm{mM})$. The effect of AAO on $\mathrm{GT}$ was very limited $\left(0.06 \mu\right.$ moles $\cdot \mathrm{g}^{-1} \mathrm{Hb} \cdot \mathrm{mM}^{-1}$ AAO). GT was not affected by the addition of CHO. GT depletion was most pronounced when HYAM was tested $\left(0.47 \mu\right.$ moles. $\mathrm{g}^{-1} \mathrm{Hb} \cdot \mathrm{mM}^{-1}$ HYAM when concentrations up to $5 \mathrm{mM}$ were tested). As with MEKO, GT depletion caused by HYAM did not increase further when concentrations higher than $5 \mathrm{mM}$ were tested.

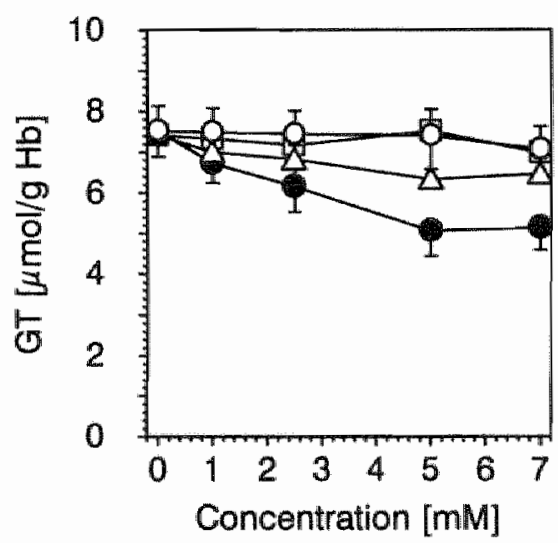

Figure 6.7: Total glutathione concentration in hemolysate (incubation period 1 hour; $37^{\circ} \mathrm{C}$ ). The effect of $\square \mathrm{CHO}, O \mathrm{AAO}, \triangle \mathrm{MEKO}$ and - HYAM was tested.

No effect on GT was found when erythrocytes and oximes were tested. Addition of rat liver microsomes or hepatocytes to these erythrocyte incubations also gave no effect. A large fraction of the initial GT concentration in erythrocytes $\left(6.0 \mu\right.$ moles $\left.\mathrm{g}^{-1} \mathrm{Hb} ; \mathrm{SE}=0.3\right)$ was depleted when HYAM was tested (4.4 $\mu$ moles" $\mathrm{g}^{-1} \mathrm{Hb} ; \mathrm{SE}=0.2$ at $3 \mathrm{mM}$ HYAM). Addition of hepatocytes to these incubations gave about equal results $\left(4.7 \mu\right.$ moles $\left.\cdot \mathrm{g}^{-1} \mathrm{Hb} ; \mathrm{SE}=0.2\right)$. GT concentrations in erythrocytes added to the medium of an isolated perfused rat liver were 
not influenced by the addition of any of the three oximes tested ( $3 \mathrm{mM})$. GT was also not depleted when hepatocytes were incubated with the oximes and HYAM. CH, AA and MEK had no effect on GT in hemolysate, blood or hepatocytes.

\section{Glutathione S-transferase}

All three oximes inhibited GST activity in hemolysate in a concentration dependent manner ( $p<0.001$, Figure 6.8). GST inhibition caused by AAO was not linear and increased from $0.71 \mathrm{U} \cdot \mathrm{g}^{-1} \mathrm{Hb}(\mathrm{SE}=0.2)$ at $1 \mathrm{mM} \mathrm{AAO}$ to 1.04 $\mathrm{U} \cdot \mathrm{g}^{-1} \mathrm{Hb}(\mathrm{SE}=0.2)$ at $7 \mathrm{mM} \mathrm{AAO}$. CHO and MEKO inhibited GST activity in a concentration dependent way $\left(0.27 \mathrm{U} \cdot \mathrm{g}^{-1} \mathrm{Hb}\right.$ for each $\mathrm{mM} \mathrm{CHO}$ added and $0.10 \mathrm{U} \cdot \mathrm{g}^{-1} \mathrm{Hb} \cdot \mathrm{mM}^{-1}$ MEKO resp.). The inhibition of GST activity was most pronounced for HYAM. Like AAO, the greatest effect on GST activity was found at $1 \mathrm{mM}$ HYAM (decrease of $2.38 \mathrm{U} \cdot \mathrm{g}^{-1} \mathrm{Hb} ; \mathrm{SE}=0.2$ ). When $7 \mathrm{mM}$ HYAM was tested, the inhibition of GST activity amounted to $3.12 \mathrm{U} \cdot \mathrm{g}^{-1} \mathrm{Hb}(\mathrm{SE}=0.2)$. No effect on GST activity was found when CH, AA or MEK were tested.

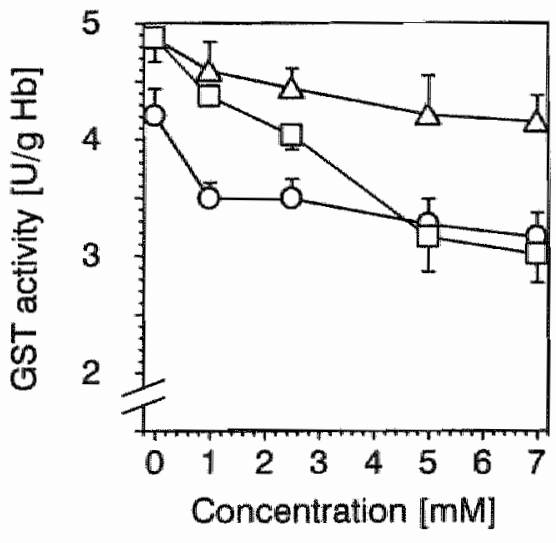

Figure 6.8: Glutathione S-transferase activity in hemolysate (incubation period 1 hour; $37^{\circ} \mathrm{C}$ ). The effect of $\square \mathrm{CHO}$, $O$ AAO and $\triangle$ MEKO was tested.

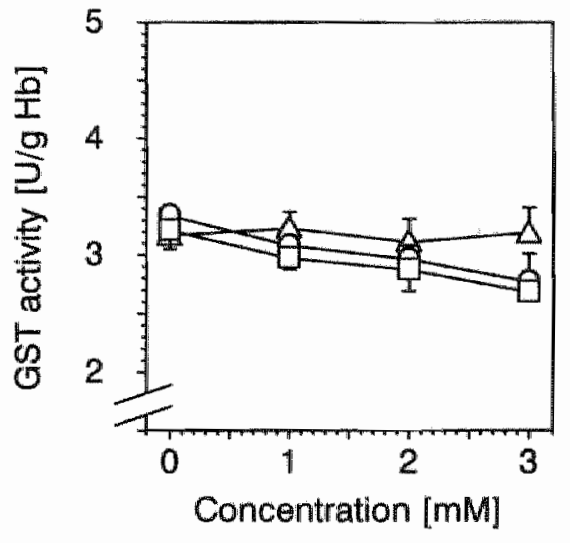

Figure 6.9: Glutathiome Sutransferase activity in erythrocytes (incubation period 1 hour; $37^{\circ} \mathrm{C}$ ). The effect of $\square \mathrm{CHO}$, $O$ AAO and $\triangle$ MEKO was tested.

In incubations with erythrocytes and oximes (Figure 6.9), both CHO and AAO inhibited GST activity in a concentration dependent way $(p<0.001$ in both cases). GST activity decreased with $0.17 \mathrm{U} \cdot \mathrm{g}^{-1} \mathrm{Hb} \cdot \mathrm{mM}^{-1} \mathrm{CHO}$ and with 0.18 $\mathrm{U} \cdot \mathrm{g}^{-1} \mathrm{Hb} \cdot \mathrm{mM}^{-1} \mathrm{AAO}$. MEKO had no influence on GST activity in erythrocytes. When HYAM was tested, GST inhibition amounted to $1.90 \mathrm{U} \cdot \mathrm{g}^{-1} \mathrm{Hb}(\mathrm{SE}=0.9)$ at 3 mM HYAM. No effect on GST activity was found when CH, AA and MEK were tested in blood.

Inhibition of erythrocyte GST activity in co-incubations of erythrocytes and micrasomes and oximes (Figure 6.10) was lower than in incubations with 


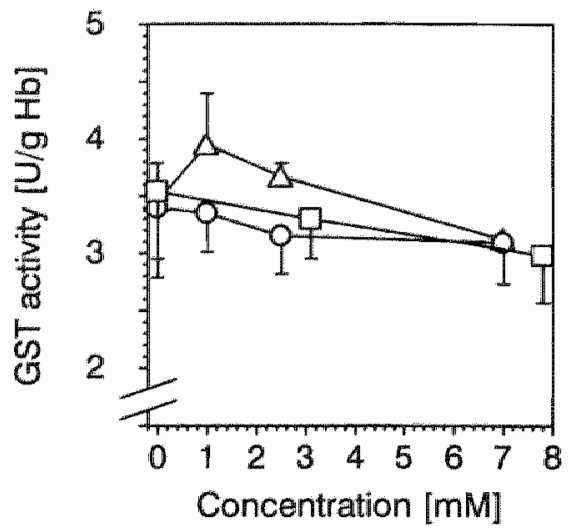

Figure 6.10: Glutathione S-transferase activity in erythrocytes incubated with rat liver microsomes (incubation period 1 hour; $\left.37^{\circ} \mathrm{C}\right)$. The effect of $\square \mathrm{CHO}$, $O A A O$ and $\triangle$ MEKO was tested.

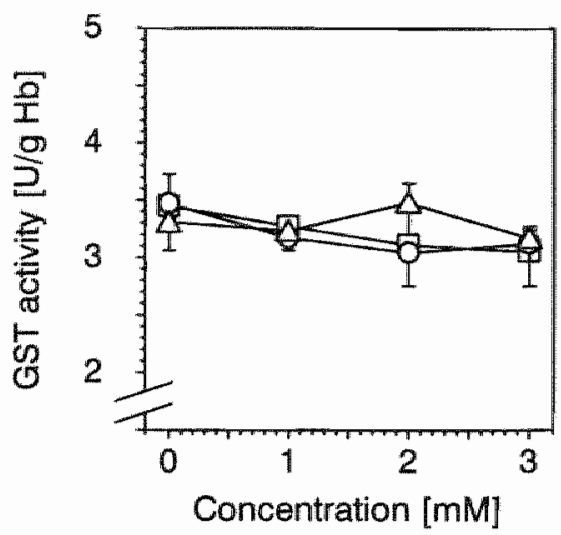

Figure 6.11: Glutathione S-transferase activity in exythrocytes incubated with hepatocytes (incubation period 1 hour; $37^{\circ} \mathrm{C}$. The effect of $\square \mathrm{CHO}, \mathrm{OAAO}$ and $\triangle \mathrm{MEKO}$ was tested.

erythrocytes alone. The GST activity decreased with $0.04 \mathrm{U} \cdot \mathrm{g}^{-1} \mathrm{Hb} \cdot \mathrm{mM}^{-1} \mathrm{AAO}$ $(p=0.0296)$. The effect of CHO and MEKO on GST activity was not statistically significant.

Inhibition of erythrocyte GST activity caused by oximes in co-incubations of erythrocytes and hepatocytes (Figure 6.11) was lower than in incubations with erythrocytes alone. GST inhibition caused by CHO was $0.13 \mathrm{U} \cdot \mathrm{g}^{-1} \mathrm{Hb} \cdot \mathrm{mM}^{-1}$ CHO $(p=0.0819)$. AAO and MEKO had no influence on erythrocyte GST activity.

No time-dependent effect on GST activity in human erythrocytes could be observed when oximes were added to the exythrocyte containing medium of an isolated perfused rat liver. None of the sulstances tested (CHO, AAO, MEKO, HYAM, CH, AA and MEK) had a detectable effect on GST activity in hepatocytes at a concentration of $7 \mathrm{mM}$.

\section{Discussion}

Exposure of rats to the oximes CHO and MEKO causes hemolytic anemia $\llbracket 13$, $21,34]$. It is not well understood whether the hematotoxic effects of these oximes are due to the parent compounds per se or to their metabolites. Parmar and Burka [42] showed that $\mathrm{CHO}$ can be metabolised into $\mathrm{CH}$ and then reduced to cyclohexanol. They suggested that $\mathrm{CH}$ is the active metabolite of $\mathrm{CHO}$ as $\mathrm{CH}$ induces hematotoxic effects. However, HYAM is also released during hydrolysis 
of $\mathrm{CHO}$ and may likewise be responsible for the hematotoxic effects of CHO. In humans many arylhydroxylamines are hemolytic agents that cause methemoglobinemia $[17,30]$. Hydroxylamine and other related compounds not only lead to methemogiobin formation but also produce GT depletion in erythrocytes in vitro $[7,23]$. In addition to the hydrolytic breakdown of oximes, two anaerobic metabolic pathways have been reported; 1) liver aldehyde oxidase metabolizes oximes into ketimines in the presence of the electron donor 2-hydroxypyrimidine. These ketimines will be hydrolyzed nonenzymatically into an oxo-compound and ammonia [50]; no HYAM will be formed in this way 2) acetophenone oxime is reduced by both microsomal and cytosolic enzymes yielding HYAM as one of the products [27]. According to these authors, acetophenone oxime was stable to oxidative and hydrolytic transformations. In contrast, Kohl et al. [32] found that acetoxime is oxdized by human, rat and mice hepatic cytochrome $\mathrm{P}-4501 \mathrm{~A}, 2 \mathrm{~B}$ and $2 \mathrm{E}_{1}$ into propane 2-nitronate, which is a pottent genotoxicant and carcinogen, and than reduced into 2-nitropropane. Both acetoxime and 2nitropropane cause elevated levels of 7 -hydroxyguanine in liver DNA and RNA in rat $[10,11,19,20,25]$.

In this study we tested on one hand whether the oximes CHO, AAO and MEKO as such are able to generate oxidative effects in human erythrocytes, and on the other hand whether the suggested metabolites of these oximes, CH, AA, MEK, respectively and HYAM are hematotoxic. Oximes were also tested in a combined assay of human erythrocytes with microsomes or hepatocytes, and in an isolated rat liver perfusion in which human erythrocytes were added to the medium to investigate whether possible metabolites generated in these test systems are able to generate oxidative effects in erythrocytes and/or hepatocytes.

$\mathrm{HbFe}^{3+}$ accumulation, TBARS formation, GT depletion and GST activity changes were tested. We chose these parameters since a) in vivo exposure to CHO causes hemoglobin oxidation and Heinz body formation $[13,21]$ b) we previously found that HYAM, a possible metabolite of oximes, affects all four parameters [16]. During the process of $\mathrm{HbFe}^{3+}$ formation, free radicals are formed [49]. These free radicals may be the initiators for the TBARS formation that occurred. They can also give rise to the generation of protein disulfides, mixed disulfides or oxidized glutathione [24]. Lipid peroxides can be reduced into alcohols by glutathione peroxidase or glutathione S-transferase [18], and protein disulfides or mixed disulfides can be reduced by thioltransferase, with concomitant oxidation of GSH [36]. These processes may lead to a reduction of erythrocyte GSH levels $[18,33]$. Erythrocyte GST, which is a $\pi$-class enzyme, is known to be vulnerable to oxidative stress $[14,16,45,46]$ and electrophiles $[6,35]$, and was therefore also included as an effect parameter in the experiments.

The results show that the oximes and FYAM all caused hemoglobin oxidation in erythrocytes. Although the $\mathrm{HbFe}^{3+}$ accumulation for HYAM was not higher in 
hemolysate than in erythrocytes, this was clearly the case for the oximes. This indicates that uptake of the oximes by the erythrocytes may be a limiting factor. The magnitude of hemoglobin oxidation by oximes in erythrocytes was low compared to HYAM and lowest for MEKO. This was also the case in hemolysates. Therefore, although uptake limitation cannot be ruled out, it is not the only factor determining potency differences between oximes and HYAM. Incubation of the possible oxime metabolites $\mathrm{CH}, \mathrm{AA}$ or $\mathrm{MEK}$ caused no $\mathrm{HbFe}^{3+}$ accumulation, showing that, even if $\mathrm{CH}, \mathrm{AA}$ and MEK are metabolites of the oximes, they are not responsible for methemoglobin formation. Co-incubation of human erythrocytes and rat liver preparations (i.e. microsomes, hepatocytes, whole liver) did not affect $\mathrm{HbFe}^{3+}$ accumulation by oximes. However, possible metabolites generated by hepatocytes may not be able to reach their target in the erythrocyte. No effects on hepatocyte GT depletion, TBARS formation and GST activity were found in incubations of hepatocytes with CHO, AAO or MEKO. All in all, this indicates that the formation of reactive oxime metabolites by hepatocytes is of minor importance for oxime activation. A comparison between the in vitro and in vivo results [13] concerning $\mathrm{HbFe}^{3+}$ accumulation is difficult. Although the in vivo intravascular CHO concentration is not known, it was probably in the millimolar range. Also, the longer duration of the in vivo experiments may have lead to cumulative effects. Sequestration of erythrocytes containing high amounts of $\mathrm{HbFe}^{3+}$ accumulated in the spleen may have obscured these effects. Furthermore, a difference in vulnerability may exist between human and rat erythrocytes.

Lipid peroxidation was measured as TBARS production. TBARS production in human blood was found only for AAO and HYAM. Opposed to what was found for HYAM, the TBARS formation caused by AAO was much higher in hemolysate than in blood. Uptake limitation for AAO in the erythrocyte may be the reason for this. AA, a possible metabolite of AAO only caused a detectable TBARS formation in hemolysate. AA is a substrate for xanthine oxidase, and the generation of free radicals in this reaction [39], may explain the TBARS formation in hemolysate. However, additional experiments with the xanthine oxidase inhibitor allopurinol showed that the role of xanthine oxidase activity in the induction of TBARS formation by AA in hemolysate is negligible (data not shown). The magnitude of the TBARS formation caused by AAO found in hemolysate was about the sum of the effects found for AA and HYAM. Therefore, hydrolysis of AAO to AA and HYAM could explain the TBARS formation inducing activity of AAO. In hepatocytes, no TBARS were formed by oximes or HYAM. This together with the known generation of free radicals during the $\mathrm{HbFe}^{3+}$ formation by HYAM [49] leads to the conclusion that HYAM needs hemoglobin to induce TBARS formation. This was confirned by the observation that no TBARS were formed when HYAM was incubated with washed red blood cell membranes (data not shown). Considering $A A O$, a factor from the erythrocytes also seems to be required fon: TBARS formation as AAO caused no TBARS in hepatocytes. 
Glutathione (GT) in erythrocytes was depleted only by HYAM. In hemolysate. AAO and MEKO as well as HYAM caused GT depletion. However, the magnitude of the effect caused by AAO was small. Comparing the magnitude of the effects caused by HYAM on erythrocyte HbFe ${ }^{3+}$ accumulation, TBARS and GST, erythrocyte GT depletion was the least sensitive parameter. As the effects of oximes on $\mathrm{HbFe}^{3+}$ accumulation, TBARS and GST were much lower than the effects of HYAM, we expected no effect of the oximes on erythrocyte GT. Interestingly, MEKO, which was the only oxime that did not cause GST inhibition in erythrocytes, did give rise to GT depletion. The most probable explanation for this GT depletion is glutathione conjugation of MEKO or MEKO metabolites that may be formed by the cytosol of erythrocytes. No GT depletion was found when hepatocytes or microsomes were added to the incubations with erythrocytes, which is in accordance with the fact that $\mathrm{HbFe}^{3+}$ accumulation was generally not influenced by the addition of liver preparations. Hepatic glutathione was also not affected by oximes or HYAM. As hepatocytes contain about $10 \mathrm{mM}$ glutathione [9] and erythrocytes only about $2 \mathrm{mM}$, the higher glutathione concentration in hepatocytes may mask GT depletion. The fact that GT depletion caused by oximes was not higher in erythrocytes after the addition of liver preparations confirms that no important formation of erythrotoxic reactive metabolites occurs under these experimental conditions.

Glutathione S-transferase (GST) activity decreased when erythrocytes were incubated with CHO, AAO or HYAM. The effect was more pronounced when hemolysate was tested, and in this case even MEKO induced a decrease in GST activity. GST- $\pi$ (comparable to erythrocyte GST- $\rho$ ) is known to be vulnerable for free radicals and lipid peroxidation products $[14,16,45,46]$. The strong erythrocyte GST inhibition caused by HYAM or AAO (both compounds induced TBARS formation) can be a consequence of free radicals or toxic products generated during TBARS formation. The small GST inhibitory capacity of MEKO in incubations with hemolysate may be a consequence of marginal TBARS formation. CHO and AAO inhibited erythrocyte GST to the same extent. However, CHO caused no TBARS formation in erythrocytes. For this reason we expected to find a GST inhibition which was comparable to the GST inlhibition found for MEKO. As the erythrocyte GST inhibition caused by CHO was much higher, inhibition of GST works via a different mechanism from that found for AAO and HYAM. Many compounds are known to inhibit GST activity directly by modification of the enzyme [35]. CHO may function in the same way. All effects on GST were lower when microsomes or hepatocytes were added to the incubations with erythrocytes. This could be a result of impaired availability of the test compound caused by absorption by the hepatocytes or microsomes (e.g. protein binding).

Based on our in vitro results, we conclude that a) oximes have less oxidative capacity than HYAM. b) AAO may be regarded as more erythrotoxic than CHO and MEKO, as it causes TBARS formation. c) hemoglobin oxidation, TBARS 
formation and GST activity decrease are generally related to each other. However, specific GST inhibition (e.g. CHO) disturbs this general relationship. d) compared to $\mathrm{HbFe}^{3+}$ accumulation and GST inhibition, GT depletion is a less sensitive parameter for oxidative compounds and may be strongly infuenced by compounds metabolised by GSH conjugation, as is possibly the case for MEKO e) the oxo-compounds related to the oximes tested gave no effect with the exception of AA in TBARS formation $f$ ) the inclusion of liver preparations had no effect on erythrocyte parameters. This indicates that liver metabolism is not of major importance for the hematotoxic effects of oximes.

Acknowledgements The authors wish to thank M. Leummens, L. Baars and R. Bisschops for their technical support, Dr. J. Neis of DSM for providing the oximes and for critical reading of the manuscript, Dr. A. Kester for his advice in statistical analyses, and the Red Cross Blood Bank "Zuid Limburg" for collecting the blood samples. 


\section{Bibliography}

11] Anderson, M.E. (1985) Determination of glutathione and glutathione disulfide in biological samples. Methods Enzymol. 113, 548-555.

[2] Bast, A. and Goris, R.J.A. (1989) Oxidative stress; biochemistry and human disease. Pharm. Weekbl. Sci. 11, 199-206.

[3] Berry, M.N. and Friend, D.S. (1969) High yield preparation of isolated rat liver parenchymal cells. J. Cell Biol. $43,506-520$.

[4] Betke, K., Stein, H. and Tön, O. Determination of methemoglobin and sulfhemoglobin, in: Tietz N.W. (ed.) Textbook of clinical chemistry, W.B. Saunders Company, Philadelphia, 1986, pp. 1495-1588.

[5] Board, P., Coggan, M., Johnstion, P., Ross, V., Suzuki, T. and Webb, G. (1990) Genetic heterogeneity of the human glutathione transferases: a complex of gene families. Pharm. Therap. 48, 357-369.

[6] Brouwer, E.J., Evelo, C.T.A., Vexplanke, A.J.W., wan Welie, R.T.H. and de Wolff, F.A. (1991) Biological effect monitoring of occupational exposure to 1 , 3 . dichloropropene: effects on liver and renal function and on glutathione conjugation. Br. J. Ind. Med. 48, 167-172.

[7] Calabrese, E.J., Tilli, F. Horton, H.M. and Stoddard, A. (1988) The effects of ethanol on the hematotoxicity of twelve pharmacentical and environmental agents. J. Environ. Sci. Health A23, 359-367.

[8] Carrell, R.W., Winterbourn C.C. and Rachmilewitz E.A. (1975). Activted oxygen and haemolysis. Br. J. Haematol. 30, 259-264.

[9] Coles, B. and Ketterer, B. (1990) The role of glutathione and glutathione transferases in chemical carcinogenesis. Crit. Rev. Biochem. Mol. Biol. 25, 47-66.

[10] Conaway, C.C., Nie, G., Hussain, N.S., Asaad, W.W. and Fiala, E.S. (1990) Secondary nitroalkanes and ketoximes induce oxidative damage to DNA and RNA. Carcinogenesis $31,145$.

[11] Conaway, C.C., Nie, G.N., Hussain, N.S. and Fiala, E.S. (1991) Comparison of oxidative damage to rat liver DNA and RNA by primary nitroallanes, secondary nitroalkanes, cyclopentanone oxime, and related compounds. Cancer Res. 51, 31433147 .

[12] Deneke, S.M. and Fanburg. B.L. (1989) Regulation of cellular glutathione. Am. J. Plyysiol. 257, L163-L173.

[13] Derelanko, M.I., Gad, S.C., Powers, W.J., Mulder, S., Gawigan, F, and Babich, P.C. (1985) Toxicity of cyclohexanone mime; I. Hematotoxicity following subacute exposure in rats. Fundam. Appl, Toxicol. 5, 117-127.

[14] Evelo, C.T.A., Pamen, N.G.M., Arthur, Y. and Janssen, G.M.E. (1992) Changes in blood glutathione concentrations, and in erythrocyte glutathione reductase and glutathione S-transferase activity after running training and after participation in contests. Eur. J. Appl. Physiol. 64, 354-358.

[15] Evelo, C.T.A., Bos, R.P. and Borm, P.J.A. (1993) Decreased glutathione content and glutathione S-transferase antivity in red blood cells of coal miners with early stages of prieumoconiosis. Br. J. Ind. Med. 50, 683-636. 
[16] Evelo, C.T.A. Toxicological stress indicators in human red blood cells; changes in glutathione and glutathione $\mathbf{S}$-transferase as biological markers for electrophilic and oxidative stress, University Press, PhD Thesis, Maastricht, 1995, pp. 77-100.

[17] Eyer, P. (1983) The red cell as a sensitive target for activated toxic arylamines. Arch. Toxicol. Suppl. 6, 3-12.

[18] Fabey, R.C. and Sundquist, A.R. Erolution of glutathione metabolism, in: Meister, A. (ed.) Adv. Enzymol. Relat. Areas Mol. Biol., J. Wiley and Sons, New York, Vol 64, 1991, pp. 1-53.

[19] Fiala, E.S., Conaway, C.C., Biles, W.T. and Johnson, B. (1987) Enhanced mutagenicity of 2-nitropropane nitronate with respect to 2-nitropropane; possible involvement of free radical species. Mutat. Res. $179,15-22$.

[20] Fiala, E.S., Conaway, C.C. and Mathis, J.E. (1989) Oxidative DNA and RNA Damage in the livers of Sprague-Dawley rats treated with the hepatocarcinogen 2-nitropropane. Cancer Res. 49, 5518-5522.

[21] Gad, S.C., Derelanko, M.J., Powers, W.J., Mulder, S., Gavigan, F. and Babich, P.C. (1985) Toxicity of cyclohexanone oxime: II. Acute dermal and subchronic oral studies. Fundam. Appl. Toxicol. 5, 128-136.

[22] Gordon-Smith, E.C. (1980) Drug induced oxidative havemolysis. Clin. Haematol. $9,557-586$

[23] Gross, P. (1985) Biologic activity of hydroxylamine: A review. Crit. Rev. Toxicol. $1.4,87 \sim 99$.

[24] Grossman, S.J., Simson, J., and Jollow, D.J. (1992) Dapsone-induced hemolytic anemia: effect of $\mathbb{N}$-hydroxy dapsone on the sulfhydry $\sharp$ status and membrane proteins of rat erythrocytes. Toxicol. Appl. Pharmacol. 117, 208.

[25] Guo, N., Conaway, C.C., Hussain, N.S. and Fiala, E.S. (1990) Sex and organ differences in oxidatieve DNA and RNA damage due to treatment of SpragueDawley rats with acetoxime or 2-nitropropane. Carcinogenesis 11, 1659-1662.

[26] Habig, W.H. and Jakoby, W.B. (1981) Assay for differentiation of glutathione S-transferases. Methods Enzymol. 77, 398-405.

[27] Hes, J and Sternson, L.A. (1974) The metabolism of acetophenone oxime in rat: liver homogenates. Drug Metab. Dispos. 2, 345-350.

[28] Hucker, H.B., Michniewicz, B.M., and Rhodes, R.E. (1971) Phenylacetone oxime; an intermediate in the oxidative deamination of amphetamine. Biochem. Pharmacol. $20,21123-2128$.

[29] Jauregui, H.O., Hayner, N.T, Driscoll, J.L., Williams-Holland, R., Lipski, M.H. and Galletti P.M. (1981) Trypan blue dye uptake and lactate dehydrogenase in adult rat hepatocytes-freshly isolated cells, cell suspensions, and primary monolayer cultures. In Vitro Cell. Dev. Biol. 17, 1100-1110.

[30] Jensen, C.B. and Jollow, D.J. (1991) The role of N-hydroxyphenetidine in phenacetin induced hemolytic anemia. Toxicol. Appl. Pharmacol. 111, 1-12.

[31] Kampen van, E.J. and Zijlstra, W.G. (1965) Determination of hemoglobin and its derivates. Adv. Olin. Chem. 8, 141-187.

[32] Kolal, C., Schiller, C.D., Gescher, A., Farmer, P.B. and Bailey, E. (1992) Acetoxime is metabolized by human and rodent hepatic cytochrome P-450 enzymes to the genotoxicant and carcinogen propane 2-nitronate. Carcinogenesis 13, 1091-1094. 
[33] Kondo, T., Dale, G.L. and Beutler, E. (1981) Studies on glutathione transport utilizing inside-out vesicles prepared form human erythrocytes. Biochin. Biophys. Acta $645,132-136$.

[34] Kurita, H. (1967) Experimental studies on methyl-ethyl-ket-oxime toxicity. Nagoya J. Med. Sci. 29, 393-418.

[35] Mannervik, B. and Danielson, U.H. (1988) Glutathione transferases; stucture and. catalytic activity. C.R.C. Crit. Rev. Biochem. 23, 283-337.

[36] Mieyal, J.J., Starke, D.W., Gravina, S.A. and Hocevar, B.A. (1991) Thioltransferase in human red blood cells: kinetics and equilibrium. Biochemistry $30,8883-$ 8891.

[37] Moss, D.W., Henderson, A.R. and Kachmar, J.F. Enzymes. in: Tietz, N.W. (ed.) Textbook of clinical chemistry, W.B. Saunders Company, Philadelphia, 1986, pp. 619-762.

[38] Murata, T., Hatayama, I., Satoh, K., Tsuchida, S. and Sato, K. (1990) Activation of rat glutathione transferases in class mu by active oxygen species. Biochem. Biophys. Res. Commun. 171, 845-851.

[39] Nordback, I.H., MacGowan, S., Potter, J.J. and Cameron, J.L. (1991) The role of acetaldehyde in the pathogenesis of acute alcoholic pancreatitis. Anr. Surg. 214, $671-678$.

[40] Palmen, N.G.M., Evelo, C.T.A., Borm, P.J.A. and Henderson, P.Th. (1992) Influence of oxygen supply on liver condition and elimination of dimethylacetamide in the isolated perfused rat liver. Toxicol. in Vitro 6, 357-365.

[41] Palmen, N.G.M. and Evelo, C.T.A. (1993) Glutathione depletion in human erythrocytes as an indicator for microsomal activation of cyclophosphamide and 3hydroxyacetanilide. Toxicology 84, 157-170.

[42] Parmar, D. and Burka, L.T. (1991) Metabolism and disposition of cyclohexanone oxime in male F-344 rats. Drug Metab. Dispos. 19, 1101-1107.

[43] Rutten, A.A.J.J.L., Falle, H.E., Catsburg, J.F., Topp, R., Blaauboer, B.J., van Holsteijn, I., Doorn, L. and Rolaf van Leewwen, F.X. (1987) Interlaboratory comparison of total cytochrome P.450 and protein determinations in rat liver microsomes; Reinvestigation of assay conditions. Arch. Toxicol. 61. 27-33.

[44] Seglen, P.O. (1973) Preparation of rat liver cells. Exp. Cell Res. 82, 391-398.

[45] Shen, H., Tamai, K., Satoh, K., Hatayama, I., Tsuchida S. and Sato, K. (1991) Modulation of class pi glutathione transferase activity by sulfhydryl group modification. Arch. Biochem. Biophys. 286, 178-182.

[46] Shen, H., Tsuchida, S. Tamai, K. and Sato, K. (1993) Identification of cysteine residues inwolved in disulfide formation in the inactivation of glutathione transferase P-form by hydrogen peroxide. Arch. Biochem. Biophys. 300, 137-141.

[47] Sinnhuber, R.O. and Yu, T.C. (1957) 2-Thiobarbituric acid method for the mea surement of rancidity in fishery products; II The quantitative determination of malonaldehyde. Food Technol. $12,9-12$.

[48] Stocks, J. and Dormandy, T.L. (1971) The autoxidation of human red cell lipids induced by hydrogen peroxide. Br. J. Haematoll 20, 95-111. 
[49] Stolze, K and Nohl, H. (1989) Detection of free radicals as intermediates in the methemoglobin formation from oxyhemoglobin induced by hydroxylamine. Biochem. Pharmacol. 38, 3055-3059.

[50] Tatsumi, K. and Ishigai, M. (1987) Oxime-metabolizing activity of liver aldehyde oxidase. Arch. Biochem. Biophys. 253, 413-418.

[51] Weed, R.I. (1970) The importance of erythrocyte deformability. Am. J. Med. 49, $147-150$. 


\section{Chapter 7}

\section{General discussion}

Blood is the fluid circulating through the body carrying mutrients and oxygen through erythrocytes and plasma to body cells and removing waste products and carbon dioxide. During its circulation through the arteries and veins, erythrocytes come in close contact with organs. One of these organs is the liver. The liver has the highest metabolic capacity and as metabolites generated by the hepatocytes may leave these cells, erythrocytes can be exposed to products of biotransformation. As a result of this exposure, targets or biochemical reactions in erythrocytes may be affected.

In this thesis, the interplay between erythrocytes and hepatocytes was studied with special attention to bio-activation and inactivation capacity of both liver and blood. In order to gain an insight in this problem, several in vitro techniques had to be considered and developed.

The primary reasons to use human blood cells were (i) to facilitate extrapolation from the in vitro to the human in vivo situation and (ii) that blood may be applicable in biological effect monitoring of workers exposed to chemicals. Moreover, the erythrocyte is a relatively vulnerable blood cell since it possesses no mucleus and organelles [11], so it is totally dependent on glycolysis for its need of ATP [28]. The absence of a nucleus and endoplasmic reticulum makes that erythrocytes cannot synthetize proteins. This means that loss of enzyme activity its irreversible since it cannot be compensated by the synthesis of new enzyme. The transport of oxygen, which is the most important function of erythrocytes, is dependent on ferrous hemoglobin. Oxidized hemoglobin can be reduced by means of NADH cytochrome $b_{s}$ reductase and NADPH flavin reductase using NADH and NADPH tespectively, as reducing equivalents $[21,31,32]$. Moreover, the reductant NADPH is needed in several other biochemical reactions (e.g. reduction of oxidized glutathione $[15,17])$. The need for NADPH in the erythrocyte is totally fulfilled by the hexose monophosphate shunt.

The effect parameter which was studied most intensely in this thesis the is glutathione content. Glutathione is a tripeptide present both in erythrocytes and hepatocytes. Cellular glutathione stores can be depleted by means of conjugation 
of reduced glutathione (GSH) to electrophilic substrates, the generation of mixed disulfides [10] or by export of oxidized glutathione (GSSG) from the cell [1, 13]. Several target systems containing GSH as a molecular target were used (Fig-

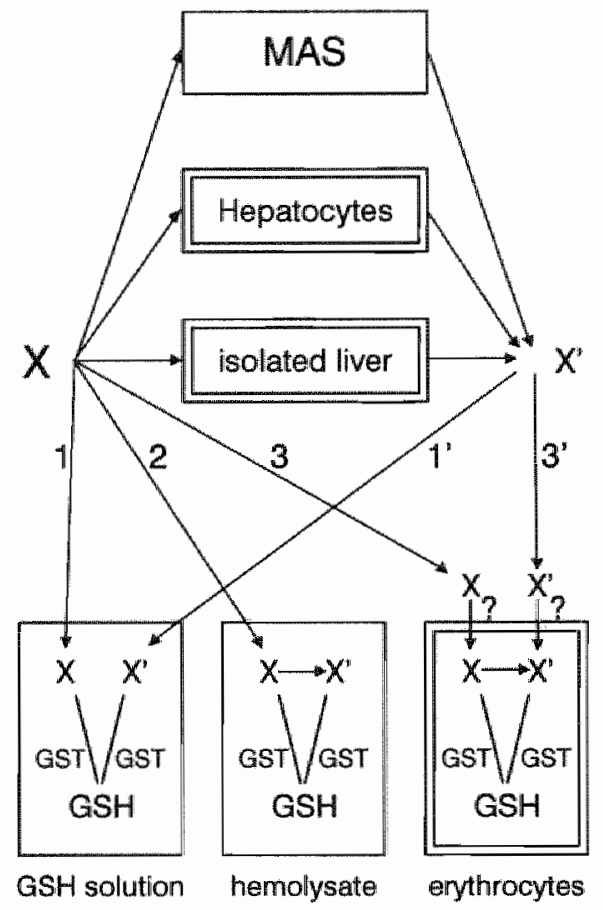

Figure 7.1: An outline of the incubations performed in this thesis. The picture is explained in the text. 1) glutathione solution 2) hemolysate 3) erythrocytes 1') glutathione solution plus microsomal activating system (MA.S) or hepatocytes 3 ') erythrocytes plus MAS, hepatocytes or whole liver

ure 7.1). The results obtained in this thesis using different GSH sources and substrates are summarized in Table 7.1.

The ability of several compounds with different mechanisms of action to react with GSH was tested in incubations with GSH solution (arrow 1). We found that iodoacetamide, N-ethylmaleimide and diethyl maleate strongly depleted the amount of GSH in solution (see also Table 7.1). These compounds were also able to deplete erythrocyte GSH (arrow 3) which means that they can pass the erythrocyte membrane. Another group of compounds is not able to deplete total glutathione (GT, which is the sum of both GSH and GSSG) in incubations with GSH solution. Cyclophosphamide, 3-hydroxyacetanilide, 2-methylfurane, monuron and monulinuron belong to this group. Erythrocytes are metabolically 
Table 7.1: Qualitative summary of the test results on glutathione depletion, obtained with the different test systems and substrates used in this thesis.

\begin{tabular}{|c|c|c|c|c|c|c|c|}
\hline \multirow{2}{*}{$\begin{array}{c}\text { Substrate } \\
+ \text { (Chapter) }\end{array}$} & \multicolumn{2}{|c|}{ GSH solution } & \multirow{2}{*}{$\begin{array}{l}\text { Hemo- } \\
\text { lysate }\end{array}$} & \multicolumn{2}{|c|}{ Erythrocytes } & \multirow{2}{*}{$\begin{aligned} & \text { Erys } \\
+ & \text { Hep }\end{aligned}$} & \multirow{2}{*}{$\begin{array}{c}\text { Perfu- } \\
\text { sion }\end{array}$} \\
\hline & - MAS & $+\mathrm{MAS}$ & & -MAS & +MAS & & \\
\hline $\operatorname{IAcA}(3)$ & + & & & + & & & \\
\hline NEM (3) & + & & & + & & & \\
\hline DEM (3) & + & & & + & & & \\
\hline HYAM $(6)$ & & & + & + & & + & \\
\hline $\operatorname{CP}(2,3)$ & 0 & + & 0 & 0 & + & & \\
\hline $3-\mathrm{HAA}(2,3)$ & 0 & + & + & 0 & + & 0 & \\
\hline $2-\operatorname{MF}(3)$ & 0 & + & + & 0 & + & & \\
\hline$M N(3)$ & 0 & 0 & 0 & 0 & 0 & & \\
\hline $\operatorname{MLN}(3)$ & 0 & 0 & 0 & 0 & 0 & & \\
\hline $\mathrm{CHO}(6)$ & & & 0 & 0 & 0 & 0 & 0 \\
\hline $\mathrm{AAO}(6)$ & & & $+/ 0$ & 0 & 0 & 0 & 0 \\
\hline MEKO (6) & & & + & 0 & 0 & 0 & 0 \\
\hline
\end{tabular}

Glutathione depletion was measured in incubations with reduced glutathione (GSH), hemolysate or erythrocytes. The effect of addition of (i) microsomal activating system of normal rats (MAS) to incubations with GSH solution or erythrocytes (ii) hepatocytes to erythrocytes (Erys+Hep) or (iii) a whole liver perfused with erythrocytes (perfusion) was also tested. Depletion of glutathione by a compound is indicated with $(+)$, a marginal effect is indicated as $(+/ 0)$ and no effect on glutathione as $(0)$. Testsubstrates were (IACA) iodoacetamide, (NEM) N-ethylmaleimide, (DEM) diethyl maleate, (CP) cyclophosphamide, (HYAM) hydroxylamine, (3-HAA) 3-hydroxyacetanilide, (2-MF) 2-methylfurane, (MN) monuron, (MLN) monulinurom, (CHO) cyclohexanone oxime, (AAO) acetaldoxime and (MEKO) methyl-ethyl ketoxime.

Significance of glutathione depletion was determined by linear regression of the slope of GT depletion versus concentration of the substrate. The substrate concentrations tested differed between compounds and are described in the respective chapters. The samples were incubated for 1 hour at $37^{\circ} \mathrm{C}$. Depletion of reduced glutathione was measured when IACA, NEM and DEM were tested. Total glutathione depletion was measured for the other compounds.

active $[3,5,16,19,20,29]$. If compounds belonging to the group described above are also metabolically activated in the erythrocytes, they may afterwards be able to deplete GT in these incubations. However, no GT depletion in erythrocytes was found when cyclophosphamide, 3-hydroxyacetanilide, 2 methylfurane, monuron and monulinuron were tested. As the amount of compound that passed 
the erythrocyte membrane during the 1 hour incubation period may be too low because of diffusion limitation, hemolysates were also incubated with the nonreactive compounds. Both 3-hydroxyacetanilide and 2-methylfurane depleted the GT content in hemolysates. This may indicate that GSH-reactive metabolites are formed by the hemolysates. However, the absence of a cellular membrane may not be the only difference between erythrocytes and hemolysate. Cyclophosphamide did not affect the GT concentration in hemolysates. The strong oxidative compound hydroxylamine was able to deplete the GT concentration in both incubations with erythrocytes and hemolysate. Since oximes might be metabolised by erythrocytes into hydroxylamine and the respective oxomcompound, GT reactive metabolites might be formed. However, no GT depletion was detected in incubations with erythrocytes and cyclohexanone oxime, acetaldoxime and methyl-ethyl ketoxime. However, methyl-ethyl ketoxime and, to a marginal extent, acetaldoxime were able to deplete GT in incubations with hemolysate. The difference in results obtained with erythrocytes and hemolysates may not only be due to the membrane but also to for instance differences in reductive capacity due to higher oxygen exposure of the hemolysates.

To study the interplay between hepatic and erythrocyte metabolism several in vitro techniques were used in which rat liver derived tissue was added to incubations with GSH solution or erythrocytes (Figure 7.1, arrow 1' and 3'). Rat liver was used because it is very difficult to obtain human livers. A disadvantage of the use of rat tissue is that the biotransformation pathways between rat and man may be different, so that the results obtained in the incubations using rat liver derived tissues may not apply to the human situation. The test compounds which did not deplete GT in the incubations with GSH solution and erythrocytes were tested again in these systems but now in the presence of rat liver microsomal activating system (MAS). Cyclophosphamide, 3-bydroxyacetanilide and 2-methylfurane were metabolised by MAS into GSH-reactive compounds as GT was depleted both in incubations with GSH solution and erythrocytes (Table 7.1). Implicitly this means that the metabolites generated by MAS were able to pass the erythrocyte membrane. The generation of the GSH-reactive metabolites was NADPH dependent which is indicative of activity of the cytochrome P-450 complex. GT depletions were increased when microsomes derived from rats pretreated with phenobarbitone were added. This indicates that the phenobarbitone inducable enzymes, which include some isoenzymes of cytochrome P-450, also participate in the generation of the GSH-reactive cyclophosphamide, 3-hydroxyacetanilide and 2-methylfurane metabolites. GT was not depleted when the pesticides monuron and monulinuron were tested in incubations with GSH solution or erythrocytes with or without the addition of MAS. The three oximes were also not metabolised by MAS into detectable amounts of GSH-reactive metabolites. Although there is no definitive explanation for this phenomenon, the following options may be the case (i) no oxime metabolites are formed by the liver (ii) possible metabolites 
formed in the test system are not able to pass the erythrocyte membrane or (iii) do not conjugate with GSH.

A test system more closely imitating the physiological situation than erythrocytes and MAS, consisted of co-incubated human erythrocytes and rat hepatocytes (Figure 7.1, arrow 3"). In contrast to the previous systems, GSH-reactive metabolites in this model must first cross the hepatocyte and Transwell ${ }^{\text {TM }}$ membrane to reach erythrocyte targets. Rat hepatocytes are a metabolically very active cell type but they also have a high detoxification capacity [8]. This means that GSHreactive metabolites may be formed by hepatocytes but also that they may be detoxified within the hepatocyte. As a result of this intracellular detoxification, less GSH-reactive metabolites will leave the hepatocyte. Cyclophosphamide and 3-hydroxyacetanilide were metabolised by hepatocytes into GSH-reactive compounds since they depleted the amount of intracellular GT (Chapter 3). Interestingly, the GSH-reactive metabolites of both cyclophosphamide and, notably, 3-hydroxyacetanilide generated by the hepatocytes were also excreted into extracellular milieu (Chapter 3). For this reason, 3-hydroxyacetanilide was tested in co-incubations of hepatocytes and erythrocytes. Metabolites generated by the hepatocytes were expected to pass the hepatocyte and the erythrocyte membrane to deplete erythrocyte GT. However, no effect on erythrocyte GT could be detected, which may be explained by uptake limitation of the GSH-reactive metabolite(s) into the erythrocyte (Table 7.1). Also no GT depletion was found when cyclohexanone oxime, acetaldoxime and methyl-ethy! ketoxime were tested in co-incubations of hepatocytes and erythrocytes. Oximes may be metabolised by hepatocytes into hydroxylamine, a strongly oxidative compound, able to pass the hepatocyte and the erythrocyte membrane and oxidize erythrocyte GSH in previous experiments. Since neither erythrocyte nor hepatocyte GT were affected by the oximes this indicates that no GSH-reactive oxime metabolites are formed in the hepatocytes.

The in vitro technique closest to the in vivo situation was the isolated perfused liver. Since no effects on erythrocyte GT were found in co-incubations of erym throcytes and hepatocytes testing 3-bydroxyacetanilide or the oximes, it was not expected that erythrocyte GT would be affected in the liver perfusions. This was confirmed in liver perfusion experiments in which the oximes were tested (Table 7.1).

Oximes are known oxidative compounds in vivo that may be metabolised in the body into oxo-compounds and hydroxylamine. In addition to GT depletion, hydroxylamine can oxidize hemoglobin, cause lipid peroxidation and affect erythrocyte GST activity [7]. Therefore, these parameters were also assessed in our studies on the effects of oximes on human erythrocytes. In fact, a detailed comparison of several effect markers showed that depletion of GT is a relatively insensitive parameter to evaluate oxidative effects in blood (Table 7.2). $\mathrm{HbFe}^{3+}$ 
Table 7.2: Hemoglobin oxidation, lipid peroxidation, glutathione depletion and inhibition of glutathione S-transferase activity in hemolysate and erythrocytes caused by oximes and hydroxylamine.

\begin{tabular}{|l|cccc|cccc|}
\hline & \multicolumn{4}{|c|}{ hemolysate } & \multicolumn{4}{c|}{ erythracytes or blood } \\
\cline { 2 - 9 } Substrate & HbFe $^{3+}$ & TBARS & GT & GST & HbFe & TBARS & GT & GST \\
\hline HYAM & + & + & + & + & + & + & + & + \\
CHO & + & 0 & 0 & + & + & 0 & 0 & + \\
AAO & + & + & $+/ 0$ & + & + & + & 0 & + \\
MEKO & + & 0 & + & + & + & 0 & 0 & 0 \\
\hline
\end{tabular}

(HbF $\mathrm{e}^{3+}$ ) hemoglobin oxidation, (TBARS) formation of thiobarbituric reactive substances, (GT) total glutathione depletion and (GST) glutathione S-transferase activity clanges caused by different substrates were tested in incubations with hemolysate and erythrocytes or blood. When the parameter tested is affected by the test compound this is indicated by $(+)$. No effect of the test compound or a marginal effect are indicated by (0) and ( $+/ 0)$ respectively. Testsubstrates were (HYAM) hydroxylamine, (CHO) cyclohexanone oxime, (AAO) acetaldoxime and (MEKO) methyl-ethyl ketoxime. In contrast to the other parameters in which washed erythrocytes were used, formation of TBARS was tested in whole blood. Samples were incubated for 1 hour at $37^{\circ} \mathrm{C}$.

Statistical significance of the effect found was determined by linear regression of the slope of the substrate concentration versus the parameter measured. Substrate concentrations up to $7 \mathrm{mM}$ were tested in incubations with hemolysate. In incubations with erythrocytes or blood, substrate concentrations up to $3 \mathrm{mM}$ were tested except for TBARS formation in blood which was tested up to $7 \mathrm{mM}$.

formation and GST inhibition were much better parameters as they were generally affected by the oximes while GT was only markedly depleted by methyl-ethyl ketoxime in hemolysate and hydroxylamine in both erythrocytes and hemolysate. Although $\mathrm{HbFe}^{3+}$ formation was most pronounced when oximes were incubated with hemolysate, also in erythrocytes $\mathrm{HbFe}^{3+}$ formation was observed. During the generation of oxidized themoglobin, free radicals are formed [27] that may initiate lipid peroxidation. The formation of lipid peroxides was determined by means of thiobarbituric acid reactive substances (TBARS) [26]. The only oxime that caused detectable TBARS in erythrocytes and hemolysate was acetaldoxime. Therefore, lipid peroxidation is considered as a less sensitive general effect parameter, because only one of the three oximes tested caused lipid peroxidation. However, it does give more specific information about the ability of a compound to initiate lipid peroxidation. Erythrocyte GST activity was lowered after incubation of hemolysate with all three oximes or after incubation of cyclohexanone 
oxime or acetaldoxime with erythrocytes. This can be explained by the fact that free radicals or products of lipid peroxidation can inhibit erythrocyte GST activity $[6,7,23,24]$. The effects found in the incubations with erythrocytes alone were not enlarged when metabolizing systems were added. This indicates that liver metabolism is not of major importance for the hematotoxic effects of oximes.

In conclusion, glutathione depletion is a good parameter when strong electrophilic compounds are tested or when electrophilic metabolites are generated by metabolizing systems. GT depletion can also be used as an effect parameter when strong oxidative compounds are tested, but for this category of compounds there are more sensitive options (e.g. hemoglobin oxidation or GST inhibition). GT depletion is not a sensitive parameter when weak oxidative compounds are tested. In this case, hemoglobin oxidation and GST inhibition showed to be preferable (Table 7.2). As free radicals are generated during the process of $\mathrm{HbFe}^{3+}$ formation caused by hydroxylamine, and oximes may be hydrolyzed into hydroxylamine, the effects of the oximes on GST activity and TBARS formation may be a result of this $\mathrm{HbFe}^{3+}$ formation.

Apart from being a target to reactive metabolites generated and released from tissues, erythrocytes are specific oxygen carriers. Our studies testing the isolated perfused rat liver without oxygen carriers showed degeneration of liver tissue (Chapter 5), especially affecting the perivenous hepatocytes. As the cytochrome P-450 content is high in the perivenous hepatocytes $[9,22,30]$, the usage of medium without oxygen carriers may therefore affect the capacity to oxidize xenobiotics. Also oxygen deprivation that does not lead to degeneration of hepatocytes may lead to a reduced metabolic activity of cytochrome P-450 [2]. However, the isolated liver was still able to metabolize dimethylacetamide (DMAc) which was added to a medium composed of bicarbonate buffered Krebs solution plus $1 \%$ albumin and the elimination velocity constant of DMAc was determined (Chapter 4). DMAc was expected to be metabolised into $\mathrm{N}$-hydroxymethyl $\mathrm{N}$-methylacetamide that may be oxidized further into monomethylacetamide $[4,12,18,25]$. However, monomethylacetamide could not be detected in the perfusates. Since addition of erythrocytes to the medium might influence the capability of the liver to metabolize DMAc, the elimination velocity constant of DMAc was determined in perfusions in which human erythrocytes were added to the perfusion medium (Chapter 5). However, no significant difference was found between the elimination velocity constant of DMAc determined in perfusions with or without human erythrocytes added to the perfusion medium. It was not determined whether human erythrocytes were able to metabolize DMAc. However, if DMAc is metabolised by erythrocytes than the elimination velocity constant in perfusions with erythrocytes would have been higher. It can be concluded that the liver in an isolated perfused liver system metabolizes DMAc and that human erythrocytes have a positive influence on the viability of the liver cells. 
The techniques described in this thesis can be used to evaluate the ability of a compound and/or its metabolites to affect erythrocytes. As it is important to measure the proper targets in erythrocytes, it is recommended to study the chemical properties of a compound and/or its metabolites prior to the choice of the parameters that will be measured. As already mentioned, GT depletion may be a good target when electrophiles have to be detected. In case of weakly oxidative compounds, hemoglobin oxidation and GST inhibition are better parameters to be measured. If an insight into the ability of a compound to affect erythrocyte parameters is wanted, this can be tested in incubations with erythrocytes. Beside a direct effect of the parent compound on the parameter tested also metabolites generated by the erythrocytes may be responsible for the effects found. If $\mathrm{GT}$ depletion is chosen as an effect parameter, incubation of the test compound with GSH solution is an option to circumvent the possibility of metabolite generation by erythrocytes. The ability of metabolites generated by rat liver preparations to affect erythrocyte parameters can be tested in co-incubations of erythrocytes and rat liver derived metabolizing systems. Since metabolites generated by microsomes do not have to pass a hepatocyte membrane and as there is no conjugation with endogenous substrates, a greater effect on erythrocyte parameters is expected to be found in co-incubations of erythrocytes and MAS compared to co-incubations in which hepatocytes or a wholle liver are tested. This was was confirmed in the studies performed in this thesis.

Co-incubations of erythrocytes and hepatocytes may give an insight into the ability of metabolites generated by hepatocytes to leave the hepatocyte, enter the erythrocyte and affect the erythrocyte parameters tested. The metabolites are probably transported over the membranes by diffusion. One of the factors determining the amount of metabolite that passes the erythrocyte membrane by diffusion is the amount of metabolites generated and excreted by the hepatocytes. In this way, the concentration gradient over the erythrocyte membrane determines the intra-ery throcyte metabolite concentration that may give a detectable change in the erythrocyte parameter(s) measured. Beside the concentration gradient over the membrane, also the time of incubation affects the amount of metabolite(s) that passes the erythrocyte membrane. In Chapter 3 , the diffusion equations described by Fick were used to gain insight into the permeability coefficient of the metabolite(s) of 3-hydroxyacetanilide. Glutathione conjugation was the effect parameter tested. Since the reaction between glutathione and the metabolite formed is equimolar, the minimal amount of metabolite that had to be formed per unit of membrane and time so that glutathione depletion could be detected was calculated. Using this value, the permeability constant that must minimally be reached to find glutathione depletion was calculated for the metabolite concentration which was found outside the erythrocytes.

The incubations performed in this thesis can be extended with ghosts to test whether hemoglobin plays an essential role in the generation of TBARS when ery- 
throcytes or hemolysate are incubated with acetaldoxime. Purthermore, ghosts may also be filled with target systems that may be affected by a xenobiotic or its metabolite(s). The incubations with Transwells ${ }^{T M}$ can be improved by increasing the concentration gradient over the erythrocyte membrane. This can be done by increasing the amount of hepatocytes added to the system so that the amount of metabolites generated and excreted by the hepatocytes may increase. The amount of metabolites formed by the hepatocytes may also be increased by pretreatment of rats with enzyme inducers. Since the metabolites generated by the hepatocytes were diluted to a great extent as a consequence of the technical conditions set by the Transwells ${ }^{T^{M} M}$, concentration of the test system by using smaller volumes in which the erythrocytes and hepatocytes are diluted may also help. Another possibility to enlarge the sensitivity of this test system is increasing the time of incubation. In this way, higher amounts of metabolites may be formed and the amount of metabolite that passes the erythrocyte membrane by diffusion increases. However, it may be necessary to use sterile techniques when the incubation period has to be extended. However, incubation periods of more that 24 hours are not expected to be effective as cytochrome P-450 activity of hepatocytes decreases drastically with time [14]. The effects found in human erythrocytes that are perfused through an isolated rat liver may be increased by means of the same variables mentioned for Transwells ${ }^{T M}$.

Based on the results obtained in this thesis it can be conciuded that erythrocyte parameters were affected by several test compounds. Which parameter(s) was/were affected depended on the compound tested. In in vitro incubations with hemolysate the effects were higher than in incubations with erythrocytes. Hemolysate was able to generate a detectable amount of GSH-reactive metabolites out of some test compounds. This was not the case when the same substrates were tested in incubations with erythrocytes. GSH or GT depletion was a good effect parameter when electrophilic or strong oxidative compounds were tested, but not when weak axidative compounds had to be detected. In that case hemoglobin oxidation or changes in GST activity were better options. Since compounds not able to affect erythrocyte parameters might be able to do so after metabolization by rat liwer preparations, co-incubations of erythrocytes and MAS or hepatocytes, and perfusion of erythrocytes through an isolated rat liver were performed. Test substrates which were able to affect erythrocyte parameter(s) after metabolic activation were only able to do so after co-incubation with MAS. No extra effect on erythrocyte parameters caused by hepatocytes or a total liver could be detected after co-incubation of erythrocytes with hepatocytes or after perfusion of erythrocytes through an isolated liver. This may be explained by detoxification of reactive metabolites in the hepatocytes or by limited diffusion of reactive metabolites over the hepatocyte membrane. Although perfusion through an isolated liver of medium enriched with erythrocytes did not increase the effects found in incubations with erythrocytes alone, erythrocytes on their 
turn played an important role in the maintenance of liver cell viability. Since no effects were found in the test systems that approximated the in vivo situation more than MAS, this indicates that the sensitivity of the test systems tested in this thesis decreased with the complexity of the test system. This may be explained by the capacity of hepatocytes and the total liver to adapt to changing circumstances. 


\section{Bibliography}

[1] Akerboom, T. and Sies, H. Glutathione transport and its significance in oxidakive stress, in: Viña, J. (ed.) Glutathione: metabolism and physiological functions, CRC Press, Boca Raton, 1990, 46-55.

[2] Amgus, P.W., Morgan, D.I. and Smallwood R.A. (1990) Review article: hyporia and hepatic drug metabolism-clinical implications. Aliment. Pharmacol. Therap. $4,213-225$.

[3] Belvedere, G. and Tursi, F. (1981) Styrene oxidation to styrene oxide in human blood erythrocytes and Iymphocytes. Res. Commun. Chen. Pathol. Pharmacol. $33,273-282$.

[4] Borm, P.J.A., DeJong, L. and Vliegen, A. (1987) Environmental and biological monitoring workers occupationally exposed to dimethylacetamide. J. of Occup. Med. 29, 898-903.

[5] Duverger-van Bogaert, M., Crutzen-Fayt, M.C. and Stecca, C. (1991) Mutagenicity of some heterocyclic amines in Salmonella typhimurium with metabolic activation by human red blood cell cytosol. Mutat. Res. 261, 261-265.

[6] Evelo, C.T.A., Palmen, N.G.M., Arthur, Y. and Janssen, G.M.E. (1992) Changes in blood glutathone concentrations, and in erythrocyte glutathione reductase and glutathione S-transferase activity after running training and after participation in contests. Eur. J. Appl. Physiol. 64, 354-358.

[7] Evelo, C.T.A., Bisschops, R.A.G., Baars, L.G.M., Spooren, A.A.M.G. and Neis, J.M. Two mechanisms for toxic effects of hydroxylamines in human erythrocytes; Involvement of free radicals and risk of potentiation. Toxicol. Appl. Pharmacol. submitted for publication.

[8] Gebhardt, R. (1992) Metabolic zonation of the liver: regulation and implications for liver function. Pharmac. Ther. 53, 275-354.

[9] Gooding, P.E., Chayen, J., Sawyer, B. and Slater, 'T.F. (1978) Cytochrome P-450 distribution in rat liver and the effect of sodium phenobarbitone adminitration. Chem. Biol. Interact. 20, 299-310.

[10] Grossman, S.J., Simson, J. and Jollow, D.J. (1992) Dapsone-induced hemolytic anemia: effect of N-luydroxy dapsone on the sulfhydryl status and membrane proteins of rat erythrocytes. Toxicol. Appl. Pharmacol. 117, 208-217.

[11] Guyton, A.C. Textbook of medical physiology, W. B. Saunders company, Philadelphia, 1986, pp. 41-50.

[12] Kennedy, G.L and Pruet, J.W. (1989) Biologic monitoring for dimethylacetamide: measurement for 4 consecutive weeks in a workplace. J. Oxcup. Med. 31, 47-50.

[13] Kondo, T., Dale, G.L. and Beutler, E. (1981) Studies on glutathione transport utilizing inside-out vesicles prepared form human erythrocytes. Biochim. Biophys. Acta $645,132-136$.

[14] Kuwahara, S.K. and Mannering G.J. (1993) Effects of interferon, polyriboinosinic acid-polyribocytidilic acid and steroids on the cytochrome P-450 gystem of cultured primary mouse hepatocytes. Pharmacol. Toxicol., $72,199-204$.

[15] López-Barea, J., Bárcena, J.A., Bocanegra, J.A., Florindo, J., García-Alfonso, C., López-Ruiz, A., Martínez-Galisteo, E. and Peinado, "I. Structure, nechanism, functions, and regulatory properties of glutathione reductase, in: Viña, J. (ed.) 
Glutathione: metabolism and physiological functions, CRC Press, Boca Raton, 1990, pp. $105-116$.

[16] Lo Jacono, F., Stecca, C. and Duverger, M. (1992) Mutagenic activation of benzo[a]pyrene by human red blood cells. Mutat. Res. 268, 21-26.

[17] Mannervik, B., Widersten, M. and Board, P.G. Glutathione-linked enzymes in detoxication reactions, in: Taniguchi, N., Higashi, T., Sakamoto, Y. and Meister, A. (eds.) Glutathione centennial: moleculare perspectives and clinical implications, Academic Press, San Diego, 1989, pp. 23-34.

[18] Maxfield, M.E., Barnes, J.R., Azar, A. and Trochimowicz, H.T. (1975) Urinary excretion of metabolite following human exposure to dimethylformamide or dimethylacetamide. J. Occup. Med. 17, 506-511.

[19] Mieyal, J.J., Ackerman, R.S., Blumer, J.L. and Freeman, L.S. (1976) Characterizar tion of enzyme-like activity of human hemoglobin; Properties of the hemoglobin-P. 450 reductase-coupled aniline hydroxylase system. $\mathrm{J}$. Biol. Chem. 251, 3436-3441.

[20] Norppa, H., Vainio, H. and Sorsa, M. (1983) Metabolic activation of styrene by erythrocytes detected as increased sister chromatid exchanges in cultured human lymphocytes. Cancer Res. 43,3579-3582.

[21] Scott, M.D., Zuo, L., Lubin, B.H. and Chiu, D.T.-Y. (1991) NADPH, not glutathione, status modulates oxidant sensitivity in normal and glucose 6-phophate dehydrogenase-deficient erythrocy tes. Blood 77, 2059-2064.

[22] Seibert, B., Oesch, F. and Steinberg, P. (1989) Distribution and induction of cytochrome P-450 and two cytochrome P-450-dependent monooxygenase activities in rat liver parenchymal cell populations separated by centrifugal elutriation. Arch. Toxicol. 63, 18-22.

[23] Shen, H., Tamai, K., Satoh, K., Hatayama, L., Tsuchida, S. and Sato, K. (1991) Modulation of class pi glutathione transferase activity by sulfhydryl group modification. Arch. Biochem. Biophys. 286, 178-182.

[24] Shen, H., Tsuchida, S., Tamai, K. and Sato, K. (1993) Identification of cysteine residnes involved in disulfide formation in the inactivation of elutathione transferase P-form by hydrogen peroxide. Arch. Biochem. Biophys. 300, 137-141.

[25] Sherman, H. and Barnes, J.A. as referenced by Kennedy, G.L. (1986) Biological effects of acetamide, formanide and their monomethyl and dimethyl derivatives. CRC Crit. Rev. Toxicol. 17, 129-182.

[26] Sinnhuber, R.O., Yu, T.C. (1957), 2-Thiobarbituric acid method for the measurement of rancidity in fishery products; II The quantitative determination of malonaldelyde. Food Technol. 12, 9-12.

[27] Stolze, K. and Nohl, H. (1989) Detection of free radicals as intermediates in the methemoglobin formation from oxyhemoglobin induced by hydroxylamine. Biochem. Pharmacol. 38, 3055-3059.

[28] Stryer, L., Biochemistry, W.H. Freeman and company, New York, 1988, pp. 349438.

[29] Tursi, F., Samaia, M., Salmona, M. and Belvedere, G. (1983) Styrene oxidation to styrene oxide in human erythrocytes is catalyzed by oxyhemoglobin. Experientia $39,593-594$ 
[30] Wanatabe, J., Kanai, K. and Kanamura, S. (1989) A new microphotometric method for measurement of cytochrome P-450 in sections of liver. J. Histochem. Cytochem. 37, 1257-1263.

[31] Yubisui, T., Matsuki, T., Tanishima, K., Takeshita, M. and Yoneyama, Y. (1977) NADPH-flavin reductase in human erythrocytes and the reduction of methemoglobin through flavin by the enzyme. Biochem. Biophys. Res. Comm. 76, 174182.

[32] Yubisui, T., Takeshita, M. and Yoneyama, Y. (1980) Reduction of methemoglobin through flavin at the physiological concentration by NADPH-flavin reductase of human erythrocytes. J. Biochem. 87, 1715-1720. 



\section{Chapter 8}

\section{Summary}

Blood plays an important role in the body because of its transport function. The transport of oxygen to the tissues and of carbondioxyde to the lungs is confined to erythrocytes. Because of their central role, erythrocytes come in close contact with organs that may absorb foreign compounds (xenobiotics) and with organs that may transform non-toxic compounds into reactive metabolites. Erythrocytes also have metabolic activity themselves. The cells contain several mechanisms of protection, usually of biochemical origin, against toxic compounds or their metabolites. Cell damage may occur when the balance between the stress erythrocytes are exposed to and the capacity of detoxification is disturbed.

In the liver erythrocytes come in close contact with the metabolically very active hepatocytes. Non-toxic compounds may be metabolised by hepatocytes into toxic metabolites that may affect the erythrocytes. In this thesis main attention is directed to the interplay between erythrocytes and hepatocytes with special emphasis to the bioactivation and inactivation capacity of liver and blood.

Glutathione plays a role in the protection of erythrocytes against electrophilic and oxidative stress. Chapters 2 and 3 show that strong electrophilic compounds that are able to pass the erythrocyte membrane (iodoacetamide, $N$-ethylmaleimide and diethyl maleate) were able to deplete the concentration of reduced glutathione (GSH) in erythrocytes. Since the test compounds also depleted GSH in incubations with GSH dissolved in buffer (GSH solution), this means that they are able to deplete GSH without prior metabolic activation. From the experiments with hemolysate we conclude that 3-hydroxyacetanilide (3-HAA) and 2-methylfurane (2-MF) are metabolized by hemolysate into GSH-reactive compounds. The total glutathione concentration ( $(G T)$ which is the sum of both GSH and oxidized glutathione) in hemolysate decreased with the concentration of the test compound while the GT concentration was not affected in incubations with GSH solution. Both 3-HAA and 2-MF did not affect erythrocyte GT which may be explained by a low intracellular substrate concentration. Since hemolysate is not the same as erythrocytes without a cellular membrane, the difference in effect: between incubations with erythrocytes and hemolysate cannot be explained only by these 
cellular membranes. Compounds that cannot deplete erythrocyte GT may be able to do so after extracellular metabolic activation. Both cyclophosphamide (CP), 3-HAA and 2-MF were able to deplete erythrocyte GT after metabolic activation by means of rat liver microsomal activating system (MAS) indicating that the metabolites were able to cross the erythrocyte membrane. The GT depletion in erythrocytes was higher when microsomes of rats that were pretreated with phenobarbitone were tested. This means that phenobarbitone inducable enzymes play a role in the metabolization of these test compounds. Two pesticides that are able to form hemoglobin adducts in wivo were not able to affect the GT concentration in incubations with GSH solution, hemolysate or erythrocytes irrespective of the addition of MAS to the incubations. Hepatocytes imitate the in wivo situation more closely than MAS. For this reason, co-incubations of erythrocytes and hepatocytes were performed. Hepatocytes were able to metabolize CP and 3-HAA into GSH-reactive metabolites. Moreover, GSH-reactive metabolites of $\mathrm{CP}$ and $3-\mathrm{HAA}$ were also excreted by the hepatocytes. However, erythrocyte GT was not affected in co-incubations of erythrocytes and hepatocytes testing 3-HAA, which may be explained by a minor diffusion uptake rate of metabolites into the erythrocytes.

In chapters 4 and 5 the in vivo situation was approached more closelly by perfusion of an isolated rat liver with medium and substrate. Liver cell viability was affected when no oxygen carriers were added to the medium. However, despite the lack of oxygen carriers in the medium, dimethylacetamide (DMAc) was eliminated from the medium (elimination rate constant $=0.019 \pm 0.008 \mathrm{~min}^{-1}$ ). The addition of human erythrocytes to the perfusion medium had a positive effect on liver cell viability, which might lead to an increase of DMAc metabolism. However, no significant increase in the elimination rate constant of DMAC was detected.

The techniques which were developed in the preceding experimental chapters were applied to hydroxylamine (HYAM) and three oximes (cyclohexanone oxime (CHO), acetaldoxime (AAO) and methyl-ethyl ketoxime (MEKO)) in chapter 6. HYAM is a strong oxidative compound and oxidizes hemoglobin, causes the formation of thiobarbituric acid reactive substances (TBARS), inactivates glutathione S-transferase (GST) and lowers the GT concentration in erythrocytes. Oximes were less reactive compared to HYAM both in incubations with erythro cytes and hemolysate. In incubations with hemolysate, all three oximes caused oxidation of hemoglobin and inactivation of GST. These effects were also found in incubations with erythrocytes with exception of inactivation of GST caused by MEKO. However, the effects were less pronounced which may be explained by a low intracellular substrate concentration. AAO was the only oxime that caused TBARS both in incubations with erythrocytes and hemolysate. GT depletion was the least sensitive parameter tested since only MEKO was able to deplete GT in a concentration dependent way and this was only true for experiments with 
hemolysate. Because oximes cause oxidative effects in blood in vivo and oximes are suggested to be metabolized into HYAM, rat liver preparations were added to the incubations with erythrocytes. However, the effects found in erythrocytes after co-incubation of erythrocytes and microsomal activating system (MAS) or hepatocytes were not higher compared to incubations with erythrocytes alone. This was also true when erythrocytes and oximes were perfused through an isolated rat liver. It is concluded that liver metabolism is not of major importance for the hematotoxic effects of oximes.

Based on the results obtained in this thesis it can be concluded that erythrocyte parameters were affected by several compounds. Which biochemical parameter(s) was/were affected depended on the compound tested. The effects found were higher in incubations with hemolysate compared to incubations with erythrocytes. Hemolysate had metabolic activity since GSH-reactive metabolites were formed out of several test substrates. The same substrates did not affect the GT concentration in erythrocytes. GSH or GT depletion was a proper effect parameter when electrophilic or strong oxidative compounds had to be detected. Hemoglobin oxidation or GST activity clianges were better parameters in case of weak oxidative compounds. Some compounds had to be metabolized by MASS before they affected erythrocyte parameters. Co-incubation of erythrocytes with liver preparations that matched the in vivo situation more closely than MAS (hepatocytes or a total liver), did not enlarge the effects found in erythrocytes compared to incubations with erythrocytes alone. This can be explained by detoxification of reactive metabolites in the hepatocyte and by limited excretion of reactive metabolites from the hepatocyte. Although perfusion of erythrocytes through a isolated liver did not increase the effects found in incubations with erythrocytes alone, erythrocytes on their turn played an important role in the maintenance of liver cell viability. In the incubations in which erythrocytes were co-incubated with liver tissue, only MAS affected erythrocyte parameters. Because of this it can be said that the sensitivity of the test systems decreased when the in wivo situation was approached more closely. This may be explained by the capacity of hepatocytes and the total liver to adapt to changing circumstances. 



\section{Hoofdstuk 9}

\section{Samenvatting}

Bloed speelt een centrale rol in het lichaam vanwege zijn transportfunktie. Daarbij verzorgen de erytrocyten het vervoer van zuurstof naar de weefsels en van kooldioxide terug naar de longen. Als gevolg van hun centrale rol komen erytrocyten in nauw kontakt met zowel organen die kumnen dienen als porte d'entrée voor lichaamsvreemde stoffen (xenobiotica) als met organen die deze xenobiotica kunnen transformeren. Bovendien is de erytrocyt zelf in staat metabolieten te genereren. Om zich tegen toxische verbindingen of hun metabolieten te beschermen bevat de erytrocyt diverse beschermingsmechanismen die vaak zijn gebaseerd op biochemische reakties. Wanneer de stress waaraan een cel wordt blootgesteld zo groot, is dat de toxische verbinding niet wordt gedetoxificeerd kan dat leiden tot schade aan de cel.

In de lever komen erytrocyten in nauw kontakt met de metabool zeer aktieve hepatocyten. Niet toxische stoffen kunnen na metabolisering door de lever worden omgevormd in stoffen die toxisch zijn voor de erytrocyten. In dit proefschrift wordt voornamelijk aandacht besteed aan het samenspel tussen erytrocyten en hepatocyten waarbij met name aandacht wordt besteed aan de bioaktiverende en inaktiverende kapaciteit van zowel het bloed als de lever. Om deze vraagstelling te kunnen onderzoeken werden een aantal in vitro methodieken ontwikkeld.

Glutathion speelt een rol bij de bescherming van erytrocyten tegen elektrofiele en oxidatieve stress. Uit de hoofdstukken 2 en 3 kunnen we afleiden dat de koncentratie gereduceerd glutathion (GSH) in erytrocyten daalde wanneer de cellen werden blootgesteld aan sterk elektrofiele verbindingen (joodacetamide, $\mathbb{N}$ ethylmaleimide en diethyl maleaat) die in staat zijn de erytrocyt mernbraan te passeren. Genoemde verbindingen waren ook in staat GSH te depleteren in inkubaties met GSH opgelost in buffer (GSH oplossing), hetgeen betekent dat ze in staat zijn GSH te depleteren zonder voorafgaande metabolisering. Vit de experimenten met hemolysaat kunnen we afleiden dat 3-hydroxyacetanilide (3-HAA) en 2-methylfuraan (2-MF) door het hemolysaat worden gemetaboliseerd tot GSHreaktieve verbindingen. De koncentratie totaal glutathion ((GT) dit is de som van de GSH en de geoxideerde glutathion (GSSG) koncentratie) in het hemo- 
lysaat daalde koncentratieafhankelijk terwijl de GT koncentratie in inkubaties met GSH oplossing niet werd beïnvloed. 3-HAA en 2-MF hadden geen effekt op het GT gehalte in erytrocyten, mogelijk als gevolg van een te lage intracellulaire substraat koncentratie. Omdat hemolysaat niet gelijk mag worden gesteld aan erytrocyten zonder celmembraan, mag het verschil in effekt tussen inkubaties met hemolysaat en erytrocyten niet alleen worden verklaard uit de afwezigheid van de celmembraan.

Stoffen die niet in staat zijn GT in erytrocyten te depleteren kunnen dit mogelijk wel na extracellulaire metabole aktivering. Zowel cyclophosphamide (CP) als 3HAA als 2-MF waren in staat de GT koncentratie in erytrocyten te depleteren na metabole aktivering door middel van microsomaal aktiverend systeem (MAS). De GT depletie werd nog versterkt wanneer in de inkubaties gebruik werd gemaakt van MAS van ratten die waren voorbehandeld met fenobarbital. Dit wijst op de betrokkenheid van fenobarbital induceerbare enzymen bij het metabolisme van bovenstaande verbindingen. Omdat de metabolieten in staat waren de GT koncentratie in erytrocyten te depleteren, mag hieruit worden afgeleid dat ze de celmembraan kunnen passeren. Twee pesticiden waarvan bekend is dat ze in vivo hemoglobine addukten vormen hadden geen effekt op de GT koncentratie in inkubaties met GSH oplossing, hemolysaat of erytrocyten. Toevoeging van MAS aan de inkubaten had geen effelkt. Hepatocyten benaderen de in vivo situatie meer dan MAS. Om deze reden werden tevens co-inkubaties van erytrocyten met hepatocyten uitgevoerd. Hepatocyten waren in staat GSH-reaktieve verbindingen te genereren uit CP en 3-HAA. Tevens werden er GSH-reaktieve CP en 3-HAA metabolieten door de hepatocyt uitgescheiden. In co-inkubaties van erytrocyten en hepatocyten met 3-HAA als teststof kon echter geen GT depletie in erytrocyten worden waargenomen, hetgeen mogelijk verklaard kan worden door een te geringe opnamesnelheid van de metabolieten in de erytrocyt.

In de hoofdstukken 4 en 5 werd de in vivo situatie nog meer benaderd door een totale geissoleerde lever te perfunderen met medium waaraan de teststof is toegevoegd. Perfusiemedium zonder zuurstofcarriers bleek de levensvatbaarheid van de levercellen ernstig aan te tasten. Dimethylacetamide (DMAc) werd ondanks het gebrek aan zuurstofcarriers in het perfusiemedium toch geëlimineerd met een eliminatiesnelheidskonstante van $0,019 \pm 0,008 \mathrm{~min}^{-1}$. Toevoeging van humane erytrocyten aan het medium had een positieve invloed op de levensvatbaarheid van de levercellen, hetgeen tot een verhoogde biotransformatie van DMAc zou kunnen leiden. Een signifikante verhoging van de eliminatiesnelheidskonstante van DMAc kon echter niet worden aangetoond.

In hoofdstuk 6 werden de technieken die in de voorgaande experimentele hoofdstukken werden gebruikt toegepast op hydroxylamine (HYAM) en een drietal oximen (cyclohexanonoxime (CHO), acetaldoxime (AAO) en methyl-ethyl ketoxime (MEKO)). HYAM staat bekend als een sterk oxidatieve verbinding en 
oxideert hemoglobine, induceert lipide peroxidatie (LP), inaktiveert glutathion S-transferase (GST) en verlaagt de GT koncentratie in erytrocyten. De oximes bleken veel minder reaktief te zijn dan HYAM zowel in inkubaties met erytrocyten als met hemolysaat. In inkubaties met hemolysaat veroorzaakten alle geteste oximen oxidatie van hemoglobine en inaktivering van GST. De effekten waren, met uitzondering van GST inaktivering door MEKO, ook zichtbaar in erytrocyten hoewel ze minder sterk waren. Dit kan het gevolg zijn van een te lage intracel lulaire substraat koncentratie. Alleen AAO veroorzaakte LP, zowel in inkubaties met erytrocyten als met hemolysaat. GT depletie bleek de minst gevoulige parameter te zijn ondat alleen MEKO een duidelijke koncentratieafhankelijke GT depletie veroorzaakte, en dan nog alleen in inkubaties met hemolysaat. Ondat oximes in wivo oxidatieve effekten in bloed veroorzaken en ondat oximes mogelijk worden gemetaboliseerd tot HYAM, werden rattelever preparaten toegevoegd aan de inkubaties met erytrocyten. De grootte van de effekten die in inkubaties met alleen erytrocyten konden worden angetoond, nam echter niet toe na coinkubatie van erytrocyten met MAS of hepatocyten. Hetzelfde gold voor perfusie van een geisoleerde rattelever met medium waaraan oximen en erytrocyten waren toegevoegd. Op basis wan deze resultaten kan worden gesteld dat metabole omzetting van oximen door de lever geen belangrijke rol speelt in de hematotoxische effekten van oximen.

Op basis van de resultaten verkregen in dit proefschrift kan worden gesteld dat verschillende xenobiotica in staat zijn biochemische parameters in erytrocyten te beinvloeden. Welke biochemische parameters veranderden als gevolg van expositie aan xenobiotica, was afhankelijk van de geteste stof. Vergeleken bij inkubaties met erytrocyten waren de effecten in inkubaties met hemolysaat groter. Hemolysaat beschikt tevens over metabole kapaciteit omdat een antal substraten werden gemetaboliseerd tot GSH-reaktieve verbindingen. Dezelfde substraten hadden geen effekt op de GT koncentratie in erytrocyten. GSH en GT depletie waren goede effekt parameters wanneer het elektrofiele of sterk oxidatieve verbindingen betrof. Zwak oxidatieve verbindingen konden beter worden aangetoond door middel van hemoglobine oxidatie of alktiviteitsverandering van GST. Sommige verbindingen beinvloedden de erytrocyt parameters pas na metabole aktivering met behulp van MAS. Gebruik van aktiverende leverpreparaten die de iw viwo situatie beter benaderden dan MAS (hepatocyten of de geisoleerde rattelever), vergrootten de effekten die werden gevonden in inkubaten met alleen erytrocyten niet. Dit. kan worden verklaard door ontgifting van reaktieve metabolieten in de hepatocyt en door een geringe uitscheiding door de hepatocyt van reaktieve metabolieten. Ofschoon perfusie van erytrocyten door een geisoleerde lever geen effekt had op de gemeten parameters in de erytrocyten, hadden erytrocyten wel een positieve invloed op de levensvatbarheid van de levercellen. Omdat van alle testsystemen waarin gebruik werd gemaakt van co-inkubaties van erytrocyten met leverweefsel alleen MAS effekt had op de gemeten parameters in de erytrocyten, kan worden 
gesteld dat de gevoeligheid van de testsystemen afneemt naarmate ze de in vivo situatie beter benaderen. Dit kan mogelijk verklaard worden door het vermogen tot adaptatie wan hepatocyten en de totale lever. 


\section{Dankwoord}

Een proefschrift is nooit het produkt van én persoon. Ook bij mij was dat zeker niet het geval. Het promotieonderzoek werd gestart binnen de voormalige vakgroep Arbeidsgeneeskunde, Milieugezondheidkunde en Toxicologie (AMT) en, nadat deze vakgroep werd opgeheven, binnen de vakgroep Farmacologie afgerond. $\mathrm{Bij}$ deze wil ik jullie, de medewerkers van beide valkgroepen, bedanken voor jullie collegialiteit. Door jullie heb ik, ondanks alle tegenslagen, toch een fije tijd gehad.

Ondat het een sterk laboratoriumgebonden onderzoek betrof en ondat gedurende het grootste cleel van de promotieperiode een analist op het laboratorium ontbrak, was ik aangewezen op de hulp van studenten en stagiaires van de studie Gezondheidswetenschappen te Maastricht, de Hogere Laboratoriumschool te Eindhoven en de Middelbare Laboratoriumschool te Sittard. Ondat het aantal mensen dat stage heeft gelopen binnen mijn projekt erg groot is, en omdat ik niemand wil vergeten, noem ik geen namen maar wil ik bij deze iedereen heel hartelijk danken voor zijn inzet.

Naast hulp bij het uitwoeren van de experimenten is wetenschappelijke backup van essentieel belang tijdens het leerproces dat een AlO doorloopt. Hierin heeft Chris Evelo de meest prominente rol gespeeld. Chris, bij deze wil ik je heel hartelijk danken voor je inzet en de fijne wijze waarop je dat hebt gedaan. Ik hoop dat we ook in de toekomst nog regelmatig met elkar zullen samenwerken. Tevens wil ik Pie Henderson, Paul Borm en Harry Strujiker Boudier bedanken voor hum aandeel in het onderzoek. Jullie suggesties waren zeer welkom. Anita, IJmert en Hans, de discussies die ik met jullie heb gevoerd waren voor mij heel waardevol. Tevens wil ik een woord van dank richten aan Leo.

Mijn ouders hebben mij altijd de gelegenheid gegeven om te studeren en daar blijf ik ze altijd dankbaar voor. Daniëlle, de vele uren dat we samen hebben gestudeerd en gediscussieerd waren voor mij heel waardevol. Tot slot wil ik mijn vrienden bedanken voor hun steun en begrip met name in de afrondingstase var het onderzoek. 



\section{Curriculum vitae}

Nicole Palmen werd geboren op 23 april 1964 te Susteren. In 1982 behaalde zij het diploma Atheneum-B aan het Bisschoppelijk College te Echt. Daarna studeerde zij Gezondheidswetenschappen aan de Rijksuniversiteit Limburg te Maastricht en specialiseerde zich in de afstudeerrichtingen Verplegingswetenschap en Biologische Gezondheidkunde, die zij afsloot met het behalen van de Doctoraaldiploma's in respectievelijk 1987 en 1988.

Van augustus 1988 tot angustus 1993 was zij als Assistent in Opleiding in dienst van de Rijksuniversiteit Limburg. Het onderzoek werd gestart bij de vakgroep Arbeidsgeneeskunde Milieugezondheidkunde en Toxicologie (hoofd: prof. dr. P.Th. Henderson), en vervolgd bij de vakgroep Farmacologie (hoofd: prof. dr. H.A.J. Struijker Boudier), sectie Toxicologie. De dagelijkse begeleiding was in handen van dr. ir. C.T.A. Evelo. Tijdens de promotieperiode volgde ze tevens een aantal cursussen in het kader van de post-doctorale opleiding Toxicologie.

Sinds maart 1994 is zij aangesteld als arbeidstoxicoloog en arbeidshygiënist bij de Arbodienst Limburg en volgt momenteel de cursus arbeidshygiëne aan de Hogeschool West-Brabant. 


\section{Lijst van Publicaties}

\section{Full papers:}

Borm, P.J.A., Palmen, N.G.M., Engelen, J.J. and Buurman, W.A. (1988) Spontaneous and stimulated release of tumor necrosis factor-a (TNF) from blood monocytes of miners with coal worker's pneumoconiosis. Am. Rev. Resp. Dis. $138,1589-1594$.

Palmen, N.G.M., Evelo, C.T.A., Borm, P.J.A. and Henderson, P.Th. (1992) Infuence of oxygen supply on liver condition and elimination of dimethylacetamide in the isolated perfused rat liver. Toxicol. Vitro 6, 357-365.

Evelo, C.T.A., Palmen, N.G.M., Artur, Y. and Janssen, G.M.E. (1992) Changes in blood glutathione concentrations, and in erythrocyte glutathione reductase and glutathione S-transferase activity after running training and after participation to contests. Eur. J. Appl. Physiol. 64, 354-358.

Palmen, N.G.M., Evelo, C.T.A., Borm, P.J.A. and Henderson, P.Th. (1993) Toxicokinetics of dimethylacetamide (DMAc) in rat isolated perfused liver. Hum. Exp. Toxicol. 12, 127-133.

Palmen, N.G.M. and Evelo, C.T.A. (1993) Glutathione depletion in human erythrocytes as indicator for microsomal activation of cyclophosphamide and 3hydroxyacetanilide. Toxicology 84, 157-170.

Palmen, N.G.M. and Evelo, C.T.A. Glutathione depletion in human erythrocytes and rat liver, a study on the interplay between bio-activation and inactivation functions of liver and blood. Submitted for publication.

Palmen, N.G.M., Feijts, A.H.J.M.G. and Evelo, C.T.A. Oxidative effects in human erythrocytes caused by some oximes and hydroxylamine. Submitted for publication.

\section{Report:}

Evelo, C.T.A., Palmen, N.G.M., Borm, P.J.A., Schins, P.F.R., Zondag, R, Pisters, R. and Leummens, M. (1991) Fysiologisch gebaseerd farmacokinetiek model (PB-PK model) voor 1,3-butadieen. Rapport opgesteld in opdracht van DSMLimburg bv, kunststoffendivisie (KDS), Geleen. 


\section{Abstracts}

Palmen, N.G.M., Sesink, A.I.A., Henderson, P.Th. and Borm, P.J.A. (1989) Application of recirculating and single pass liver perfusions to study interactions between workplace agents and drugs. Pharmaceutisch Weekblad Sci. Ed. 11, suppl. J, J8.

Evelo, C.T.A., Palmen, N.G.M., Bell, O.H.H., Kant, Y. and Borm, P.J.A. (1990) Physiological simulation model for study of metabolic interactions: choice of simulation package. Pharmaceutisch Weekblad Sci. Ed. 12, suppl. B, B6.

Evelo C.T.A., Palmen, N.G.M., Bell, O.H.H., Kant, Y. and Borm, P.J.A. (1990) Physiologically simulation model performance and usability when integrated in different simultation packages. International workshop on pharmacokinetic modelling in occupational health. Leysin Zwitserland, march 4-8.

Palmen, N.G.M., Borm, P.J.A., Sesink, A.L.A. and Henderson, P.Th. (1990) Application of recirculating and single pass lever perfusion to study effects of combined exposure. Leysin Zwitserland, march 4-8.

Palmen, N.G.M., Borm, P.J.A., Sesink, A.I.A., Evelo, C.T.A. and Henderson, P.Th. (1990) Liver perfusion and physiological simulation to study the effect of combined exposure. Beltox second annual meeting. Leuven, march 31, p. 7.

Palmen, N.G.M., Evelo, C.T.A. and Henderson, P.Th. (1991) Depletion of erythrocyte glutathione by extracellularly activated metabolites. Third international ISSX meeting, Amsterdam, june 24-28, Abstract no. 5A24.

Evelo, C.T.A., Palmen, N.G.M., ten Berge, W.F. and Borm, P.J.A. (1991) Physiologically based pharmacokinetic modelling of 1,3 butadiene: increased importance of lung metabolism at low doses. Third international ISSX meeting, Amsterdam, june 24-28, Abstract no. 9A10.

Palmen, N.G.M., Evelo, C.T.A., Borm, P.J.A. and Henderson, P.Th. (1991) Saturation of DMAc metabolism in isolated perfused rat liver. The 1991 Eurotox congress, Maastricht, september 1-4, Abstract no. 153.

Palmen, N.G.M., Evelo, C.T.A. and Henderson, P.Th. (1991) Depletion of erythrocyte glutathione by metabolites formed by fortified liver microsomes. The 1991 Eurotox congress, Maastricht, september 1-4, Abstract no. 154.

Evelo, C.T.A., Palmen, N.G.M., Pisters, R. and Borm, P.J.A. (1991) Development of a new software package for physiologically based pharracokinetic modelling. The 1991 Eurotox congress, Maastricht, september 1-4, Abstract no. 243.

Evelo, C.T.A., Palmen, N.G.M., ten Berge, W.F. and Borm, P.J.A. (1991) Physiologically based kinetic modelling of 1,3 butadiene: importance of lung metabolism increased at low exposures. The 1991 Eurotox congress, Maastricht, september 1-4, Abstract no. 244. 
Paimen, N.G.M., Evelo, C.T.A. and Henderson, P.Th. (1993) Glutathione depletion as an indicator of reactive metabolite formation in microsomes. Hum. Exp. Toxicol. 12, 71-72.

Spooren, A., Palmen, N.G.M. and Evelo, C.T.A. (1993) Influences of cyclophosphamide on the glutathione S-transferase activity and glutathione concentration in rat liver fractions and human erythrocytes. Hum. Exp. Toxicol. 12, 78.

Palmen, N.G.M. and Evelo, C.T.A. (1994) Hydroxylamine and some oximes cause oxidative effects in human erythrocytes. Hum. Exp. Toxicol. 13, 729. 